\title{
Color Perceptions of Deuteranopic and Protanopic Observers
}

\author{
By Deane B. Judd
}

\begin{abstract}
It is well established that about 2 percent of otherwise normal human males are confusers of red and green from birth. There is considerable interest in the question: What do red-green confusers see? From a knowledge of the normal color perceptions corresponding to deuteranopic and protanopic red and green, we may not only understand better why color-blindness tests sometimes fail, and so be in a position to develop improved tests, but also the color-deficient observer may understand better the nature of his color-confusions and be aided to avoid their consequences. If an observer has trichromatic vision over a portion of his total retinal area, and dichromatic vision over another portion, he may give valid testimony regarding the color perceptions characteristic of the particular form of dichromatic vision possessed by him. Preeminent among such observers are those born with one normal eye and one dichromatic eye. A review of the rather considerable literature on this subject shows that the color perceptions of both protanopic and deuteranopic observers are confined to two hues, yellow and blue, closely like those perceived under usual conditions in the spectrum at 575 and $470 \mathrm{~m} \mu$, respectively, by normal observers. By combining this result with standard response functions recently derived (Bureau Research Paper RP1618) for protanopic and deuteranopic vision, it has been possible to give quantitative estimates of the color perceptions typical of these observers for the whole range of colors in the Munsell Book of Color. These estimates take the form of protanopic and deuteranopic Munsell notations, and by using them it is possible not only to arrange the Munsell papers in ways that presumably appear orderly to red-green confusing dichromats, but also to get immediately from the notations an accurate idea of the colors usually perceived in these arrangements by deuteranopes and protanopes, much as the ordinary Munsell notations serve to describe the visual color perceptions of a normal observer.
\end{abstract}

\section{Introduction}

The question, "What colors do color-blind observers confuse?" is a very practical one, capable of objective solution by putting each colorblind observer to trial and noting his mistakes. For the two most common forms of partially color-blind observers, deuteranopia and protannopia, each form comprising about 1 percent of the otherwise normal male population, a general answer, approximately valid for all observers properly so classed, has been found and described $[51,81] .^{1}$

The question, "What colors do color-blind observers see?" is a more subtle one, involving a fine point in the theory of knowledge, yet of considerable practical importance, too. Strictly

${ }^{1}$ Figures in brackets indicate the literature references at the end of this paper.

speaking, the subjective life of each observer cannot be known to anyone else, and there is no way of knowing whether the perception of red by one normal observer bears any resemblance to that which another normal observer calls by the same name. In this sense it is also impossible to discover what colors are seen by the partially color-blind. However, from the similarity in responses between normal observers, it is a fairly safe conclusion that their color perceptions are closely similar. In the same inductive sense, it is possible to discover how the color perceptions of the color-blind are related to those of the normal observer. It is one purpose of the present paper to review the evidence by which these are known. A second purpose is to give a comprehensive intertranslation between normal color perceptions of surface colors on the one hand and 
protanopic and deuteranopic surface-color perceptions on the other.

The outstanding characteristic of protanopic and deuteranopic observers is that they confuse both red and green with gray, and indeed they confuse red with green. For this reason they are rightly called red-green confusers, less aptly red-green blind. Since to the normal eye red is nearly as distinct from green as black is from white, it may be deduced that red-green confusers fail to experience both the normal red and the normal green perception; but the deduction may not be pushed further. From a stimulus normally yielding red, a red-green confuser might perceive red or green or some other of the normal color perceptions, or some perception not normally experienced.

The impossibility of passing from knowledge of what colors are confused to knowledge of those that are seen by color-blind observers was recognized almost as soon as color-blindness was discovered. Thus we find Wilson [97] remarking in 1855, "It must be remembered, however, that there is no common language between the colourblind and the colour-seeing." Then in 1880-81 Holmgren gave essentially the argument of the preceding two paragraphs in complete and clear detail and published it in four languages [39, 40, 42, 46]. Donders [15] in 1881 stated (p. 84), "If they (the color-blind) name their colors yellow and blue, this by no means proves that they see yellow and blue as we see them, but merely that in our yellow and blue their colors appear most characteristically." A year later v. Kries inserted the argument into his early analysis of visual sensations [60], "For bilateral color-blindness the facts are essentially different. Here is lacking the possibility of comparing directly normal and abnormal sensations with each other. The old statement that we cannot know what the color-blind really sense, self-explanatory as it is, appears, however, still not always to have been fully and completely understood."

These repeated and clear explanations did not, however, prevent Edridge-Green [19] in 1911 from giving a "proof" that red-green confusers see red and violet by means of precisely the same arguments previously used by Pole [82] to "prove" from observations with his own color-blind eyes that they see blue and yellow. These arguments are invalid in either case, as pointed out explicitly by Hartridge [27], but Pole's conjecture, for that is all it was, was corroborated by later valid evidence. After having convinced himself (on insufficient ground) that his sensations were the same as what normal observers call yellow and blue, Pole put forward in 1856 an original, very practical suggestion (Roy. Soc., p. 176 ; Phil. Mag., p. 285) now in widespread daily use, namely, "It has been thought that the use of these colors (red and green) for railway and ship signals becomes dangerous where color-blind persons have to observe them. This danger may be obviated by very simple means - if the green be made a blue green at the same time that the red is a yellow red, they become quite as distinct to the color-blind as to the normal eyed." Present color specifications for marine and railway signaling pair a red slightly on the yellow side with a nonyellowish green, and this choice is also prevalent for stop and go signals for highway traffic. This plan has also been extended only recently by the American Standards Association [52] to the marking of industrial hazards.

The practical importance of inquiring into the color perceptions, in addition to the color confusions, of partially color-blind observers lies in the fact that resolution of this question facilitates consideration of precautions against the dangers of color-blindness and also aids in the design of tests for its detection. Thus Pole made his useful suggestion only after he had made a fortunate conjecture as to the connection between his color vision and those of the normal eyed. By this assumption he supplied himself with a terminology that immediately suggested the kind of red and green safe for railway and ship signals. It is the third purpose of this paper to develop a method of expressing the color-perceptions of the average red-green confuser in terms that are immediately comprehensible both to those trained in the interpretation of colorimetric coordinate systems and to the untrained alike. It is expected that this method will assist both normal observers and redgreen-confusing observers to understand the relation between their two systems and so aid in avoiding the undesirable consequences of redgreen confusion.

\section{Review of the Literature}

It has been pointed out by Holmgren [42], by $\mathrm{v}$. Kries [62], and doubtless by many others, that 
persons born with one normal eye and one colorblind eye give us our most direct evidence of the color perceptions of the color-blind. Less reliable evidence may be obtained from persons who confuse red and green because of diseases of the eyes or optic nerves, and who are therefore familiar with normal colors through past experience before becoming afflicted. And, finally, some information may be obtained from the peripheral parts of the normal retina that respond with confusions similar to those of deuteranopia. Any observer, a portion of whose retinas yields normal color vision while another portion yields either the kind of red-green confusion characteristic of protanopia or that characteristic of deuteranopia, can give valid testimony.

It is now a fairly well accepted view that redgreen confusers see neutral colors (black, gray, white, silver, "colorless" and so on) normally, and chromatic colors of two hues, approximately what normal observers call pure yellow and pure blue [14], with little or no admixture of red and green. Evidence obtained from all three types of admissible observers consistently supports this view. We shall, however, review the literature to see whether this rough indication can be made more precise.

\section{Peripheral Parts of the Normal Retina}

It has been widely stated in textbooks for many years that there is, in the normal eye, a retinal zone of considerable extent (perhaps $20^{\circ}$ to $50^{\circ}$ from the fovea centralis) within which yellow, blue, black, and white are perceived much as at the fovea, but red-green distinctions are scarcely, if at all, possible. Since the luminosity function of this region in a light-adapted state is substantially the same as normal $[30,61,68$ (p. 46)], the properties of this retinal zone near the periphery approach those of deuteranopia, the distinctions from deuteranopia being reduced visual acuity, reduced ability to distinguish both black from white, and yellow from blue, and presence of a slight, instead of vanishing, ability to distinguish red from green. As a preliminary observation will immediately show, the precise hues of the yellowish and bluish colors seen by means of this retinal zone are hard to determine. A spectrum stimulus yielding a color of orange hue by foveal vision yields a progression of less and less reddish hues as it is moved more toward the periphery.
There are determinable for each observer, however, within certain limits the wavelengths of the spectrum stimuli yielding colors of invariable hue regardless of retinal region stimulated. It is obvious that the hues of the bluish and yellowish colors perceived by the nearly deuteranopic peripheral region are to be found among these invariable hues. Table 1 is extracted from a summary by Tschermak [95] and shows results of four investigators. As might be expected from the difficult nature of the observations and the many factors influencing the results $[17 ; 69$, p. 273], there is considerable difference between the reports of the various investigators. Goldmann's results refer to a protanomalous observer [24] and on this account have less weight than the others. Some observers can make such observations with good reliability (Hess, 2 to $3 \mathrm{~m} \mu$ uncertainty), others less certainly (Dreher, 10 $\mathrm{m} \mu$ uncertainty); and there is furthermore a large individual difference (40-m $\mu$ spread among Dreher's three observers). Although Hess' results refer to one observer only (himself), they are probably as good as any, and have, at least, been the most widely quoted [30]. These results indicate the deuteranopic yellow to be a hue corresponding, for normal vision under visual conditions, to about $575 \mathrm{~m} \mu$; and deuteranopic blue to be that corresponding to about $470 \mathrm{~m} \mu$, with uncertainties of about $10 \mathrm{~m} \mu$.

TABLE 1. Stimuli that yield bluish or yellowish colors of invariable hue, regardless of retinal region affected

\begin{tabular}{|c|c|c|c|}
\hline \multirow[t]{2}{*}{ Author } & \multirow[t]{2}{*}{ Year } & \multicolumn{2}{|c|}{$\begin{array}{l}\text { Wavelength of spec- } \\
\text { trum stimulus yield- } \\
\text { ing invariable hue, } \\
\text { regardless of retinal } \\
\text { region }\end{array}$} \\
\hline & & Yellowish & Bluish \\
\hline Hess [33] ... & 1889 & $\begin{array}{l}m \mu \\
574.5\end{array}$ & $m_{\mu}$ \\
\hline Baird [4] & 1905 & 570 & 460 \\
\hline Dreher [17] $\ldots . . .$. & 1912 & 568 & 461 \\
\hline Goldmann [25] $\ldots .$. & 1925 & 567 & 466 \\
\hline
\end{tabular}

\section{Red-Green Confusion Acquired Through Disease}

Toxic agents and disease affecting the conducting (transmissive) elements of the optical apparatus (nerve elements and connections, optic nerve, and tract) cause a progressive lessening of the ability to distinguish red from green. This 
defect becomes progressively worse until red-green blindness is reached, which is distinguished from inherited deuteranopia chiefly by its poorer lightdark discrimination. There is also some lessening of the ability to distinguish yellow from blue, which may progress until total color-blindness results $[59$, p. $141 ; 68$, p. 45$]$. The luminosity function is essentially the same as for normal vision [57], and white, gray, and black are perceived in a relatively normal way. Köllner [59, p. 148] says, "In the dichromatic state the patient sees in the spectrum only yellow and blue, and the yellow corresponds to the hue which is elicited in the normal eye by light of wavelength $575 \mathrm{~m} \mu$, the blue to the hue corresponding to that of wavelength $471 \mathrm{~m} \mu$." These facts, like those of peripheral vision by the normal eye, indicate that the deuteranopic observer sees a yellow like that corresponding to about $575 \mathrm{~m} \mu$ for the normal observer, and a blue corresponding to about $470 \mathrm{~m} \mu$.

\section{Unilateral Red-Green Blindness}

Unilateral inherited defects of vision are probably much more common than would be supposed from accounts of such cases appearing in the literature. An Army physician observed in 1920 [5], "It has been recently discovered that because a man has excellent color vision in one eye he does not necessarily have it in the other." And a recent estimate [66] places the incidence of unilateral defects at about 4 percent of the total color defectives. Since, however, color vision of both eyes is most frequently tested at the same time, it follows that most unilateral defects go unnoticed [58, 66, 88]. Table 2 lists chronologically all cases of unilateral defects in color vision that could be uncovered by a reasonable search of the literature. There are 40 original articles listed involving 37 cases, a case of unilateral protanopia being dealt with twice by Hippel [36, 37] and once by Holmgren [42], a case of unilateral tritanopia being dealt with both by Kirschmann [54] and by Dieter [13], a case of unilateral deuteranomaly both by v. Kries [62] and by Trendelenburg [94] and one article by Bonner [101] mentioning two cases previously unreported.

It should be noted that the skill and resources of the several investigators varied over a wide range, so that both the type of defect for the
TABLE 2. Unilateral defects of color vision

\begin{tabular}{|c|c|c|c|}
\hline Author & Date & Probable cause of defect & Type of defect \\
\hline & & 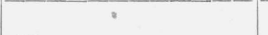 & \\
\hline $\begin{array}{l}\text { v. B a umgart- } \\
\text { ner [6]. }\end{array}$ & 1858 & Blow rn eye..... & $\begin{array}{l}\text { Temporary achroma- } \\
\text { topsia. }\end{array}$ \\
\hline Brische [11] ....... & 1862 & Attack of vertigo ...... & Temporary cyanopsia. \\
\hline Niemetscheck [77] & 1868 & $\begin{array}{l}\text { Skull-bone degenera- } \\
\text { tion. }\end{array}$ & $\begin{array}{l}\text { Tritanopia and chlo- } \\
\text { ropsia. }\end{array}$ \\
\hline Woinow [98] & 1871 & $\begin{array}{l}\text { Noticed after head- } \\
\text { wound, but perhaps } \\
\text { inherited. }\end{array}$ & Atypical, red phobia. \\
\hline Becker $[7] \ldots$ & 1879 & Inherited & Acromatopsia. \\
\hline Hippel [36] ..... & 1880 & $\ldots$..... do _.... & Protanopia. \\
\hline Holmgren [42] . . . & 1881 & _.... do ..... & Do. \\
\hline Hippel [37] ..... & 1881 & $\ldots$.......... & Do. \\
\hline Holmgren [44] .... & 1881 & _.... do & Tritanopia. \\
\hline Steffan $[90] \ldots . . .$. & 1881 & A poplexy ... & $\begin{array}{l}\text { Approach to achroma- } \\
\text { topsia. }\end{array}$ \\
\hline Snell $[88]_{-}$ & 1881 & Skull fracture.. & $\begin{array}{l}\text { Protanopia, deutera- } \\
\text { nomaly. }\end{array}$ \\
\hline Hermann [32] ..... & 1882 & Unknown .... & Tritanopia. \\
\hline Kolbe $[56] \ldots \ldots$ & 1882 & Inherited ..... & Red-green weakness. \\
\hline Shufeldt $[86] \ldots$ & 1883 & _._. do _._. & Red-brown confusion. \\
\hline Donders [16] ...... & 1884 & ..... do ........ & $\begin{array}{l}\text { Abnormal Rayleigh } \\
\text { equation. }\end{array}$ \\
\hline $\begin{array}{l}\text { E d r i d g e - } \\
\text { Green [18]. }\end{array}$ & 1889 & Probably retinitis..... & $\begin{array}{l}\text { Tritanopia, yellow- } \\
\text { blue weakness. }\end{array}$ \\
\hline Hering [31] ...... & 1890 & Optic-nerve atrophy - - & $\begin{array}{l}\text { Approach to deuter- } \\
\text { anopia. }\end{array}$ \\
\hline Hess [34] ...... & 1890 & Nerve injury & Do. \\
\hline Snell [89] ..... & 1892 & $\begin{array}{l}\text { Noticed after blow on } \\
\text { head, but perhaps } \\
\text { inherited. }\end{array}$ & Deuteranopia. \\
\hline Kirschmann [54] & 1893 & Inherited & Tritanopia. \\
\hline Hilbert [35] ...... & 1894 & do do & Slight abnormalities. \\
\hline Beevor $[8] \ldots$ & 1894 & Nerve injury & Achromatopsia. \\
\hline Piper [80] ........ & 1905 & ... do & $\begin{array}{l}\text { Achromatopsia, tritan- } \\
\text { opia. }\end{array}$ \\
\hline Samojloff [85] .. & 1906 & Inherited ....... & $\begin{array}{l}\text { Abnormal Rayleigh } \\
\text { equation. }\end{array}$ \\
\hline Köllner [57]_...... & 1909 & Optic-nerve atrophy -- & $\begin{array}{l}\text { Achromatopsia, tritan- } \\
\text { opia. }\end{array}$ \\
\hline Hayes $[28] \ldots$ & 1911 & Inherited & Protanomaly. \\
\hline Hegner [29] ....... & 1915 & _.... do & Do. \\
\hline Lohmann [65] ..... & 1917 & $\ldots$ do & $\begin{array}{l}\text { Abnormal contrast ef- } \\
\text { fects. }\end{array}$ \\
\hline v. Kries [62] ...... & 1919 & _. do & Deuteranomaly. \\
\hline Goldschmidt [26] & 1919 & Gunshot headwound.. & Protanomaly. \\
\hline Bonner $[10] \ldots . . .$. & 1923 & $\begin{array}{l}\text { Optic atrophy (tuber- } \\
\text { culous). }\end{array}$ & Green weakness. \\
\hline Jennings [48] & 1925 & $\begin{array}{l}\text { Noticed after blow on } \\
\text { head, but perhaps } \\
\text { inherited. }\end{array}$ & Red-green blindness. \\
\hline Bonner [11] - & $\begin{array}{l}1926 \\
1926\end{array}$ & Unknown ............. & Achromatopsia. \\
\hline Dieter [13] ....... & 1927 & Inherited & Tritanopia. \\
\hline $\begin{array}{l}\text { Miles, B e a u - } \\
\text { mont [66]. }\end{array}$ & 1931 & Unknown.... & Red-green blindness. \\
\hline Miles, Craig [67] & 1931 & & Do. \\
\hline Neipperg [75] .... & 1932 & Inherited & Do. \\
\hline $\begin{array}{l}\text { T r e n d e l e n - } \\
\text { berg [94]. }\end{array}$ & 1941 & ..... do ......... & Deuteranomaly. \\
\hline Sloan $[87] \ldots . . . . .$. & 1947 & ...... do & Deuteranopia. \\
\hline
\end{tabular}

defective eye and the degree of approach to the norm in cases having one supposedly normal eye are subject to various degrees of doubt. Similarly, in many cases the report of the probable cause of the defect is based on very incomplete data. If there were no evidence of disease or 
injury, the defect is listed as probably inherited. In one case, however, it is known definitely that the defect was inherited; the subject found by $\mathrm{v}$. Kries to have unilateral deuteranomaly was found later [94] to have transmitted a bilateral deuteroform defect to his daughter. Table 2 is intended to give brief summaries of all cases of unilateral defects of color vision, and the information is as precise as can be given in a few words; but these summaries are, of course, often unsatisfactory substitutes for the original articles that should be consulted if more detailed information is desired.

Since many of these cases are to be neglected as having no bearing on the color perceptions of protanopic and deuteranopic observers, it is convenient to introduce here the definitions of the terms by which the type of defect has been indicated in table 2 .

Trichromatism-a.type of vision in which the colors seen require in general three independently adjustable primaries (such as red, green, and blue) for their duplication by mixture. Normal vision is one form of trichromatism.

Dichromatism - a type of vision in which the colors seen require in general two independently adjustable primaries (such as red and green, or purple and yellow) for their duplication by mixture.

Monochromatism - a type of vision in which the colors seen require only a single adjustable primary to match them. Any light may serve as the primary.

Protanomaly - a type of trichromatism in which the relative luminosity function is too low at the longwave end to fall within normal limits, and in which an abnormally large proportion of red in a red-green mixture is required to match a given yellow.

Deuteranomaly - a type of trichromatism in which the relative luminosity function falls within "normal limits and in which an abnormally large proportion of green is required in a redgreen mixture to match a given yellow.

Protanopia - a type of dichromatism in which red and blue-green are confused, but no abnormal proportion of red plus green is required to match a given yellow, and the relative luminosity function is too low at the longwave end to fall within normal limits.

Deuteranopia - a type of dichromatism in which purplish red and green are confused, but no abnormal proportion of red and green is required to match a given yellow, and the relative luminosity function falls within normal limits.

Tritanopia-a type of dichromatism in which reddish blue and greenish yellow are confused.

Achromatopsia - a type of monochromatism in which all colors are perceived as neutral (such as black, gray, and white).

Chloropsia-green vision.

Cyanopsia-blue vision.

Rayleigh [83] equation - the proportions of red and green required in a mixture to match a given yellow. Usually spectrum red $(670 \mathrm{~m} \mu)$ is mixed with spectrum green $(535 \mathrm{~m} \mu)$ in such proportions as to match spectrum yellow (589 $\mathrm{m} \mu)$.

It would seem that only cases of protanopia and deuteranopia listed in table 2 need be considered, but it has often been noted [28, 62 (p. 148), 74] that under unfavorable conditions (such as small angular extent of observing field, reduced observing time [29], low field luminance, or dark surrounding field) protanomalous and deuteranomalous observers make the same mistakes as protanopes and deuteranopes, and respond as if they had dichromatic rather than trichromatic visual systems (red-green confusion, presence of a neutral point in the spectrum near $495 \mathrm{~m} \mu$, and so on). On this account, many color-perception tests fail to differentiate red-green confusers into groups of dichromats and trichromats. It is possible therefore to obtain information concerning the color perceptions characteristic of dichromatic visual systems from unilateral protanomaly and unilateral deuteranomaly, provided attention is paid to observations obtained by such observers under conditions that reduce these systems to, or nearly to, dichromatism. Table 3 lists all unilateral cases from which information regarding protanopic and deuteranopic color perceptions can be gleaned. It also includes a bilateral case reported by Nagel [72], which has a bearing on deuteranopic color perception. In this case the fovea was dichromatic, the periphery trichromatic, quite beyond the vestigial trace often reported by dichromats. Cases of unilateral achromatopsia and tritanopia listed in table 2 have been omitted, together with those of red-green confusion not giving information regarding color perceptions (Snell, 
Kolbe, Shufeldt, Hegner, Lohmann, Jennings, Neippert, Bonner, and Miles and Beaumont). Cases of slight abnormality not approaching dichromatism are also omitted (Donders, Hilbert, Samojloff).

The case of atypical unilateral sense disturbance reported by Woinow [98] has been omitted, because the report is contradictory. The subject was reported to be deuteranopic (green blind) in one eye, and measurements by rotating sector disks indicated confusion of both red and green with gray. There was, however, hysterical fear of red particularly from the eye which, as we have seen, under some conditions could not distinguish red from gray Furthermore, all objects seen by this eye were tinged with red, but spectrum yellow appeared blue. There is no evidence, except this latter rather confusing report, that the color system of the defective eye was dichromatic; it might have been monochromatic. Reports of the subject in other sense fields (taste, temperature, smell, hearing) indicated a profound psychic disturbance.

The case of temporary cyanopsia reported by Brische has also been omitted; the visual system seems to have been monochromatism, black, gray, and white objects, and all parts of the spectrum, appearing blue.

In table 3 the indicated perceptions are specified in many cases simply by color name, and we see, as before, that unilateral defects of vision indicate that the protanopic and deuteranopic perception of white is normal, and those of hue are some kind of a yellow and some kind of a blue. In other cases these indications have been made more exact by direct comparison of the spectrum colors viewed by the defective eye with those of the same spectrum viewed by the normal eye. Orange, for example, is seen as yellow by the dichromatic eye, as is also yellow green; but the part of the spectrum whose hue is the same for both the normal and the defective eye serves as a specification for the particular kind of yellow sensed by the dichromatic eye; that is, we can say from such evidence that the dichromatic yellow is like that perceived at some certain portion of the spectrum by the normal eye. Five of the entries in table 3 are of this nature. Four out of five of these entries refer to subjects who had in the defective eye slight, rather than vanishing, ability to distinguish red from green, but reports of hue difference between the two eyes could still be made and the spectral region yielding the same hue for both eyes determined. In general, it will be noted that for both the deuteranopic and protanopic forms the yellow is closely like that of the spectrum at $575 \mathrm{~m} \mu$ to the normal eye, and the blue like that of $470 \mathrm{~m} \mu$. This agrees with indications for the deuteranopic form obtained from the periphery of the normal retina and from acquired bilateral color-blindness.

The three exceptions to this generalization are, first, the wavelength $589 \mathrm{~m} \mu$ found by Hippel for his unilaterally color-blind observer; second, the designation, orange-yellow, shown for Goldschmidt's case of unilateral protanomaly; and third, the contradictory report by Sloan's unilateral deuteranope. The first two exceptions suggest that some protanopes, at least, see orangeyellow instead of the slightly greenish yellow of $575 \mathrm{~m} \mu$. Hippel, however, depended on the observation of flame spectra under the unfavorable condition of a dark background and did not report tests of any yellowish spectrum color other than $589 \mathrm{~m} \mu$ (that is, none nearer than this to $575 \mathrm{~m} \mu$ ), so his finding may merely be an imprecise check of $575 \mathrm{~m} \mu$. Furthermore, the report of orangeyellow is rendered doubtful by the report by Holmgren of greenish yellow ("greenish yellow, or citron yellow, not golden yellow") for the same case [42] examined by means of dyed wools. In the second exception, it was stated [26] rather inexplicitly that for the defective eye, "the green appeared in the spectrum stretched out into the yellow, and the blue stretched out into the violet, ..." Neither of these reports can be taken as established exceptions to the otherwise consistent indication that the yellow and blue of red-green confusers are, respectively, closely what the normal eye sees in the spectral regions near 575 and $470 \mathrm{~m} \mu .^{2}$

Some writers [28] have classified the Hering and Hess cases as probably protanomaly because of

\footnotetext{
2 Since the indicated perceptions for both protanopic and deuteranopic vision are not significantly different, no extended discussion of discrepancies in classification of the unilateral defects shown in table 3 is required. For the same reason, it is unimportant for the present purpose to determine whether the defect is inherited or acquired. However, it may be remarked that the classification by Hippel of his patient as what we now call deuteranopic rests on his failure to find the spectrum shortened on the long-wave end relative to that for the normal eye, all of the various spectral emission lines used being either visible to both eyes or invisible to both. The classification of protanopia, however, is firmly grounded not only on Holmgren's finding based upon the Holmgren wool test, which includes a chromaticity method of diagnosing protanopia, but also on Hippel's own report that the brightest part of the spectrum for the defective eye was shifted considerably toward the short-wave end relative to that for the normal eye.
} 
the statement that the long-wave end of the spectrum was shortened. This minor shortening is to be expected because of the partial impairment of the yellow-blue and light-dark senses, as well as the almost complete disappearance of the red-green sense. It is not to be confused [68, (p. 45)] with the much larger departure from normal characteristic of protanopia, designated by Hering as "blue-sighted red-green blindness."

The reclassification of Hayes' subject from protanopia to protanomaly is based upon the fact that the normal setting of a red plus a green by disk mixture to match a yellow plus black plus white was found by the subject's defective eye to be much too green. To satisfy the defective eye, she had to put by far more red in the first mixture. Hayes' conclusions are sound if translated into accepted terminology.

A further word is required regarding the apparently contradictory report by Sloan's unilateral deuteranope [87] that, on the one hand, the hues seen are like Munsell 5Y 5/5 (dominant wavelength $575 \mathrm{~m} \mu$ ) and $3 \mathrm{~PB} 5 / 5$ (dominant wavelength $478 \mathrm{~m} \mu$ ), and that, on the other they are like the spectrum at 584 and $452 \mathrm{~m} \mu$. There are several possibilities to be explored, but it will be sufficient for the present to point out that if both eyes of this subject had an abnormally large amount of ocular pigmentation and so were provided with a permanent adaptation to reddish yellow, the discrepancy would be explained. The same explanation would also account for the discrepancy between the wavelength of neutral point estimated from the Munsell 5/5 locus (498 $\mathrm{m} \mu$, which is typical of deuteranopia $[50,51])$ and that estimated directly from the spectrum (503 $\mathrm{m} \mu$, corresponding to reddish yellow adaptation). A more complete study of this observer might be worth while.

\section{Agreement with Theories of Color Vision}

The evidence just reviewed that red-green confusers see yellow and blue does not, of course, prove that all red-green confusers have those color perceptions, though the absence of reliable conflicting evidence renders such a conclusion highly probable. Thus, Parsons [79, p. 191] remarked in 1924, "Most observers think that the two sensations experienced (by protanopes and deuteranopes) correspond most closely to normal yellow and blue. Uncomplicated cases of uni- lateral congenital colour blindness would afford valuable evidence ...". Similarly, in 1927, Dieter [13, p. 79] said, "Only if still more cases of the same kind are completely analysed will there be sufficient ground for the alluring possibility of theoretical appraisal." Unilateral color-blindness, however, has already had an important influence on color theory.

During the period (1880-1920) when the experimental facts of protanopic and deuteranopic color perceptions were being established, the controversy between the three-components (YoungHelmholtz) theory of color vision and opponentcolors (Hering) theory reached its height [64].

According to the three-components theory, there are in the normal retina three independent photosensitive mechanisms, one sensitive preponderantly to the long-wave portion of the spectrum and yielding a red response, another sensitive preponderantly to the middle portion of the spectrum and yielding a green response, and the third sensitive preponderantly to the shortwave portion of the spectrum and yielding a blue or violet response.

According to the opponent-colors theory, there are in the normal visual mechanism three pairs of opposing processes, a black-white pair, which cancels to leave gray, and two chromatic pairs, blue-yellow, and red-green, which cancel to gray when both members of the pair are excited equally.

Red-green blindness according to the threecomponents theory comes from failure of either the red-component (protanopia) or the greencomponent (deuteranopia), leading to the indication that protanopes must see only mixtures of green and violet, and deuteranopes, only mixtures of red and violet. But, by the opponent-colors theory, only one type of red-green blindness is to be expected, that arising from elimination of the red-green process, leaving the perceptions white and black, blue and yellow.

The case of unilateral protanopia (Hippel, Holmgren, table 3 ) was a blow to the original form of three-components theory. It showed that one red-green blind eye, at least, saw yellow and blue, not green and violet. The three-components theory can be thrown into a form accounting both conveniently and precisely for the red-green confusions of protanopes and deuteranopes [51], but this single case of unilateral color-blindness showed that it is not a reliable guide to what they 
TABLE 3. Cases of unilateral defect of vision giving information regarding protanopic and deuteranopic color perceptions

\begin{tabular}{|c|c|c|c|c|c|}
\hline \multirow{2}{*}{ Author } & \multirow{2}{*}{ Date } & \multicolumn{2}{|c|}{ Classification of the defect } & \multirow{2}{*}{\multicolumn{2}{|c|}{ Indicated perceptions }} \\
\hline & & Author's own & Present & & \\
\hline Hippel [36] .... & $1880 \ldots$ & Deuteranopia. & Protanopia & Yellow .... & Blue. \\
\hline Holmgren [42] & $1881 \ldots$ & Protanopia & $\ldots \mathrm{do} \ldots \ldots . . . . .$. & Greenish yellow.... & Violet blue. \\
\hline Hippel [37[ . . . & $1881 \ldots$ & Deuteranopia................... & do do & Like $589 \mu$ & Blue. \\
\hline Hering $[31] \ldots$ & $1890 \ldots$ & Approach to deuteranopia & Approach to deuteranopia & Yellow & Do. \\
\hline Hess [34] .... & $1890 \ldots$ & do & do do & Like $575 \mu \ldots \ldots$ & Like $471 \mathrm{~m}_{\mu}$. \\
\hline Nagel [72] ... & $1905 \ldots$ & Deuteranopia..... & Deuteranopia & Yellow _........ & Blue. \\
\hline Hayes [28] ... & $1911 \ldots$ & Protanopia & Protanomaly & . do & Do. \\
\hline จ. Kries [62] ...... & $1919 \ldots$ & Deuteranomaly ... & Deuteranomaly _... & Like $573 \mu \ldots \ldots$ & Like 464 to $480 \mathrm{~m}_{\mu}$. \\
\hline Goldschmidt [26]. & $1919 \ldots$ & Protanomaly & Protanomaly ...... & Orange, yellow & Blue. \\
\hline Sloan [87] $\ldots . .$. & $1947 \ldots$ & Deuteranopia..... & Deuteranopia $\ldots \ldots$ & Like $5 \mathrm{Y} 5 / 5$, also $584 \mathrm{~m} \mu \ldots$ & Like $3 \mathrm{~PB}, 5 / 5$, also $452 \mathrm{~m} \mu$. \\
\hline
\end{tabular}

see. The opponent-colors theory, on the other hand, received dramatic support from the color perceptions reported for this case. This latter theory, however, does not account for two forms of red-green blindness, but only one, deuteranopia.

It may be said, therefore, that both of these simple visual theories received fatal blows from discovery of the facts of protanopia and deuteranopia. Some advocates of the three-components theory refused to be impressed by the HippelHolmgren case of unilateral protanopia. Holmgren, himself, continued to use the terms, "red blind", "green blind" and "violet blind", and offered a prize of 400 crowns to anyone who would bring to his attention a case of unilateral "green blindness" [45]. Some three-component advocates took refuge in Fick's [21, 22] suggestion that redgreen blindness results, not from the complete failure of one of the three components, the red or the green, but from the receptors for red and green having identical photo-sensitive substances, either that normally used for red or that normally used for green. Other advocates of the three-components theory (König, v. Kries) eventually took up the very similar theoretical position [54a, 60 (p. 169)] originally proposed by Donders [15] that the three-components theory holds for processes in one stage or zone of the visual mechanism (perhaps the photosensitive substances), whereas the opponent-colors theory holds for processes in a later stage or zone (perhaps the optic nerve). This view is known as the zone or stage theory of vision. Furthermore, a very able advocate of the opponent-colors theory, G. E. Müller, adopted a theoretical view which, although divergent in detail and more elaborated, was essentially in agreement with the zone theories favored by Donders, König, and v. Kries. All of these theories allow for protanopic and deuteranopic perception of yellow and blue, though that of Müller does not require it for protanopes. Müller remarks (p. 49), "If the color-blind is a deuteranope, his chromatic color sensations must therefore (by this theory) be pure yellow and pure blue . . . . It is otherwise with protanopes. If in an individual outer red-green sensitivity is absent, it makes no difference in his behavior, either in practical living or in any investigation, whether the remaining greenish-yellow and reddish-blue processes excite only yellow and blue in the optic nerve, or whether, . . ., they excite greenishyellow or reddish-blue sensations, or whether, . . . they excite only green and red, or . . . even only green and blue, or . . . yellow and red. An individual lacking outer red-green sensitivity will give exactly the same color equations, exactly the same spectral positions of brightness maximum and neutral point regardless of whether his greenish yellow and reddish blue processes evoke nerve excitations corresponding to the like-named excitations (yellow and blue) or unlike-named excitations (green and red), or by simultaneous action of both sorts, excitations of exactly the same kind as the processes themselves. We would therefore not be particularly astonished if it were established definitely in a unilateral case of protanopia that the yellow sensation experienced actually was somewhat greenish and that the blue sensation experienced actually was somewhat reddish, and we would not believe that the ground was disappearing under our feet if sometime there should occur a (quite improbable) case establishing 
the two chromatic color sensations of a protanopic eye as the pure-green sensation and the pure-red sensation."

Since all theories of vision with any pretense of being complete provide for the perception of yellow and blue by red-green confusers, and since a reasonable search of the literature has failed to uncover any reliable evidence against this indication, it is proposed to build a method of designating protanopic and deuteranopic perceptions of surface colors on the finding that such observers see a yellow and a blue like those seen by the normal observer in the spectrum near 575 and $470 \mathrm{~m} \mu$, respectively. The consequences of this choice of hues are bound to appear correct to binocular red-green confusers. These consequences can be contradicted only by observers having unilateral defects; and the probability of such contradiction arising may be estimated from the fact that the eight cases recorded so far (see table 3 ) fail to contradict it.

\section{Derivation of Deuteranopic and Protanopic Munsell Notations}

Object-color perceptions by normal observers are most commonly described in terms of the attributes, hue, lightness, and saturation [78] defined [2] as follows:

"Hue is the attribute which determines whether the color perception is red, yellow, green, blue, purple, or the like.

"Lightness is the attribute which permits an object-color perception to be classified as equivalent to some member of the series of grays ranging between black and white.

"Saturation is the attribute of an object-color perception which determines the degree of its difference from the gray of the same lightness."

The Munsell color system is based upon this method of description. Munsell hue, value, and chroma are intended to correspond to hue, lightness, and saturation, respectively, and the samples in the Munsell Book of Color [70] when viewed in daylight by a normal observer under usual viewing conditions (daylight adapted eye, light to mediumgray surrounding field) yield color perceptions such that Munsell hue correlates closely with the perceived hue, Munsell value with the perceived lightness, and Munsell chroma with the perceived saturation. A method has been developed [49] by means of which readily understood color names may be found for any color from its Munsell notation.

When the samples of the Munsell Book of Color are viewed by a protanopic or a deuteranopic observer, however, the color perceptions obtained are not even approximately indicated by the Munsell notation. It is proposed to develop a method of deriving protanopic and deuteranopic Munsell notations, analogous to the present normal Munsell notations, that will correlate with the color-perceptions that red-green-blind observers obtain from objects viewed in daylight uuder usual conditions. It has been shown [51] that the color confusions of red-green-blind observers can be found from three numbers $\left(K, W_{p}, W_{d}\right)$ related to the tristimulus values $(X, Y, Z)$ of the ICI standard observer by the transformation equations :

$$
\left.\begin{array}{l}
K=Z, \\
W_{p}=-0.460 X+1.359 Y+0.101 Z, \\
W_{d}=Y .
\end{array}\right\}
$$

Any two colors having identical values of $K$ and $\mathrm{W}_{p}$ are identical to the average protanope; those having identical values of $K$ and $\mathrm{W}_{d}$ are identical to the average deuteranope. Figure 1 shows in arbitrary units the values of $K, \mathrm{~W}_{p}$, and $\mathrm{W}_{d}$ for all parts of a spectrum of unit irradiance per unit wavelength.

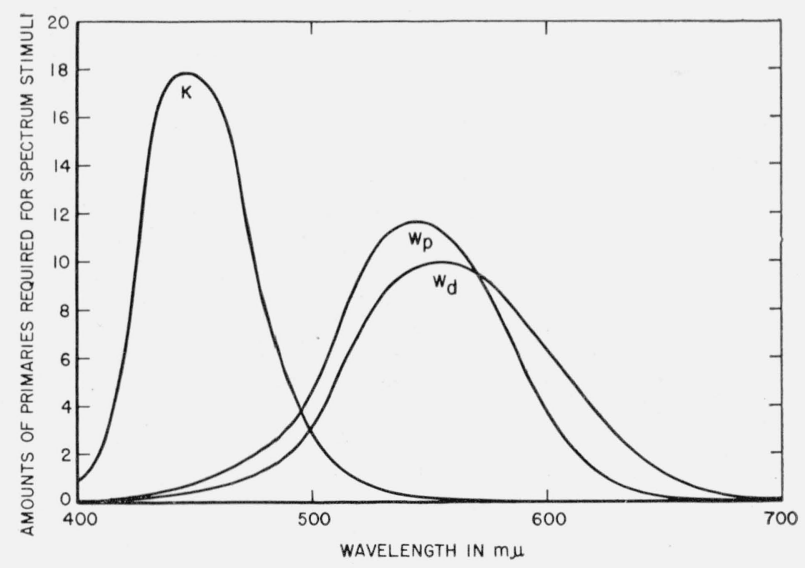

Figure 1

One of each of the two pairs of numbers $\left(W_{d}\right.$, $\mathrm{W}_{p}$ ), used in judging equivalence of colors for deuteranopic and protanopic observers, refers to the luminous aspect of the color (luminance for a self-luminous area, luminous directional reflect- 
ance for a surface), the other number, $K$, of each pair taken relative to the first refers to the chromatic aspect. For the ICI observer, luminous directional reflectance is customarily expressed relative to that $\left(Y_{0}\right)$ of magnesium oxide. By analogy we take $W_{d} /\left(W_{d}\right)_{0}=Y / Y_{0}$ for deuteranopic luminous directional reflectance, and

$$
\frac{W_{p}}{\left(W_{p}\right)_{0}}=\frac{-0.460 X+1.359 Y+0.101 Z}{-0.460 X_{0}+1.359 Y_{0}+0.101 Z_{0}},
$$

for protanopic luminous directional reflectance. But since for magnesium oxide it is customary to take $X_{0}=0.9804, Y_{0}=1.0000$, and $Z_{0}=1.1512$, protanopic luminous directional reflectance may be written simply as $0.9733 W_{p}$.

It is customary to specify the chromatic aspect of a color for the ICI standard observer by means of trichromatic coordinates, or chromaticity coordinates $x, y$, and $z$. By analogy with this practice, the chromaticity coordinates for deuteranopic and protanopic observers are the dichromatic coordinates $\left(w_{d}, k_{d} ; w_{p}, k_{p}\right)$ defined as follows:

$$
\left.\begin{array}{r}
w_{d} \equiv W_{d} /\left(W_{d}+K\right)= \\
Y /(Y+Z)=y /(y+z)=y /(1-x), \\
k_{d} \equiv K /\left(W_{d}+K\right)=Z /(Y+Z)= \\
z /(y+z)=(1-x-y) /(1-x) .
\end{array}\right\}
$$

From eq $2 \mathrm{~d}$ and $2 \mathrm{p}$ it will be noted that $w_{p}$ and $k_{p}$ sum to unity; likewise, $w_{d}$ and $k_{d}$. Hence only one of the dichromatic chromaticity coordinates need be used. We will use $w_{d}$ and $w_{p}$, which may be taken as measures of the deuteranopic and protanopic "warm" quality, respectively. According to the data and views already summarized in sections II and III, it is legitimate to take them more precisely as indications of "yellowness."

Since for the approximately 400 samples of the Munsell Book of Color, the tristimulus values, $X, Y, Z$, have already been evaluated from spectrophotometric measurements $[23,53]$, it is a simple matter to compute $W_{d} /\left(W_{d}\right)_{0}$ and $w_{d}$ from eq 1 and $2 \mathrm{~d}$, and so arrive at a specification of deuteranopic luminous directional reflectance and chromaticity. The results are shown in table 4. Similarly there have been computed for these 400 samples values of $W_{p} /\left(W_{p}\right)_{0}$ and $w_{p}$, which serve as specifications of protanopic luminous directional reflectance and chromaticity, respectively. These results are shown in table 5 . In both tables 4 and 5 , the samples have been arranged in a sequence, which would appear orderly to the average deuteranope (table 4) or protanope (table 5). The samples were first arranged according to reflectance, and, as expected, for deuteranopic vision were found to form seven groups of nearly constant reflectance (one for each of the Munsell values 2 to 8) with definite reflectance gaps separating them. The samples of each group are arranged according to yellowness, approximately as indicated by the chromaticity coordinate, $w_{d}$. Also, as expected, no such grouping was found after arranging the samples according to protanopic reflectance. However, the samples were arranged arbitrarily into 14 groups (one for each of the Munsell values $2.0,2.5, \ldots 8.5)$ so that the protanopic reflectance of each sample in one group is both lower than all of those in the group above and higher than all of those in the group below. Within each of these 14 groups the samples were arranged according to yellowness, approximately as indicated by the chromaticity coordinate $w_{p}$.

From the first three columns of tables 4 and 5 , it may be seen that an arrangement that seems orderly to a deuteranope or protanope may contain outstanding irregularities to a normal observer. For example, the first four entries in table 4 are $\mathrm{Y} 8 / 12, \mathrm{Y} 8 / 10,10 \mathrm{Y} 8 / 8, \mathrm{Y} 8 / 8$. The second column indicates that these samples all have reflectances close to 0.58 ; that is, the colors of these surfaces appear to the average deuteranope as well as to the average normal observer to be about equally light. The third column indicates that the first sample is the yellowest and fourth the least yellow. With the exception of the third sample (10Y 8/8), this classification accords with that of the normal observer, which is indicated closely by the Munsell notation (Hue Value/ Chroma) Y 8/12, Y 8/10, Y 8/8. The third sample, however, is a more greenish yellow than the others and for the normal observer does not 
fit into the series. In this instance table 4 has served to indicate with some precision the kind of greenish yellow that the average deuteranope may be expected to confuse with a certain group of yellows. It indicates in general exactly the red-green confusions characteristic of deuteranopia. Table 5 indicates in a similar way the red-green confusions characteristic of protanopia.

TABLE 4. Deuteranopic arrangement

\begin{tabular}{|c|c|c|c|c|}
\hline \multirow{2}{*}{$\begin{array}{l}\text { Munsell } \\
\text { book nota- } \\
\text { tion }\end{array}$} & \multirow{2}{*}{$\begin{array}{l}\text { Deuteran- } \\
\text { opic re- } \\
\text { flectance, } \\
W_{d}=Y\end{array}$} & \multirow{2}{*}{$\begin{array}{c}\text { Deuteran- } \\
\text { opic chro- } \\
\text { maticity } \\
\text { coordinate, } \\
w_{d}\end{array}$} & \multicolumn{2}{|c|}{ Munsell renotations } \\
\hline & & & Deuteranopic & Normal \\
\hline $5 Y 8 / 12$ & 0.5706 & 0.8911 & $5 \mathrm{Y} 7.9 / 12.2$ & 5. $5 \mathrm{Y} \quad 7.9 / 12.3$ \\
\hline $5 \mathrm{Y} 8 / 10$ & .5784 & .8578 & $5 \mathrm{Y} 7.9 / 11.0$ & 5. $5 \mathrm{Y} \quad 7.9 / 11.1$ \\
\hline $10 \mathrm{Y} 8 / 8$ & .6035 & .7918 & $5 Y 8.1 / 9.2$ & $0.5 \mathrm{GY} 8.1 / 9.1$ \\
\hline $5 \mathrm{Y} 8 / 8$ & .5794 & .7660 & $5 \mathrm{Y} 7.9 / 8.2$ & $5.5 \mathrm{Y} \quad 7.9 / 8.2$ \\
\hline $5 \mathrm{Y} 8 / 8$ & .5732 & .7606 & $5 \mathrm{Y} 7.9 / 8.1$ & $5.5 \mathrm{Y} \quad 7.9 / 8.3$ \\
\hline $5 \mathrm{GY} 8 / 8$ & .6022 & .7464 & $5 Y 8.1 / 7.9$ & 4. $5 \mathrm{GY} 8.1 / 8.7$ \\
\hline 10 YR $8 / 8$ & .6049 & .7064 & $5 Y 8.1 / 6.9$ & $0.5 \mathrm{Y} 8.1 / 7.6$ \\
\hline $5 \mathrm{Y} 8 / 6$ & .5784 & .6681 & $5 Y \quad 7.9 / 5.7$ & $4.5 \mathrm{Y} \quad 7.9 / 5.8$ \\
\hline $10 \mathrm{Y} 8 / 6$ & .5996 & .6664 & $5 Y 8.0 / 5.7$ & $0.5 \mathrm{GY} 8.0 / 5.7$ \\
\hline $5 \mathrm{GY} 8 / 6$ & .5929 & .6605 & $5 Y 8.0 / 5.5$ & 5. $5 \mathrm{GY} 8.0 / 6.2$ \\
\hline $10 \mathrm{YR} 8 / 6$ & .6114 & .6263 & $5 \mathrm{Y} 8.1 / 4.7$ & 9.0 YR $8.1 / 5.5$ \\
\hline $5 \mathrm{Y} 8 / 4$ & .5651 & .5851 & $5 \mathrm{Y} 7.8 / 3.4$ & $4.0 \mathrm{Y} 7.8 / 3.5$ \\
\hline $10 \mathrm{Y} 8 / 4$ & .5997 & .5827 & $5 Y 8.0 / 3.4$ & 1.0GY $8.0 / 3.4$ \\
\hline $10 \mathrm{GY} 8 / 6$ & .5793 & .5720 & $5 Y \quad 7.9 / 3.1$ & 9.5GY $7.9 / 5.1$ \\
\hline $5 \mathrm{GY} 8 / 4$ & .6041 & .5719 & $5 \mathrm{Y} 8.1 / 3.2$ & 6. $0 \mathrm{GY} 8.1 / 3.6$ \\
\hline 10 Y R $8 / 4$ & .5972 & .5579 & $5 \mathrm{Y} 8.0 / 2.8$ & 9.0 YR $8.0 / 3.4$ \\
\hline $10 \mathrm{GY} 8 / 4$ & .6181 & .5406 & $5 \mathrm{Y} 8.2 / 2.3$ & $0.5 \mathrm{G} 8.2 / 4.0$ \\
\hline $5 \mathrm{YR} 8 / 4$ & .5970 & .5311 & $5 \mathrm{Y} 8.0 / 2.0$ & 4.0 YR $8.0 / 3.5$ \\
\hline $10 \mathrm{R} 8 / 4$ & .6115 & .5156 & $5 \mathrm{Y} 8.1 / 1.6$ & 10.0R 8.1/3.7 \\
\hline bY $8 / 2$ & .5570 & .5141 & $5 \mathrm{Y} 7.8 / 1.5$ & $3.5 \mathrm{Y} 7.8 / 1.6$ \\
\hline $5 \mathrm{GY} 8 / 2$ & .5851 & .5107 & $5 \mathrm{Y} 8.0 / 1.5$ & $6.0 \mathrm{GY} 8.0 / 1.6$ \\
\hline $5 \mathrm{G} 8 / 6$ & .5595 & .4964 & $b \mathrm{Y} 7.8 / 1.1$ & $6.5 \mathrm{G} 7.8 / 4.7$ \\
\hline $5 \mathrm{YR} 8 / 2$ & .5917 & .4949 & $5 Y 8.0 / 1.0$ & 4.0YR $8.0 / 2.0$ \\
\hline $5 \mathrm{G} 8 / 4$ & .5881 & .4899 & $5 Y \quad 8.0 / 0.9$ & $5.5 \mathrm{G} 8.0 / 3.3$ \\
\hline $5 \mathrm{G} 8 / 2$ & .5694 & .4847 & $5 Y \quad 7.9 / 0.8$ & 4. $5 \mathrm{G} \quad 7.9 / 2.1$ \\
\hline $5 \mathrm{R} 8 / 4$ & .5862 & .4834 & $5 \mathrm{Y} 8.0 / 0.7$ & $35 \mathrm{R} 8.0 / 3.6$ \\
\hline $10 \mathrm{G} 8 / 2$ & .5965 & .4806 & $5 \mathrm{Y} 8.0 / 0.6$ & $5.0 \mathrm{G} 8.0 / 2.0$ \\
\hline N 8/ & .5751 & .4700 & $5 Y \quad 7.9 / 0.4$ & 2.5GY $7.9 / 0.3$ \\
\hline $5 \mathrm{R} 8 / 2$ & .6021 & .4699 & $5 \mathrm{Y} \quad 8.1 / 0.4$ & 2. $0 \mathrm{R} 8.1 / 2.2$ \\
\hline 10RP 8/ 6 & .6251 & .4693 & $5 \mathrm{Y} \quad 8.2 / 0.4$ & 8. $5 \mathrm{RP} 8.2 / 3.6$ \\
\hline $10 \mathrm{RP} 8 / 4$ & .6107 & .4678 & $5 \mathrm{Y} \quad 8.1 / 0.3$ & 9.5RP $8.1 / 2.7$ \\
\hline $5 \mathrm{BG} 8 / 2$ & .6082 & .4656 & $5 \mathrm{Y} 8.1 / 0.3$ & 1. $0 \mathrm{BG} 8.1 / 2.0$ \\
\hline $5 \mathrm{RP} 8 / 2$ & .6435 & .4633 & $5 \mathrm{Y} 8.3 / 0.2$ & 8.0RP $8.3 / 1.9$ \\
\hline $5 \mathrm{RP} 8 / 6$ & .6058 & .4611 & $5 \mathrm{Y} 8.1 / 0.1$ & 6.5RP 8.1/ 3.4 \\
\hline $5 \mathrm{RP} 8 / 4$ & .6050 & .4588 & $5 Y \quad 8.1 / 0.1$ & 5. $5 \mathrm{RP} 8.1 / 2.8$ \\
\hline $10 \mathrm{BG} 8 / 2$ & .6258 & .4557 & $5 \mathrm{~PB} 8.2 / 0.4$ & $5.0 \mathrm{BG} 8.2 / 1.9$ \\
\hline $5 \mathrm{~B} 8 / 2$ & .6179 & .4361 & $5 \mathrm{~PB} 8.2 / 1.7$ & 4. $0 \mathrm{~B} \quad 8.2 / 2.2$ \\
\hline $5 \mathrm{P} 8 / 2$ &. .5897 & .4330 & $5 \mathrm{~PB} 8.0 / 1.8$ & $5.0 \mathrm{P} 8.0 / 2.5$ \\
\hline $10 \mathrm{P} 8 / 4$ & .6182 & .4289 & $5 \mathrm{~PB} 8.2 / 2.2$ & 7. $5 \mathrm{P} \quad 8.2 / 3.5$ \\
\hline $5 \mathrm{~B} 8 / 4$ & .6025 & .4272 & $5 \mathrm{~PB} 8.1 / 2.3$ & 4. $0 \mathrm{~B} \quad 8.1 / 2.9$ \\
\hline $10 \mathrm{~B} 8 / 2$ & .6514 & .4170 & $5 \mathrm{~PB} 8.3 / 3.1$ & 1. $0 \mathrm{~PB} 8.3 / 3.0$ \\
\hline $5 \mathrm{~PB} 8 / 2$ & .6380 & .4139 & 5 PB $8.3 / 3.2$ & $5.0 \mathrm{~PB} 8.3 / 3.2$ \\
\hline $10 \mathrm{~PB} 8 / 2$ & .6499 & .4099 & 5 PB $8.3 / 3.4$ & 7. $5 \mathrm{~PB} 8.3 / 3.4$ \\
\hline P $8 / 4$ & .5940 & .4004 & $5 \mathrm{~PB} 8.0 / 3.9$ & 4. $5 \mathrm{P} 8.0 / 4.8$ \\
\hline
\end{tabular}

TABLE 4. Deuteranopic arrangement-Continued

\begin{tabular}{|c|c|c|c|c|}
\hline \multirow{2}{*}{$\begin{array}{l}\text { Munsell } \\
\text { book nota- } \\
\text { tion }\end{array}$} & \multirow{2}{*}{$\begin{array}{c}\text { Deuteran- } \\
\text { opic re- } \\
\text { flectance, } \\
W_{d}=Y\end{array}$} & \multirow{2}{*}{$\begin{array}{c}\text { Deuteran- } \\
\text { opic chro- } \\
\text { maticity } \\
\text { coordinate, } \\
w_{d}\end{array}$} & \multicolumn{2}{|c|}{ Munsell renotations } \\
\hline & & & Deuteranopic & Normal \\
\hline Y $7 / 10$ & 0.4189 & 0.8502 & $5 \mathrm{Y} 6.9 / 9.7$ & $5.0 \mathrm{Y} 6.9 / 9.7$ \\
\hline Y $7 / 8$ & .4220 & .8430 & $5 Y \quad 6.9 / 9.6$ & $6.0 \mathrm{Y} 6.9 / 9.5$ \\
\hline $10 \mathrm{YR} 7 / 10$ & .4179 & .8405 & $5 Y 6.9 / 9.4$ & 1.0Y $6.9 / 10.5$ \\
\hline $10 \mathrm{Y} 7 / 8$ & .4440 & .8358 & $5 Y 7.1 / 9.5$ & $10.0 Y \quad 7.1 / 9.3$ \\
\hline GY $7 / 10$ & .4263 & .8160 & $5 Y 7.0 / 9.0$ & 4. 0 GY $7.0 / 9.5$ \\
\hline YR $7 / 10$ & .4178 & .7614 & $5 Y 6.9 / 7.4$ & 5. 5 YR $6.9 / 10.1$ \\
\hline $10 \mathrm{YR} 7 / 8$ & .4397 & .7571 & $5 Y 7.1 / 7.4$ & $0.5 \mathrm{Y} 7.1 / 8.3$ \\
\hline GY $7 / 8$ & .4512 & .7437 & $5 Y 7.1 / 7.1$ & 5. $0 \mathrm{GY} 7.1 / 80$ \\
\hline $10 \mathrm{Y} 7 / 6$ & .4334 & .7406 & $5 Y 7.0 / 7.0$ & $0.5 \mathrm{GY} \quad 7.0 / 6.9$ \\
\hline Y 7/ 6 & .4158 & .7286 & $5 \mathrm{Y} 6.9 / 6.4$ & 4. 5 Y $6.9 / 6.6$ \\
\hline GY 7/ 6 & 4509 & .6867 & $5 \mathrm{Y} 7.1 / 5.7$ & $5.0 \mathrm{GY} \quad 7.1 / 6.4$ \\
\hline YR $7 / 8$ & .4356 & .6851 & $5 Y 7.0 / 5.6$ & 4. 5 YR $7.0 / 8.2$ \\
\hline $10 \mathrm{YR} 7 / 6$ & .4347 & .6818 & $5 \mathrm{Y} 7.0 / 5.5$ & 10.0 Y R $7.0 / 6.3$ \\
\hline $10 \mathrm{GY} 7 / 8$ & .4399 & .6509 & $5 Y 7.1 / 4.8$ & $10.0 \mathrm{GY} \quad 7.1 / 8.3$ \\
\hline YR 7/ 6 & .4262 & .6143 & $5 Y 7.0 / 3.9$ & 3.5YR $7.0 / 6.0$ \\
\hline YR 7/ 6 & .4180 & .6134 & $5 \mathrm{Y} 6.9 / 3.8$ & 3. 5 YR $6.9 / 5.9$ \\
\hline Y $7 / 4$ & .4282 & .6084 & $5 Y 7.0 / 3.7$ & $4.0 \mathrm{Y} 7.0 / 3.8$ \\
\hline $10 \mathrm{R} 7 / 8$ & .4290 & .6071 & $5 Y 7.0 / 3.7$ & $10.0 \mathrm{R} \quad 7.0 / 7.5$ \\
\hline $10 \mathrm{Y} 7 / 4$ & .4439 & .6053 & $5 Y 7.1 / 3.6$ & 1. $0 \mathrm{GY} 7.1 / 3.6$ \\
\hline $10 \mathrm{GY} 7 / 6$ & .4507 & .6046 & $5 Y 7.1 / 3.6$ & 10.0GY $7.1 / 6.5$ \\
\hline GY 7/ 4 & .4369 & .5910 & $5 Y 7.0 / 3.3$ & 5.5GY $7.0 / 3.8$ \\
\hline $10 \mathrm{YR} 7 / 4$ & .4602 & .5848 & $5 Y 7.2 / 2.5$ & $9.0 \mathrm{YR} 7.2 / 3.8$ \\
\hline YR 7/ 4 & .4376 & .5567 & $5 Y 7.0 / 2.5$ & 3.0YR $7.0 / 4.1$ \\
\hline $10 \mathrm{R} 7 / 6$ & .4550 & .5559 & $5 Y 7.2 / 2.5$ & 9.0R $7.2 / 5.7$ \\
\hline $10 \mathrm{GY} 7 / 4$ & .4571 & .5486 & $5 Y 7.2 / 2.3$ & 10.0GY $7.2 / 4.1$ \\
\hline $10 \mathrm{R} 7 / 4$ & .4537 & .5316 & $5 Y 7.2 / 1.8$ & 8.5R $7.2 / 4.7$ \\
\hline Y $7 / 2$ & .4454 & .5199 & $5 \mathrm{Y} 7.1 / 1.6$ & 4. $0 \mathrm{Y} 7.1 / 1.6$ \\
\hline R 7/ 8 & .4244 & .5188 & $5 Y 7.0 / 1.7$ & 3. $5 \mathrm{R} 7.0 / 7.0$ \\
\hline GY 7/ 2 & .4261 & .5177 & $5 Y 7.0 / 1.7$ & 5. $5 \mathrm{GY} \quad 7.0 / 1.8$ \\
\hline R 7/ 6 & .4452 & .5099 & $5 Y \quad 7.1 / 1.3$ & 3. $5 \mathrm{R} \quad 7.1 / 5.7$ \\
\hline YR $7 / 2$ & .4375 & .5058 & $5 Y 7.0 / 1.2$ & 3.0YR $7.0 / 2.1$ \\
\hline G $7 / 6$ & .4381 & .5030 & $5 \mathrm{Y} 7.0 / 1.1$ & $7.0 \mathrm{G} 7.0 / 6.1$ \\
\hline R $7 / 4$ & .4402 & .4973 & $5 Y 7.1 / 1,0$ & 3.0R $7.1 / 5.2$ \\
\hline G $7 / 4$ & .4340 & .4948 & $5 Y 7.0 / 0.9$ & $6.5 \mathrm{G} 7.0 / 4.3$ \\
\hline 10RP 7/ 8 & .4412 & .4801 & $5 Y 7.1 / 0.6$ & 9.0RP $7.1 / 5.8$ \\
\hline G $7 / 2$ & .4231 & .4777 & $5 \mathrm{Y} 7.0 / 0.5$ & $6.5 \mathrm{G} 7.0 / 2.1$ \\
\hline 10RP 7/ 6 & .4551 & .4773 & $5 Y 7.2 / 0.5$ & 9.5RP $7.2 / 4.7$ \\
\hline $5 \mathrm{R} 7 / 2$ & .4521 & .4766 & $5 Y 7.2 / 0.5$ & 2. $5 \mathrm{R} \quad 7.2 / 2.6$ \\
\hline $10 \mathrm{G} 7 / 4$ & .4672 & .4734 & $5 Y \quad 7.2 / 0.4$ & $0.5 \mathrm{BG} \quad 7.2 / 3.9$ \\
\hline 10RP $7 / 4$ & .4397 & .4732 & $5 Y \quad 7.1 / 0.4$ & 9.5RP $7.1 / 3.5$ \\
\hline RP 7/ 2 & .4727 & .4601 & $5 Y \quad 7.3 / 0.1$ & 6. $5 \mathrm{RP} \quad 7.3 / 2.3$ \\
\hline RP 7/ 4 & .4885 & .4590 & N 7.4/ & 6.0RP 7.4/ 3.3 \\
\hline BG $7 / 2$ & .4729 & .4589 & N 7.3/ & 3. $5 \mathrm{BG} 7.3 / 2.1$ \\
\hline N 7/ & .4433 & .4562 & 5 PB $7.1 / 0.2$ & 7. $5 \mathrm{~PB} 7.1 / 0.1$ \\
\hline RP 7/ 6 & .4955 & .4533 & $5 \mathrm{~PB} 7.4 / 0.3$ & 5.0RP $7.4 / 4.4$ \\
\hline BG 7/ 4 & .4634 & .4522 & $5 \mathrm{~PB} \quad 7.2 / 0.3$ & 4. $5 \mathrm{BG} 7.2 / 3.4$ \\
\hline $\mathrm{RP} 7 / 8$ & .4845 & .4494 & $5 \mathrm{~PB} \quad 7.4 / 0.4$ & 4.5RP $7.4 / 4.9$ \\
\hline B $7 / 2$ & .4718 & .4348 & $5 \mathrm{~PB} 7.3 / 1.5$ & 3.5B $7.3 / 2.2$ \\
\hline $10 \mathrm{P} 7 / 4$ & .4657 & .4285 & 5PB $7.2 / 1.9$ & 10.0P $7.2 / 4.0$ \\
\hline $10 \mathrm{BG} 7 / 4$ & .4555 & .4244 & $5 \mathrm{~PB} 7.2 / 2.2$ & $\begin{array}{lll}0.5 \mathrm{~B} & 7.2 / 3.6\end{array}$ \\
\hline $10 \mathrm{P} 7 / 6$ & .4675 & .4228 & $5 \mathrm{~PB} \quad 7.2 / 2.3$ & 9.5P $7.2 / 4.6$ \\
\hline P 7/ 2 & .4108 & .4206 & $5 \mathrm{~PB} 6.9 / 2.6$ & 4. $5 \mathrm{P} 6.9 / 2.7$ \\
\hline $10 \mathrm{P} 7 / 8$ & .4735 & .4125 & $5 \mathrm{~PB} \quad 7: 3 / 2.9$ & 9.5P $7.3 / 6.0$ \\
\hline PB 7/ 2 & .4907 & .4125 & $5 \mathrm{~PB} 7.4 / 3.0$ & $3.5 \mathrm{~PB} 7.4 / 3.0$ \\
\hline B 7/ 4 & .4575 & .4092 & $5 \mathrm{~PB} 7: 2 / 3.0$ & $5.0 \mathrm{~B} \quad 7.2 / 3.7$ \\
\hline $10 \mathrm{~PB} 7 / 4$ & .4498 & .3982 & 5PB $7.1 / 3.7$ & $10.0 \mathrm{~PB} 7.1 / 4.0$ \\
\hline
\end{tabular}


TABle 4. Deuteranopic arrangement-Continued

\begin{tabular}{|c|c|c|c|c|}
\hline \multirow{2}{*}{$\begin{array}{l}\text { Munsell } \\
\text { book nota- } \\
\text { tion }\end{array}$} & \multirow{2}{*}{$\begin{array}{c}\text { Deuteran- } \\
\text { opic re- } \\
\text { flectance, } \\
W_{d}=Y\end{array}$} & \multirow{2}{*}{$\begin{array}{c}\text { Deuteran- } \\
\text { opic chro- } \\
\text { maticity } \\
\text { coordinate, } \\
w_{d}\end{array}$} & \multicolumn{2}{|c|}{ Munsell renotations } \\
\hline & & & Deuteranopic & Normal \\
\hline P $7 / 4$ & 0.4457 & 0.3961 & 5 PB $7.1 / 3.8$ & $5.0 \mathrm{P} 7.1 / 4.8$ \\
\hline B $7 / 6$ & .4436 & .3958 & $5 \mathrm{~PB} 7.1 / 3.8$ & $5.0 \mathrm{~B} 7.1 / 4.7$ \\
\hline $10 \mathrm{~B} 7 / 4$ & .4526 & .3954 & $5 \mathrm{~PB} \quad 7.2 / 3.8$ & $9.0 \mathrm{~B} 7.2 / 4.0$ \\
\hline $\mathrm{PB} 7 / 4$ & .4743 & .3885 & $5 \mathrm{~PB} 7.3 / 4.4$ & $4.0 \mathrm{~PB} 7.3 / 4.5$ \\
\hline P 7/ 6 & .4381 & .3765 & $5 \mathrm{~PB} 7.0 / 4.9$ & 5. $5 \mathrm{P} \quad 7.0 / 6.4$ \\
\hline $10 \mathrm{~PB} 7 / 6$ & .4365 & .3737 & $5 \mathrm{~PB} 7.0 / 5.0$ & $10.0 \mathrm{~PB} 7.0 / 5.3$ \\
\hline 10B $7 / 6$ & .4576 & .3709 & $5 \mathrm{~PB} 7.2 / 5.2$ & $9.5 \mathrm{~B} 7.2 / 5.7$ \\
\hline PB $7 / 6$ & .4556 & .3687 & $5 \mathrm{~PB} 7.2 / 5.4$ & 4.0PB $7.2 / 5.6$ \\
\hline $10 \mathrm{YR} 6 / 10$ & .3595 & .8349 & $5 \mathrm{Y} 6.5 / 8.8$ & 10.0 YR $6.5 / 9.8$ \\
\hline 5 YR $6 / 12$ & .3241 & .8401 & $5 \mathrm{Y} 6.2 / 8.6$ & 6.0 YR $6.2 / 11.5$ \\
\hline Y 6/ 8 & .3242 & .8313 & $5 \mathrm{Y} 6.1 / 8.4$ & $\begin{array}{lll}5.5 \mathrm{Y} & 6.2 / 8,3\end{array}$ \\
\hline Y 6/ 8 & .3145 & .8289 & $5 \mathrm{Y} 6.1 / 8.2$ & $5.5 \mathrm{Y} \quad 6.1 / 8.1$ \\
\hline $10 \mathrm{Y} 6 / 6$ & .3278 & .7980 & $5 \mathrm{Y} 6.2 / 7.7$ & $9.5 \mathrm{Y} \quad 6.2 / 7.4$ \\
\hline YR 6/10 & .3350 & .7855 & $5 \mathrm{Y} 6.3 / 7.4$ & $6.0 \mathrm{YR} \quad 6.3 / 9.8$ \\
\hline $\mathrm{Y} 6 / 6$ & .3179 & .7817 & $5 \mathrm{Y} 6.2 / 7.2$ & $5.0 \mathrm{Y} \quad 6.2 / 7.2$ \\
\hline $10 \mathrm{YR} 6 / 8$ & .3457 & .7653 & $5 Y \quad 6.4 / 7.0$ & $9.5 \mathrm{YR} 6.4 / 8.0$ \\
\hline GY 6/ 8 & .3238 & .7457 & $5 Y 6.2 / 6.5$ & 4.0GY $6.2 / 7.2$ \\
\hline GY 6/ 6 & .3200 & .7246 & $5 \mathrm{Y} 6.2 / 5.9$ & 5.0GY $6.2 / 6.7$ \\
\hline YR 6/ 8 & .3274 & .7192 & $5 Y \quad 6.2 / 5.9$ & 5.0 YR $6.2 / 8.3$ \\
\hline $10 \mathrm{YR} 6 / 6$ & .3392 & .7150 & $5 \mathrm{Y} 6.3 / 5.8$ & $9.5 \mathrm{YR} 6.3 / 6.7$ \\
\hline $10 \mathrm{R} 6 / 10$ & .3243 & .7068 & $5 Y \quad 6.2 / 5.6$ & $0.5 \mathrm{YR} \quad 6.2 / 10.1$ \\
\hline $10 \mathrm{GY} 6 / 10$ & .3032 & .7025 & $5 \mathrm{Y} 6.0 / 5.4$ & $9.0 \mathrm{GY} 6.0 / 8.7$ \\
\hline $10 \mathrm{GY} 6 / 8$ & .3224 & .6692 & $5 \mathrm{Y} 6.2 / 4.8$ & $9.0 \mathrm{GY} \quad 6.2 / 7.7$ \\
\hline $10 \mathrm{R} 6 / 8$ & .3345 & .6466 & $5 \mathrm{Y} \quad 6.3 / 4.3$ & $0.5 \mathrm{YR} 6.3 / 8.2$ \\
\hline $10 \mathrm{Y} 6 / 4$ & .3291 & .6411 & $5 Y \quad 6.2 / 4.2$ & $10.0 \mathrm{Y} 6.2 / 4.1$ \\
\hline YR 6/ 6 & .3221 & .6410 & $5 \mathrm{Y} 6.2 / 4.2$ & 4.5YR $6.2 / 6.0$ \\
\hline $\mathrm{Y} 6 / 4$ & .3100 & .6385 & $5 Y \quad 6.1 / 4.1$ & 4. $5 \mathrm{Y} 6.1 / 4.0$ \\
\hline $10 \mathrm{GY} 6 / 6$ & .3168 & .6299 & $5 Y 6.1 / 3.9$ & $10.0 \mathrm{GY} 6.1 / 6.7$ \\
\hline $10 \mathrm{YR} 6 ! 4$ & .3149 & .6173 & $5 \mathrm{Y} 6.1 / 3.6$ & $0.5 \mathrm{Y} \quad 6.1 / 4.1$ \\
\hline GY 6/ 4 & .3217 & .6151 & $5 \mathrm{Y} 6.2 / 3.6$ & $5.0 \mathrm{GY} \quad 6.2 / 4.0$ \\
\hline $10 \mathrm{R} 6 / 6$ & .3384 & .5995 & $5 \mathrm{Y} \quad 6.3 / 3.3$ & $0.5 \mathrm{YR} 6.3 / 6.5$ \\
\hline YR 6/ 4 & .3113 & .5751 & $5 \mathrm{Y} \quad 6.2 / 2.6$ & 4.0YR $6.1 / 4.0$ \\
\hline R $6 / 10$ & .3236 & .5628 & $5 \mathrm{Y} 6.2 / 2.4$ & 4. $0 \mathrm{R} 6.2 / 9.7$ \\
\hline $10 \mathrm{GY} 6 / 4$ & .3101 & .5598 & $5 \mathrm{Y} 6.1 / 2.3$ & $0.5 \mathrm{G} \quad 6.1 / 4.4$ \\
\hline R 6/ 8 & .3236 & .5436 & $5 \mathrm{Y} 6.2 / 1.9$ & 4. $0 \mathrm{R} \quad 6.2 / 8.1$ \\
\hline $10 \mathrm{R} 6 / 4$ & .3262 & .5432 & $5 \mathrm{Y} 6.2 / 1.9$ & $9.0 \mathrm{R} 6.2 / 4.2$ \\
\hline Y 6/ 2 & .3211 & .5317 & $5 \mathrm{Y} 6.2 / 1.7$ & 4. 0 Y $6.2 / 1.7$ \\
\hline GY 6/2 & .3245 & .5239 & $5 Y 6.2 / 1.7$ & $5.0 \mathrm{GY} 6.2 / 1.7$ \\
\hline G $6 / 6$ & .3040 & .5237 & $5 \mathrm{Y} 6.0 / 1.5$ & $6.0 \mathrm{G} 6.0 / 7.4$ \\
\hline R 6/ 6 & .3308 & .5200 & $5 \mathrm{Y} 6.2 / 1.4$ & $3.5 \mathrm{R} 6.2 / 6.3$ \\
\hline YR 6/ 2 & .3124 & .5155 & $5 Y \quad 6.1 / 1.3$ & 4. $5 \mathrm{YR} 6.1 / 2.0$ \\
\hline $\mathrm{R} 6 / 4$ & .3313 & .5065 & $5 \mathrm{Y} 6.3 / 1.1$ & 4. $5 \mathrm{R} 6.3 / 4.5$ \\
\hline G $6 / 4$ & .3151 & .5018 & $5 \mathrm{Y} 6.1 / 1.0$ & $6.0 \mathrm{G} 6.1 / 4.6$ \\
\hline $10 \mathrm{RP} 6 / 10$ & .3210 & .4964 & $5 \mathrm{Y} 6.2 / 0.9$ & 9.5RP $6.2 / 8.8$ \\
\hline 10RP 6/ 8 & .3248 & .4875 & $5 Y \quad 6.2 / 0.7$ & 9.0RP $6.2 / 7.0$ \\
\hline $10 \mathrm{G} 6 / 2$ & .3032 & .4836 & $5 \mathrm{Y} 6.0 / 0.6$ & $6.0 \mathrm{G} 6.0 / 2.4$ \\
\hline $\mathrm{R} 6 / 2$ & .3205 & .4806 & $5 \mathrm{Y} 6.2 / 0.6$ & 4. $0 \mathrm{R} 6.2 / 2.3$ \\
\hline $10 \mathrm{RP} 6 / 6$ & .3240 & .4792 & $5 \mathrm{Y} \quad 6.2 / 0.6$ & $9.0 \mathrm{RP} \quad 6.2 / 5.5$ \\
\hline $10 \mathrm{G} 6 / 6$ & .3538 & .4756 & $5 \mathrm{Y} 6.4 / 0.5$ & 1. $0 \mathrm{BG} 6.4 / 6.0$ \\
\hline 10RP 6/ 4 & .3239 & .4700 & $5 \mathrm{Y} \quad 6.2 / 0.5$ & 8.5RP $6.2 / 4.0$ \\
\hline G $6 / 4$ & .3458 & .4677 & $5 \mathrm{Y} \quad 6.4 / 0.3$ & $2.0 \mathrm{BG} 6.4 / 4.5$ \\
\hline N 6/ & .3032 & .4586 & N 6.0/ & N $6.0 /$ \\
\hline $5 \mathrm{RP} 6 / 2$ & .3169 & .4548 & $5 \mathrm{~PB} 6.1 / 0.2$ & 4.0RP $6.1 / 2.2$ \\
\hline $5 \mathrm{BG} 6 / 2$ & .3429 & .4524 & $5 \mathrm{~PB} 6.4 / 0.2$ & 5.0BG $6.4 / 2.4$ \\
\hline $5 \mathrm{RP} 6 / 4$ & .3454 & .4504 & $5 \mathrm{~PB} 6.4 / 0.3$ & 4.5RP $6.4 / 4.2$ \\
\hline $5 \mathrm{RP} 6 / 6$ & .3345 & .4503 & $5 \mathrm{~PB} 6.3 / 0.3$ & 4. $5 \mathrm{RP} 6.3 / 5.6$ \\
\hline $5 \mathrm{RP} 6 / 8$ & .3349 & .4438 & $5 \mathrm{~PB} \quad 6.3 / 0.7$ & 5.0RP $6.3 / 7.3$ \\
\hline
\end{tabular}

TABLE 4. Deuteranopic arrangement-Continued

\begin{tabular}{|c|c|c|c|c|}
\hline \multirow{2}{*}{$\begin{array}{l}\text { Munsell } \\
\text { book nota- } \\
\text { tion }\end{array}$} & \multirow{2}{*}{$\begin{array}{c}\text { Deuteran- } \\
\text { opic re- } \\
\text { flectance, } \\
W_{d}=Y\end{array}$} & \multirow{2}{*}{$\begin{array}{c}\text { Deuteran- } \\
\text { opic chro- } \\
\text { maticity } \\
\text { coordinate, } \\
w_{d}\end{array}$} & \multicolumn{2}{|c|}{ Munsell renotations } \\
\hline & & & Deuteranopic & Normal \\
\hline $\mathrm{BG} 6 / 4$ & 0.3425 & 0.4431 & $5 \mathrm{~PB} 6.4 / 0.8$ & $5.5 \mathrm{BG} 6.4 / 4.1$ \\
\hline BG $6 / 6$ & .3473 & .4379 & $5 \mathrm{~PB} 6.4 / 1.1$ & $5.5 \mathrm{BG} 6.4 / 5.2$ \\
\hline RP $6 / 10$ & .3389 & .4358 & $5 \mathrm{~PB} 6.3 / 1.1$ & 4.5 RP $6.3 / 8.5$ \\
\hline $10 \mathrm{P} 6 / 4$ & .3238 & .4176 & $5 \mathrm{~PB} 6.2 / 2.2$ & 10.0P $6.2 / 4.6$ \\
\hline $10 \mathrm{BG} 6 / 4$ & .3316 & .4163 & $5 \mathrm{~PB} 6.3 / 2.3$ & $10.0 \mathrm{BG} 6 / 34.1$ \\
\hline P 6/ 2 & .3238 & .4126 & $5 \mathrm{~PB} 6.2 / 2.5$ & 4. $5 \mathrm{P} \quad 6.2 / 3.0$ \\
\hline B $6 / 2$ & .3234 & .4109 & $5 \mathrm{~PB} 6.2 / 2.6$ & 7. $5 \mathrm{~B} \quad 6.2 / 3.0$ \\
\hline $\mathrm{PB} 6 / 2$ & .3432 & .4007 & $5 \mathrm{~PB} 6.4 / 3.1$ & 5. 0 PB $6.4 / 3.2$ \\
\hline $10 \mathrm{P} 6 / 6$ & .3307 & .4005 & $5 \mathrm{~PB} 6.2 / 3.1$ & 9. $5 \mathrm{P} 6.2 / 6.2$ \\
\hline $10 \mathrm{BG} 6 / 6$ & .3375 & .3995 & $5 \mathrm{~PB} 6.3 / 3.2$ & $10.0 \mathrm{BG} 6.3 / 5.8$ \\
\hline B $6 / 4$ & .3250 & .3858 & $5 \mathrm{~PB} 6.2 / 3.9$ & $6.5 \mathrm{~B} 6.2 / 4.5$ \\
\hline $\mathrm{P} 6 / 4$ & .3301 & .3840 & $5 \mathrm{~PB} 6.2 / 4.0$ & 4. $0 \mathrm{P} 6.2 / 4.9$ \\
\hline $10 \mathrm{P} 6 / 8$ & .3245 & .3820 & $5 \mathrm{~PB} 6.2 / 4.1$ & 9.0P 6.2/ 8.0 \\
\hline $10 \mathrm{~B} 6 / 4$ & .3415 & .3777 & $5 \mathrm{~PB} 6.3 / 4.5$ & $10.0 \mathrm{~B} 6.3 / 4.6$ \\
\hline PB 6/ 4 & .3589 & .3753 & $5 \mathrm{~PB} 6.5 / 4.6$ & $5.0 \mathrm{~PB} 6.5 / 4.7$ \\
\hline $10 \mathrm{~PB} 6 / 4$ & .3394 & .3729 & $5 \mathrm{~PB} 6.3 / 4.6$ & $0.5 \mathrm{P} \quad 6.3 / 4.8$ \\
\hline B $6 / 6$ & .3128 & .3586 & $5 \mathrm{~PB} 6.1 / 5.3$ & $5.5 \mathrm{~B} 6.1 / 6.4$ \\
\hline $10 \mathrm{~B} 6 / 6$ & .3320 & .3557 & $5 \mathrm{~PB} 6.3 / 5.4$ & $9.0 \mathrm{~B} 6.3 / 6.3$ \\
\hline $\mathrm{P} 6 / 6$ & .3179 & .3545 & $5 \mathrm{~PB} 6.2 / 5.4$ & 4. $5 \mathrm{P} 6.2 / 7.1$ \\
\hline $10 \mathrm{~PB} 6 / 6$ & .3161 & .3455 & $5 \mathrm{~PB} 6.1 / 5.9$ & 10.0PB $6.1 / 6.3$ \\
\hline PB 6/ 6 & .3404 & .3447 & $5 \mathrm{~PB} 6.3 / 6.1$ & $5.0 \mathrm{~PB} 6.3 / 6.2$ \\
\hline P 6/ 8 & .3157 & .3316 & $5 \mathrm{~PB} 6.1 / 6.7$ & 5. $0 \mathrm{P}$ 6.1/ 9.1 \\
\hline PB $6 / 8$ & .3329 & .3260 & $5 \mathrm{~PB} 6.3 / 7.4$ & $5.0 \mathrm{~PB} 6.3 / 7.4$ \\
\hline $10 \mathrm{~PB} 6 / 8$ & .3200 & .3210 & $5 \mathrm{PY} 6.2 / 7.4$ & $0.5 \mathrm{P} \quad 6.2 / 8.0$ \\
\hline $5 \mathrm{YR} 5 / 10$ & .2056 & .8101 & $5 Y \quad 5.1 / 6.9$ & $5.5 \mathrm{YR} 5.1 / 9.0$ \\
\hline $10 \mathrm{YR} 5 / 8$ & .2099 & .8054 & $5 Y 5.1 / 6.8$ & $0.5 \mathrm{Y} 5.1 / 7.6$ \\
\hline $\mathrm{Y} 5 / 6$ & .1984 & .7817 & $5 \mathrm{Y} 5.0 / 6.1$ & $6.0 \mathrm{Y} 5.0 / 6.0$ \\
\hline GY 5/ 8 & .2100 & .7743 & $5 \mathrm{Y} 5.1 / 6.1$ & 5.0GY $5.1 / 7.1$ \\
\hline YR 5/ 8 & 2081 & .7537 & $5 \mathrm{Y} 5.1 / 5.7$ & $5.5 \mathrm{YR} 5.1 / 7.7$ \\
\hline $10 \mathrm{Y} 5 / 6$ & .2250 & .7535 & $5 \mathrm{Y} 5.3 / 5.8$ & $10.0 \mathrm{Y} 5.3 / 5.8$ \\
\hline 10R $5 / 10$ & .1977 & .7446 & $5 Y 5.0 / 5.7$ & $10.0 \mathrm{R} 5.0 / 9.9$ \\
\hline 10 YR 5/ 6 & .2121 & .7132 & $5 \mathrm{Y} 5.2 / 5.0$ & 10.0YR $5.2 / 5.7$ \\
\hline $10 \mathrm{R} 5 / 8$ & .2175 & .6976 & $5 Y 5.2 / 4.7$ & 0.5 YR $5.2 / 8.7$ \\
\hline GY 5/ 6 & .2066 & .6931 & $5 Y 5.1 / 4.6$ & 5.0GY 5.1/5.4 \\
\hline $10 \mathrm{GY} 5 / 8$ & .1910 & .6963 & $5 Y \quad 4.9 / 4.4$ & $9.5 \mathrm{GY} 4.9 / 7.8$ \\
\hline YR 5/ 6 & .1981 & .6895 & $5 Y \quad 5.0 / 4.4$ & 5.0 YR $5.0 / 6.2$ \\
\hline $10 \mathrm{Y} 5 / 4$ & .2224 & .6776 & $5 Y \quad 5.3 / 4.4$ & $0.5 \mathrm{GY} 5.3 / 4.4$ \\
\hline Y 5/ 4 & .1987 & .6657 & $5 \mathrm{Y} 5.0 / 4.0$ & $5.0 Y 5.0 / 3.9$ \\
\hline $10 \mathrm{GY} 5 / 6$ & .2141 & .6645 & $5 \mathrm{Y} 5.2 / 4.0$ & 9.5GY 5.2/ 7.1 \\
\hline R $5 / 12$ & .1938 & .6608 & $5 \mathrm{Y} 5.0 / 3.8$ & 5. $5 \mathrm{R} 5.0 / 12.8$ \\
\hline $10 \mathrm{YR} 5 / 4$ & .2048 & .6554 & $5 Y 5.1 / 3.8$ & 10.0YR 5.1/4. 4 \\
\hline $10 \mathrm{R} 5 / 6$ & .2073 & .6472 & $5 Y 5.1 / 3.6$ & 10.0R 5.1/ 7.1 \\
\hline GY 5/ 4 & .2070 & .6338 & $5 Y \quad 5.1 / 3.4$ & 5.0GY $5.1 / 4.1$ \\
\hline R $5 / 10$ & .2128 & .6224 & $5 \mathrm{Y} \quad 5.2 / 3.3$ & 5. GR 5. 2/10.9 \\
\hline YR $5 / 4$ & .1905 & .6173 & $5 Y \quad 4.9 / 3.1$ & 4. 5 YR $4.9 / 4.6$ \\
\hline R 5/ 8 & .1963 & .5988 & $5 Y 5.0 / 2.7$ & 4. $5 \mathrm{R} 5.0 / 10.0$ \\
\hline $10 \mathrm{R} 5 / 4$ & .2028 & .5774 & $5 Y 5.1 / 2.3$ & 9. $5 \mathrm{R} 5.1 / 4.9$ \\
\hline $10 \mathrm{GY} 5 / 4$ & .2025 & .5766 & $5 Y 5.0 / 2.3$ & $10.0 \mathrm{GY} 5.0 / 4.3$ \\
\hline R $5 / 6$ & 1998 & .5594 & $5 \mathrm{Y} 5.0 / 2.0$ & 4. $0 \mathrm{R} 5.0 / 8.0$ \\
\hline G $5 / 8$ & .1875 & .5521 & $5 Y 4.9 / 1.8$ & 5. $0 \mathrm{G} 4.9 / 8.2$ \\
\hline Y 5/ 2 & .2117 & .5412 & $5 \mathrm{Y} 5.2 / 1.7$ & $4.5 \mathrm{Y} 5.2 / 1.7$ \\
\hline GY $5 / 2$ & .2097 & .5376 & $5 Y \quad 5.1 / 1.6$ & 5.0GY 5.1/ 2.0 \\
\hline G $5 / 6$ & .1869 & .5348 & $5 \mathrm{Y} 4.9 / 1.5$ & 5. $0 \mathrm{G} 4.9 / 6.6$ \\
\hline R 5/ 4 & .1915 & .5275 & $5 \mathrm{Y} 4.9 / 1.4$ & 4.0R $4.9 / 5.3$ \\
\hline YR $5 / 2$ & .1927 & .5234 & $5 \mathrm{Y} 4.9 / 1.3$ & 4.0YR 4.9/2.1 \\
\hline G $5 / 4$ & .1856 & .5195 & $5 Y 4.9 / 1.2$ & $5.0 \mathrm{G} 4.9 / 4.8$ \\
\hline
\end{tabular}


TABle 4. Deuteranopic arrangement-Continued

\begin{tabular}{|c|c|c|c|c|}
\hline \multirow{2}{*}{$\begin{array}{l}\text { Munsell } \\
\text { book nota- } \\
\text { tion }\end{array}$} & \multirow{2}{*}{$\begin{array}{c}\text { Deuteran- } \\
\text { opic re- } \\
\text { flectance, } \\
W_{d}=Y\end{array}$} & \multirow{2}{*}{$\begin{array}{c}\text { Deuteran- } \\
\text { opic chro- } \\
\text { maticity } \\
\text { coordinate, } \\
w_{d}\end{array}$} & \multicolumn{2}{|c|}{ Munsell renotations } \\
\hline & & & Deuteranopic & Normal \\
\hline $10 \mathrm{RP} 5 / 10$ & 0.2180 & 0.5156 & $5 Y \quad 5.2 / 1.2$ & 10.0RP $5.2 / 10.3$ \\
\hline $10 \mathrm{RP} 5 / 8$ & .2111 & .5086 & $5 \mathrm{Y} 5.2 / 1.0$ & 10.0RP 5. $2 / 8.6$ \\
\hline $10 \mathrm{RP} \mathrm{5/} 6$ & .2191 & .4932 & $5 Y \quad 5.2 / 0.7$ & 10.0RP 5.2/6.6 \\
\hline R 5/2 & .1954 & .4891 & $5 Y \quad 5.0 / 0.6$ & 4. $0 \mathrm{R} 5.0 / 2.8$ \\
\hline G $5 / 2$ & .1888 & .4835 & $5 \mathrm{Y} 4.9 / 0.5$ & $6.5 \mathrm{G} \quad 4.9 / 2.4$ \\
\hline $10 \mathrm{RP} 5 / 4$ & .2260 & .4814 & $5 Y 5.3 / 0.5$ & 10.0RP $5.3 / 4.2$ \\
\hline $10 \mathrm{G} 5 / 6$ & .2013 & .4767 & $5 Y \quad 5.0 / 0.4$ & 1.0BG $5.0 / 7.0$ \\
\hline $10 \mathrm{G} 5 / 4$ & .2047 & .4707 & $5 \mathrm{Y} 5.1 / 0.2$ & $0.5 \mathrm{BG} 5.1 / 4.6$ \\
\hline RP 5/ 2 & .2138 & .4552 & $5 \mathrm{~PB} 5.2 / 0.1$ & 5.0RP 5.2/ 2.3 \\
\hline N 5/ & .1906 & .4540 & $5 \mathrm{~PB} 4.9 / 0.2$ & 5. $0 \mathrm{~PB} 4.9 / 0.1$ \\
\hline BG $5 / 2$ & .2077 & .4501 & $5 \mathrm{~PB} 5.1 / 0.3$ & 4. $5 \mathrm{BG} 5.1 / 2.5$ \\
\hline RP 5/ 4 & .2110 & .4458 & $5 \mathrm{~PB} 52 / 04$ & 5.0RP $5.2 / 4.3$ \\
\hline $\mathrm{RP} 5 / 6$ & .2099 & .4410 & $5 \mathrm{~PB} 5.1 / 0.7$ & 4. $5 \mathrm{RP} 5.1 / 6.2$ \\
\hline $5 \mathrm{RP} 5 / 10$ & .2301 & .4395 & $5 \mathrm{~PB} 5.3 / 0.8$ & 5.5RP $5.3 / 9.7$ \\
\hline RP 5/ 8 & .2206 & .4356 & $5 \mathrm{~PB} 5.2 / 1.0$ & 4. $5 \mathrm{RP} 5.2 / 8.0$ \\
\hline BG $5 / 4$ & .2112 & .4323 & $5 \mathrm{~PB} 5.2 / 1.1$ & 5. $5 \mathrm{BG} 5.2 / 4.4$ \\
\hline $\mathrm{BG} \mathrm{5/6}$ & .2097 & .4250 & $5 \mathrm{~PB} 5.1 / 1.4$ & 5.0BG $5.1 / 6.4$ \\
\hline P 5/ 2 & .1959 & .4101 & $5 \mathrm{~PB} 5.0 / 2.0$ & $5.0 \mathrm{P} \quad 5.0 / 2.5$ \\
\hline $10 \mathrm{P} 5 / 4$ & .2143 & .4089 & $5 \mathrm{~PB} 5.2 / 2.2$ & 1.0RP $5.2 / 4.9$ \\
\hline B $5 / 2$ & .1917 & .4037 & $5 \mathrm{~PB} 4.9 / 2.2$ & 6. $5 \mathrm{~B} 4.9 / 2.6$ \\
\hline $10 \mathrm{BG} 5 / 4$ & .2062 & .3998 & $5 \mathrm{~PB} 5.1 / 2.5$ & $0.5 \mathrm{~B} \quad 5.1 / 4.4$ \\
\hline $10 \mathrm{P} 5 / 6$ & .2129 & .3922 & 5 PB 5. $2 / 2.9$ & 1.0RP $5.2 / 6.7$ \\
\hline PB 5/ 2 & .2087 & .3891 & $5 \mathrm{~PB} 5.1 / 3.0$ & 5.0PB $5.1 / 3.1$ \\
\hline $10 \mathrm{P} 5 / 8$ & .2174 & .3737 & 5 PB 5. $2 / 3.8$ & 1.0RP 5. $2 / 8.6$ \\
\hline P 5/ 4 & .1934 & .3697 & 5 PB $5.0 / 3.8$ & 4. $5 \mathrm{P} 5.0 / 4.7$ \\
\hline $10 \mathrm{BG} 5 / 6$ & .2160 & .3687 & $5 \mathrm{~PB} 5.2 / 4.0$ & 1.0 B $5.2 / 6.5$ \\
\hline B $5 / 4$ & .1959 & .3618 & 5 PB $5.0 / 4.2$ & $5.5 \mathrm{~B} 5.0 / 5.1$ \\
\hline $10 \mathrm{~B} 5 / 4$ & .2134 & .3616 & 5 PB 5.2/ 4.3 & $10.0 \mathrm{~B} 5.2 / 4.6$ \\
\hline PB 5/ 4 & .2170 & .3599 & 5 PB 5.2/4.5 & 4. 5PB 5. $2 / 4.4$ \\
\hline $10 \mathrm{P} 5 / 10$ & .2226 & .3576 & $5 \mathrm{~PB} 5.3 / 4.6$ & 0.5 RP $5.3 / 10.4$ \\
\hline $10 \mathrm{~PB} 5 / 4$ & .2180 & .3528 & 5 PB $5.2 / 4.7$ & 10.0PB 5. $2 / 5.0$ \\
\hline P 5/ 6 & .1965 & .3424 & $5 \mathrm{~PB} 5.0 / 5.1$ & 5.0P 5.0/6.8 \\
\hline B $5 / 6$ & .1940 & .3333 & 5 PB $5.0 / 5.5$ & 5.0 B $5.0 / 6.8$ \\
\hline $10 \mathrm{~B} 5 / 6$ & .2132 & .3292 & 5 PB 5. $2 / 5.8$ & 9. 5 B 5. $2 / 6.3$ \\
\hline PB 5/ 6 & .2241 & .3299 & 5 PB $5.3 / 6.1$ & 4.0PB 5.3/6.2 \\
\hline 10 PB 5/ 6 & .2149 & .3223 & 5 PB $5.2 / 6.3$ & 10.0РB 5. $2 / 6.8$ \\
\hline P 5/ 8 & .2107 & .3154 & 5 PB $5.1 / 6.6$ & 5.0P 5.1/ 8.9 \\
\hline PB 5/ 8 & .2345 & .3036 & 5 PB $5.4 / 7.5$ & 4.0РB $5.4 / 7.9$ \\
\hline $10 \mathrm{~PB} 5 / 8$ & .2126 & .2938 & 5 PB 5.2/ 7.8 & 10.0PB 5. $2 / 8.4$ \\
\hline P $5 / 10$ & .2039 & .2893 & $5 \mathrm{~PB} 5.1 / 8.1$ & 5. OP 5. $1 / 10.9$ \\
\hline PB $5 / 10$ & .2263 & .2871 & 5 PB $5.3 / 8.4$ & 3. 5PB 5. $3 / 8.6$ \\
\hline $10 \mathrm{~PB} 5 / 10$ & .2014 & .2757 & 5 PB $5.0 / 8.8$ & 10.0PB $5.0 / 9.6$ \\
\hline $5 \mathrm{YR} 4 / 8$ & .1354 & .7407 & $5 \mathrm{Y} 4.2 / 5.8$ & 6.0 YR $4.2 / 6.3$ \\
\hline $10 \mathrm{R} 4 / 10$ & .1493 & .7234 & $5 Y 4.4 / 4.6$ & $9.5 \mathrm{R} 4.4 / 9.0$ \\
\hline $10 \mathrm{R} \mathrm{4/8}$ & .1428 & .7112 & $5 \mathrm{Y} 4.3 / 4.3$ & 10.0R $4.3 / 8.4$ \\
\hline $10 \mathrm{YR} 4 / 4$ & .1318 & .7063 & $5 \mathrm{Y} 4.2 / 4.2$ & 10.0YR 4. $2 / 4.6$ \\
\hline R $4 / 14$ & .1334 & .7081 & $5 \mathrm{Y} 4.2 / 4.2$ & 5. $5 \mathrm{R} 4.2 / 13.2$ \\
\hline R $4 / 14$ & .1335 & .7034 & $5 \mathrm{Y} 4.2 / 4.2$ & 5. 5 R $4.2 / 13.2$ \\
\hline YR 4/ 6 & .1445 & .7032 & $5 \mathrm{Y} 4.4 / 4.2$ & 5.0 YR 4. 4/ 5.8 \\
\hline Y 4/ 4 & .1243 & .6871 & $5 Y 4.1 / 3.7$ & $5.0 \mathrm{Y} 4.1 / 3.6$ \\
\hline R 4/12 & .1360 & .6831 & $5 Y 4.2 / 3.8$ & 5. $0 \mathrm{R}$ 4. $2 / 12.6$ \\
\hline $10 \mathrm{Y} 4 / 4$ & .1385 & .6733 & $5 Y 4.3 / 3.7$ & $10.0 \mathrm{Y} 4.3 / 3.7$ \\
\hline $10 \mathrm{R} 4 / 6$ & .1424 & .6726 & $5 Y 4.3 / 3.7$ & $10.0 \mathrm{R} 4.3 / 7.3$ \\
\hline GY 4/ 6 & .1249 & .6661 & $5 \mathrm{Y} 4.1 / 3.4$ & 5.0GY 4.1/ 4.0 \\
\hline YR 4/ 4 & .1416 & .6556 & $5 Y 4.3 / 3.4$ & 5. 0 YR $4.3 / 4.6$ \\
\hline R 4/10 & .1303 & .6307 & $5 Y 4.2 / 3.0$ & 4. $5 \mathrm{R} 4.2 / 10.8$ \\
\hline
\end{tabular}

TABLE 4. Deuteranopic arrangement-Continued

\begin{tabular}{|c|c|c|c|c|}
\hline \multirow{2}{*}{$\begin{array}{l}\text { Munsell } \\
\text { book nota- } \\
\text { tion }\end{array}$} & \multirow{2}{*}{$\begin{array}{c}\text { Deuteran- } \\
\text { opic re- } \\
\text { flectance, } \\
W_{d}=Y\end{array}$} & \multirow{2}{*}{$\begin{array}{c}\text { Deuteran- } \\
\text { opic chro- } \\
\text { maticity } \\
\text { coordinate, } \\
w_{d}\end{array}$} & \multicolumn{2}{|c|}{ Munsell renotations } \\
\hline & & & Deuteranopic & Normal \\
\hline $10 \mathrm{GY} 4 / 6$ & 0.1355 & 0.6279 & $5 \mathrm{Y} 4.2 / 2.9$ & $10.0 \mathrm{GY} 4.2 / 5.4$ \\
\hline GY 4/ 4 & .1313 & .6173 & $5 \mathrm{Y} 4.2 / 2.7$ & $5.0 \mathrm{GY} 4.2 / 3.2$ \\
\hline $\mathrm{R} \mathrm{4/8}$ & .1306 & .6111 & $5 \mathrm{Y} 4.2 / 2.6$ & 4. $5 \mathrm{R} 4.2 / 9.4$ \\
\hline $10 \mathrm{R} \mathrm{4/4}$ & .1348 & .6007 & $5 Y 4.2 / 2.5$ & $0.5 \mathrm{YR} 4.2 / 4.7$ \\
\hline $10 \mathrm{GY} 4 / 4$ & .1337 & .5716 & $5 \mathrm{Y} 4.2 / 2.0$ & 1. $0 \mathrm{G} 4.2 / 4.1$ \\
\hline R 4/ 6 & .1247 & .5663 & $5 Y 4.1 / 1.9$ & 4. $5 \mathrm{R} 4.1 / 6.8$ \\
\hline $\mathrm{Y} 4 / 2$ & .1419 & .5534 & $5 \mathrm{Y} 4.3 / 1.7$ & $5.5 \mathrm{Y} 4.3 / 1.7$ \\
\hline YR 4/ 2 & .1268 & .5484 & $5 \mathrm{Y} 4.1 / 1.5$ & $4.0 \mathrm{YR} 4.1 / 2.3$ \\
\hline GY 4/ 2 & .1357 & .5357 & $5 Y 4.2 / 1.4$ & $5.5 \mathrm{GY} 4.2 / 1.7$ \\
\hline R 4/ 4 & .1236 & .5257 & $5 \mathrm{Y} 4.1 / 1.2$ & $3.5 \mathrm{R} 4.1 / 4.7$ \\
\hline G $4 / 4$ & .1291 & .5218 & $5 \mathrm{Y} 4.1 / 1.1$ & $5.0 \mathrm{G} 4.1 / 4.9$ \\
\hline $10 \mathrm{RP} 4 / 10$ & .1359 & .5024 & $5 \mathrm{Y} 4.2 / 0.8$ & 9.5RP $4.2 / 9.5$ \\
\hline R 4/ 2 & .1280 & .4963 & $5 Y 4.1 / 0.7$ & . 4. $0 \mathrm{R} 4.1 / 2.6$ \\
\hline 10RP 4/ 6 & .1262 & .4903 & $5 Y 4.1 / 0.6$ & $9.5 \mathrm{RP} 4.1 / 6.4$ \\
\hline G $4 / 2$ & .1252 & .4884 & $5 \mathrm{Y} 4.1 / 0.6$ & $6.0 \mathrm{G} 4.1 / 2.4$ \\
\hline $10 \mathrm{RP} 4 / 8$ & .1332 & .4876 & $5 Y \quad 4.2 / 0.5$ & 9.0RP $4.2 / 8.2$ \\
\hline $10 \mathrm{RP} 4 / 4$ & .1277 & .4754 & $5 \mathrm{Y} 4.1 / 0.3$ & 9.0RP 4.1/4.1 \\
\hline $10 \div 4 / 4$ & .1233 & .4731 & $5 Y \quad 4.1 / 0.3$ & $0.5 \mathrm{BG} 4.1 / 4.5$ \\
\hline N 4/ & .1209 & .4526 & $5 \mathrm{~PB} 4.0 / 0.1$ & $\begin{array}{llll}5.0 \mathrm{~PB} & 4.0 / 0.1\end{array}$ \\
\hline $\mathrm{RP} 4 / 2$ & .1445 & .4474 & $5 \mathrm{~PB} 4.4 / 0.3$ & 4.0RP $4.4 / 2.1$ \\
\hline BG 4/ 2 & .1382 & .4424 & $5 \mathrm{~PB} \quad 4.3 / 0.4$ & 5. $5 \mathrm{BG} 4.3 / 2.5$ \\
\hline $\mathrm{RP} 4 / 4$ & .1418 & .4332 & $5 \mathrm{~PB} \quad 4.3 / 0.8$ & 4.0RP $4.3 / 4.4$ \\
\hline $\mathrm{BG} 4 / 4$ & .1413 & .4296 & $5 \mathrm{~PB} \quad 4.3 / 0.9$ & $5.5 \mathrm{BG} 4.3 / 4.0$ \\
\hline RP 4/ 6 & .1406 & .4230 & $5 \mathrm{~PB} \quad 4.3 / 1.1$ & 4.5 RP $4.3 / 6.4$ \\
\hline RP 4/ 8 & .1341 & .4172 & $5 \mathrm{~PB} \quad 4.2 / 1.3$ & 4.5RP $4.2 / 7.9$ \\
\hline RP $4 / 10$ & .1359 & .4127 & $5 \mathrm{~PB} 4.2 / 1.5$ & 4.5R P $4.2 / 9.4$ \\
\hline $\mathrm{BG} 4 / 6$ & .1354 & .4114 & $5 \mathrm{~PB} 4.2 / 1.5$ & $5.5 \mathrm{BG} 4.2 / 6.0$ \\
\hline $5 \mathrm{RP} 4 / 12$ & .1396 & .4076 & $5 \mathrm{~PB} 4.3 / 1.7$ & 5.0RP $4.3 / 10.7$ \\
\hline $5 \mathrm{P} 4 / 2$ & .1248 & .4023 & $5 \mathrm{~PB} 4.1 / 1.8$ & 5. $0 \mathrm{P} 4.1 / 2.2$ \\
\hline $5 \mathrm{~B} 4 / 2$ & .1374 & .4005 & $5 \mathrm{~PB} 4.3 / 1.9$ & $5.0 \mathrm{~B} \quad 4.3 / 2.7$ \\
\hline $10 \mathrm{BG} 4 / 4$ & .1290 & .4002 & $5 \mathrm{~PB} 4.1 / 1.9$ & $9.5 \mathrm{BG} 4.1 / 4.0$ \\
\hline $10 \mathrm{P} 4 / 4$ & .1428 & .3969 & $5 \mathrm{~PB} 4.3 / 2.1$ & $0.5 \mathrm{RP} 4.3 / 4.5$ \\
\hline PB 4/ 2 & .1313 & .3388 & $5 \mathrm{~PB} 4.2 / 2.4$ & $5.0 \mathrm{~PB} 4.2 / 2.5$ \\
\hline $10 \mathrm{BG} 4 / 6$ & .1349 & .3721 & $5 \mathrm{~PB} 4.2 / 3.0$ & $10.0 \mathrm{BG} 4.2 / 5.7$ \\
\hline $10 \mathrm{P} 4 / 6$ & .1407 & .3718 & $5 \mathrm{~PB} 4.3 / 3.1$ & $0.5 \mathrm{RP} 4.3 / 6.6$ \\
\hline P 4/ 4 & .1289 & .3653 & $5 \mathrm{~PB} 4.1 / 3.2$ & 5. $0 \mathrm{P} 4.1 / 4.2$ \\
\hline B $4 / 4$ & .1447 & .3557 & $5 \mathrm{~PB} 4.4 / 3.7$ & $60 \mathrm{~B} \quad 4.4 / 4.6$ \\
\hline $10 \mathrm{~B} 4 / 4$ & .1299 & .3527 & $5 \mathrm{~PB} 4.2 / 3.7$ & $0.5 \mathrm{~PB} \quad 4.2 / 4.1$ \\
\hline $10 \mathrm{P} 4 / 8$ & .1406 & .3519 & $5 \mathrm{~PB} 4.3 / 3.9$ & $0.5 \mathrm{RP} 4.3 / 8.5$ \\
\hline PB 4/ 4 & .1355 & .3441 & $5 \mathrm{~PB} 4.2 / 4.2$ & 5. 0 PB $4.2 / 4.3$ \\
\hline $10 \mathrm{~PB} 4 / 4$ & .1288 & .3377 & $5 \mathrm{~PB} 4.1 / 4.4$ & 10. 0 PB 4.1/4.6 \\
\hline P 4/ 6 & .1240 & .3313 & $5 \mathrm{~PB} 4.1 / 4.6$ & 5. $0 \mathrm{P} 4.1 / 6.1$ \\
\hline $10 \mathrm{P} 4 / 10$ & .1331 & .3291 & $5 \mathrm{~PB} 4.2 / 4.8$ & $0.5 \mathrm{RP} 4.2 / 10.4$ \\
\hline B 4/ 6 & .1287 & .3144 & $5 \mathrm{~PB} 4.1 / 5.4$ & 5. $5 \mathrm{~B} \quad 4.1 / 6.4$ \\
\hline PB 4/ 6 & .1364 & .3071 & $5 \mathrm{~PB} 4.2 / 5.9$ & 4. $5 \mathrm{~PB} 4.2 / 6.0$ \\
\hline P 4/ 8 & .1278 & .3020 & $5 \mathrm{~PB} 4.1 / 5.9$ & 5. $0 \mathrm{P} 4.1 / 8.2$ \\
\hline $10 \mathrm{~B} 4 / 6$ & .1316 & .3014 & $5 \mathrm{~PB} \quad 4.2 / 6.0$ & $0.5 \mathrm{~PB} 4.2 / 6.3$ \\
\hline B 4/ 8 & .1172 & .2991 & $5 \mathrm{~PB} 4.0 / 5.9$ & 6. $5 \mathrm{~B} \quad 4.0 / 6.9$ \\
\hline $10 \mathrm{~PB} 4 / 6$ & .1321 & .2985 & $5 \mathrm{~PB} 4.2 / 6.2$ & $10.0 \mathrm{~PB} 4.2 / 6.6$ \\
\hline $10 \mathrm{~B} 4,8$ & .1292 & .2831 & $5 \mathrm{~PB} 4.1 / 7.0$ & $10.0 \mathrm{~B} 4.1 / 7.3$ \\
\hline P 4/10 & .1292 & .2828 & $5 \mathrm{~PB} 4.1 / 6.9$ & 5. $0 \mathrm{P} \quad 4.1 / 10.1$ \\
\hline $\mathrm{PB} \mathrm{4/8}$ & .1387 & .2783 & $5 \mathrm{~PB} 4.3 / 7.3$ & 4.5PB $4.3 / 7.4$ \\
\hline $10 \mathrm{~PB} 4 / 8$ & .1291 & .2685 & $5 \mathrm{~PB} 4.1 / 7.6$ & 10.0PB $4.1 / 8.5$ \\
\hline P $4 / 12$ & .1252 & .2504 & $5 \mathrm{~PB} 4.1 / 8.6$ & 5. $5 \mathrm{P} 4.1 / 12.6$ \\
\hline $10 \mathrm{~PB} 4 / 10$ & .1224 & .2192 & $5 \mathrm{~PB} 4.0 / 8.6$ & $10.0 \mathrm{~PB} 4.0 / 9.7$ \\
\hline PB 4/10 & .1446 & .2560 & $5 \mathrm{~PB} 4.4 / 8.9$ & $4.0 \mathrm{~PB} 4.4 / 8.9$ \\
\hline
\end{tabular}


TABLE 4. Deuteranopic arrangement-Continued

\begin{tabular}{|c|c|c|c|c|}
\hline \multirow{2}{*}{$\begin{array}{l}\text { Munsell } \\
\text { book nota- } \\
\text { tion }\end{array}$} & \multirow{2}{*}{$\begin{array}{c}\text { Deuteran- } \\
\text { opic re- } \\
\text { flectance, } \\
W_{d}=Y\end{array}$} & \multirow{2}{*}{$\begin{array}{c}\text { Deuteran- } \\
\text { opic chro- } \\
\text { maticity } \\
\text { coordinate, } \\
w_{d}\end{array}$} & \multicolumn{2}{|c|}{ Munsell renotations } \\
\hline & & & Deuteranopic & Normal \\
\hline $5 \mathrm{YR} 3 / 4$ & 0.0783 & 0.6552 & $5 \mathrm{Y} \quad 3.3 / 3.0$ & 5. 0 YR $3.3 / 4.1$ \\
\hline $10 \mathrm{R} 3 / 6$ & .0786 & .6380 & $5 \mathrm{Y} 3.3 / 2.8$ & $10.0 \mathrm{R} 3.3 / 5.4$ \\
\hline R $3 / 10$ & .0744 & .6332 & $5 Y \quad 3.2 / 2.7$ & 5. $0 \mathrm{R} 3.2 / 8.3$ \\
\hline $10 \mathrm{YR} 3 / 2$ & .0696 & 6231 & $5 Y \quad 3.1 / 2.5$ & 10.0YR $3.1 ; 2.7$ \\
\hline $10 \mathrm{GY} 3 / 4$ & .0756 & .6102 & $5 Y \quad 3.2 / 2.3$ & 10.0GY $3.2 / 4.5$ \\
\hline R $3 / 8$ & .0743 & .6055 & $5 \mathrm{Y} 3.2 / 2.2$ & $5.0 \mathrm{R} 3.2 / 7.4$ \\
\hline $10 \mathrm{R} 3 / 4$ & .0741 & .5961 & $5 \mathrm{Y} 3.2 / 2.1$ & $10.0 \mathrm{R} 3.2 / 4.1$ \\
\hline GY $3 / 4$ & .0739 & .5960 & $5 \mathrm{Y} 3.2 / 2.1$ & 5. $0 \mathrm{GY} 3.2 / 2.6$ \\
\hline $10 \mathrm{Y} 3 / 2$ & .0788 & .5685 & $5 Y \quad 3.3 / 1.7$ & $0.5 \mathrm{GY} 3.3 / 1.8$ \\
\hline R $3 / 6$ & .0829 & .5647 & $5 \mathrm{Y} \quad 3.4 / 1.7$ & 4. $5 \mathrm{R} 3.4 / 6.2$ \\
\hline YR $3 / 2$ & .0698 & .5602 & $5 \mathrm{Y} 3.1 / 1.6$ & 5. 0 YR $3.1 / 2.0$ \\
\hline YR 3/ 2 & .0701 & .5577 & $5 Y 3.1 / 1.5$ & 5. 0 YR $3.1 / 2.1$ \\
\hline GY $3 / 2$ & .0748 & .5533 & $5 \mathrm{Y} 3.2 / 1.4$ & 4. $5 \mathrm{GY} 3.2 / 1.9$ \\
\hline R $3 / 4$ & .0734 & .5506 & $5 \mathrm{Y} 3.2 / 1.4$ & 5.0R $3.2 / 4.6$ \\
\hline G $3 / 4$ & .0803 & .5407 & $5 Y \quad 3.3 / 1.3$ & 4. $0 \mathrm{G} 3.3 / 4.8$ \\
\hline Y $3 / 2$ & .0689 & .5362 & $5 \mathrm{Y} 3.1 / 1.2$ & $6.0 \mathrm{Y} 3.1 / 1.1$ \\
\hline G $3 / 2$ & .0703 & .4989 & $5 Y 3.1 / 0.6$ & $5.0 \mathrm{G} 3.1 / 2.7$ \\
\hline R $3 / 2$ & .0716 & .4979 & $5 \mathrm{Y} 3.1 / 0.6$ & $3.5 \mathrm{R} 3.1 / 2.1$ \\
\hline $10 \mathrm{RP} 3 / 8$ & .0698 & .4847 & $5 Y \quad 3.1 / 0.5$ & $9.0 \mathrm{RP} 3.1 / 7.0$ \\
\hline $10 \mathrm{RP} 3 / 6$ & .0712 & .4821 & $5 Y \quad 3.1 / 0.4$ & $9.0 \mathrm{RP} 3.1 / 5.6$ \\
\hline $10 \mathrm{G} 3 / 4$ & .0754 & .4736 & $5 \mathrm{Y} \quad 3.2 / 0.3$ & $10.0 \mathrm{G} 3.2 / 4.7$ \\
\hline 10RP 3/ 4 & .0658 & .4703 & $5 Y 3.0 / 0.3$ & 9.0RP $3.0 / 3.4$ \\
\hline $10 \mathrm{RP} 3 / 10$ & .0735 & .4640 & $5 \mathrm{Y} 3.2 / 0.1$ & 8. $5 \mathrm{RP} 3.2 / 8.5$ \\
\hline N 3/ & .0651 & .4511 & $5 \mathrm{~PB} 3.0 / 0.1$ & 2. $5 \mathrm{P} 3.0 / 0.2$ \\
\hline $\mathrm{RP} 3 / 2$ & .0654 & .4404 & $5 \mathrm{~PB} 3.0 / 0.3$ & 4.5RP $3.0 / 1.9$ \\
\hline BG $3 / 2$ & .0727 & .4327 & $5 \mathrm{~PB} 3.2 / 0.6$ & 5. $0 \mathrm{BG} 3.2 / 2.8$ \\
\hline RP 3/4 & .0675 & .4299 & $5 \mathrm{~PB} 3.0 / 0.6$ & 5. $0 \mathrm{RP} 3.0 / 3.7$ \\
\hline RP 3/ 6 & .0664 & .4229 & $5 \mathrm{~PB} 3.0 / 0.8$ & 5. $0 \mathrm{RP} 3.0 / 5.2$ \\
\hline BG $3 / 4$ & .0716 & .4180 & 5 PB $3.1 / 0.9$ & 5.0BG $3.1 / 4.1$ \\
\hline RP 3/ 8 & .0706 & .4060 & $5 \mathrm{~PB} 3.1 / 1.2$ & 5. 0RP $3.1 / 6.8$ \\
\hline RP $3 / 10$ & .0743 & .3975 & $5 \mathrm{~PB} 3.2 / 1.5$ & 5.0RP $3.2 / 8.4$ \\
\hline BG $3 / 6$ & .0767 & .3970 & $5 \mathrm{~PB} 3.2 / 1.5$ & 6. $0 \mathrm{BG} 3.2 / 5.3$ \\
\hline P $3 / 2$ & .0736 & .3870 & $5 \mathrm{~PB} 3.2 / 1.8$ & 5. $0 \mathrm{P} \quad 3.2 / 2.2$ \\
\hline B $3 / 2$ & .0715 & .3854 & 5PB 3.1/ 1.8 & 5. 5B 3.1/ 2.3 \\
\hline $10 \mathrm{BG} 3 / 4$ & .0778 & .3788 & $5 \mathrm{~PB} 3.3 / 2.0$ & $9.5 \mathrm{BG} 3.3 / 4.3$ \\
\hline PB $3 / 2$ & .0689 & .3710 & $5 \mathrm{~PB} 3.1 / 2.2$ & 5. $0 \mathrm{~PB} 3.1 / 2.1$ \\
\hline $10 \mathrm{P} 3 / 4$ & .0731 & .3699 & $5 \mathrm{~PB} 3.2 / 2.3$ & 10.0P $3.2 / 4.5$ \\
\hline $10 \mathrm{BG} 3 / 6$ & .0727 & .3475 & $5 \mathrm{~PB} \quad 3.2 / 2.9$ & $9.5 \mathrm{BG} 3.2 / 5.8$ \\
\hline B $3 / 4$ & .0700 & .3393 & $5 \mathrm{~PB} 3.1 / 3.1$ & 4. $5 \mathrm{~B} 3.1 / 4.2$ \\
\hline $10 \mathrm{P} 3 / 6$ & .0722 & .3327 & $5 \mathrm{~PB} 3.2 / 3.4$ & $9.0 \mathrm{P} 3.2 / 6.3$ \\
\hline P $3 / 4$ & .0705 & .3278 & $5 \mathrm{~PB} 3.1 / 3.5$ & $5.0 \mathrm{P} 3.1 / 4.5$ \\
\hline $10 \mathrm{~B} 3 / 4$ & .0708 & .3236 & 5PB 3.1/3.6 & 9. $5 \mathrm{~B} \quad 3.1 / 3.9$ \\
\hline $5 \mathrm{~PB} 3 / 4$ & .0699 & .3179 & 5PB $3.1 / 3.8$ & 5. $0 \mathrm{~PB} \quad 3.1 / 3.9$ \\
\hline $10 \mathrm{P} 3 / 8$ & .0700 & .3097 & $5 \mathrm{~PB} 3.1 / 4.1$ & 10.0P $3.1 / 8.3$ \\
\hline B $3 / 6$ & .0802 & .3047 & $5 \mathrm{~PB} 3.3 / 4.5$ & 5. 0 B $3.3 / 5.9$ \\
\hline $10 \mathrm{~PB} 3 / 4$ & .0713 & .3026 & $5 \mathrm{~PB} 3.1 / 4.5$ & 10.0PB $3.1 / 4.7$ \\
\hline P 3/ 6 & .0744 & .2950 & 5PB $3.2 / 4.8$ & 4. $5 \mathrm{P} \quad 3.2 / 6.4$ \\
\hline $10 \mathrm{P} 3 / 10$ & .0648 & .2767 & $5 \mathrm{~PB} 3.0 / 5.2$ & $9.0 \mathrm{P} 3.0 / 9.8$ \\
\hline $10 \mathrm{~B} 3 / 6$ & .0765 & .2840 & $5 \mathrm{~PB} 3.2 / 5.3$ & 9. $5 \mathrm{~B} \quad 3.2 / 5.6$ \\
\hline PB $3 / 6$ & .0749 & .2803 & $5 \mathrm{~PB} 3.2 / 5.5$ & 5. 0 PB $3.2 / 5.6$ \\
\hline $10 \mathrm{~PB} 3 / 6$ & .0711 & .2750 & $5 \mathrm{~PB} 3.1 / 5.5$ & 10.0PB $3.1 / 6.1$ \\
\hline P $3 / 8$ & .0759 & .2681 & $5 \mathrm{~PB} 3.2 / 6.0$ & 5. $0 \mathrm{P} \quad 3.2 / 8.4$ \\
\hline $10 \mathrm{~B} 3 / 8$ & .0812 & .2542 & 5PB $3.3 / 6.9$ & $9.0 \mathrm{~B} \quad 3.3 / 7.1$ \\
\hline $10 \mathrm{~PB} 3 / 8$ & .0724 & .2525 & $5 \mathrm{~PB} 3.2 / 6.8$ & 10.0PB $3.2 / 7.4$ \\
\hline PB 3/ 8 & .0768 & .2498 & $5 \mathrm{~PB} 3.2 / 7.0$ & 4. $5 \mathrm{~PB} 3.2 / 7.0$ \\
\hline P $3 / 10$ & .0769 & .2342 & 5PB 3. $2 / 7.8$ & 5. $0 \mathrm{P} 3.3 / 11.2$ \\
\hline
\end{tabular}

TABLE 4. Deuteranopic arrangement-Continued

\begin{tabular}{|c|c|c|c|c|}
\hline \multirow{2}{*}{$\begin{array}{l}\text { Munsell } \\
\text { book nota- } \\
\text { tion }\end{array}$} & \multirow{2}{*}{$\begin{array}{l}\text { Deuteran- } \\
\text { opic re- } \\
\text { flectance, } \\
W_{d}=Y\end{array}$} & \multirow{2}{*}{$\begin{array}{c}\text { Deuteran- } \\
\text { opic chro- } \\
\text { maticity } \\
\text { coordinate, } \\
w_{d}\end{array}$} & \multicolumn{2}{|c|}{ Munsell renotations } \\
\hline & & & Deuteranopic & Normal \\
\hline PB $3 / 10$ & 0.0844 & 0. 2323 & $5 \mathrm{~PB} 3.4 / 8.4$ & 4. $5 \mathrm{~PB} 3.4 / 8.5$ \\
\hline PB $3 / 12$ & .0802 & 2119 & $5 \mathrm{~PB} 3.3 / 9.4$ & 4. 0 PB $3.3 / 9.4$ \\
\hline $10 \mathrm{~PB} 3 / 10$ & .0693 & .1960 & $5 \mathrm{~PB} 3.1 / 9.7$ & 10. 0 P B $3.1 / 10.6$ \\
\hline $5 G Y 2 / 2$ & .0461 & .5595 & $5 \mathrm{Y} 2.5 / 1.6$ & $5.5 \mathrm{GY} \quad 2.5 / 2.2$ \\
\hline $10 \mathrm{Y} 2 / 2$ & .0484 & .5589 & $5 Y \quad 2.6 / 1.4$ & $10.0 \mathrm{Y} 2.6 / 1.6$ \\
\hline Y $2 / 2$ & .0472 & .5579 & $5 Y \quad 2.5 / 1.4$ & $5.0 \mathrm{Y} \quad 2.5 / .1 .4$ \\
\hline $10 \mathrm{YR} 2 / 2$ & .0505 & .5519 & $5 Y \quad 2.6 / 1.3$ & 7. 5 Y R $2.6 / 1.6$ \\
\hline YR $2 / 2$ & .0439 & .5420 & $5 \mathrm{Y} 2.4 / 1.2$ & 4. $5 \mathrm{YR} 2.4 / 1.8$ \\
\hline $10 \mathrm{GY} 2 / 2$ & .0440 & .5399 & $5 \mathrm{Y} \quad 2.4 / 1.1$ & $9.5 \mathrm{GY} 2.4 / 2.5$ \\
\hline $10 \mathrm{R} 2 / 2$ & .0458 & .5344 & $5 \mathrm{Y} \quad 2.5 / 1.0$ & 8. $5 \mathrm{R} 2.5 / 2.6$ \\
\hline R 2/4 & .0384 & .5168 & $5 \mathrm{Y} \quad 2.3 / 0.8$ & 4.0R $2.5 / 3.8$ \\
\hline $\mathrm{R} 2 / 6$ & .0461 & .5151 & $5 \mathrm{Y} \quad 2.5 / 0.8$ & $3.0 \mathrm{R} 2.5 / 4.8$ \\
\hline $\mathrm{R} \mathrm{2/2}$ & .0400 & .4878 & $5 Y \quad 2.3 / 0.5$ & 3. $5 \mathrm{R} \quad 2.3 / 2.2$ \\
\hline G 2/2 & .0373 & .4850 & $5 \mathrm{Y} \quad 2.2 / 0.4$ & $7.0 \mathrm{G} \quad 2.2 / 2.4$ \\
\hline $10 \mathrm{G} 2 / 2$ & .0407 & .4678 & $5 \mathrm{Y} \quad 2.3 / 0.2$ & $10.0 \mathrm{G} 2.3 / 3.1$ \\
\hline N 2/ & .0300 & .4505 & $5 \mathrm{~PB} 2.0 / 0.1$ & 5. 0 P $2.0 / 0.2$ \\
\hline 10RP $2 / 4$ & .0395 & .4458 & $5 \mathrm{~PB} \quad 2.3 / 0.1$ & 8.0RP $2.3 / 3.7$ \\
\hline RP 2/ 2 & .0386 & .4362 & $5 \mathrm{~PB} 2.3 / 0.3$ & 6. $0 \mathrm{RP} \quad 2.3 / 2.3$ \\
\hline BG 2/ 2 & .0379 & .4351 & $5 \mathrm{~PB} 2.2 / 0.3$ & 4. $5 \mathrm{BG} 2.2 / 2.5$ \\
\hline 10RP $2 / 6$ & .0416 & .4333 & $5 \mathrm{~PB} 2.4 / 0.4$ & 8.0RP 2.4/5.6 \\
\hline BG $2 / 4$ & .0395 & .4180 & $5 \mathrm{~PB} 2.3 / 0.7$ & $\begin{array}{llll}5.0 \mathrm{BG} & 2.3 / 3.7\end{array}$ \\
\hline $10 \mathrm{BG} 2 / 2$ & .0455 & .4070 & $5 \mathrm{~PB} 2.5 / 1.0$ & 7. $0 \mathrm{BG} 2.5 / 3.1$ \\
\hline RP 2/ 4 & .0411 & .4045 & $5 \mathrm{~PB} 2.4 / 1.0$ & 5. $0 \mathrm{RP} \quad 2.4 / 4.6$ \\
\hline B 2/ 2 & .0381 & .3952 & $5 \mathrm{~PB} 2.2 / 1.3$ & 2.0B $2.2 / 2.2$ \\
\hline RP 2/ 6 & .0440 & .3790 & $5 \mathrm{~PB} 2.4 / 1.7$ & 4. 0RP 2.4/5.9 \\
\hline P 2/ 2 & .0351 & .3506 & $5 \mathrm{~PB} 2.1 / 2.2$ & 5. $5 \mathrm{P} \quad 2.1 / 2.9$ \\
\hline PB 2/ 2 & .0353 & .3388 & $5 \mathrm{~PB} 2.2 / 2.5$ & 4. $5 \mathrm{~PB} 2.2 / 2.6$ \\
\hline $10 \mathrm{~B} 2 / 2$ & .0390 & .3353 & $5 \mathrm{~PB} 2.3 / 2.7$ & $8.5 \mathrm{~B} \quad 2.2 / 3.1$ \\
\hline $10 \mathrm{P} 2 / 4$ & .0370 & .3220 & $5 \mathrm{~PB} 2.2 / 3.0$ & 9. $0 \mathrm{P} \quad 2.2 / 5.3$ \\
\hline P 2/ 4 & .0358 & .3102 & $5 \mathrm{~PB} \quad 2.2 / 3.3$ & 5. $0 \mathrm{P} \quad 2.2 / 4.6$ \\
\hline $10 \mathrm{P} 2 / 6$ & .0377 & .2957 & $5 \mathrm{~PB} 2.2 / 3.7$ & 9. 0 P $2.2 / 6.8$ \\
\hline $10 \mathrm{~PB} 2 / 4$ & .0380 & .2770 & $5 \mathrm{~PB} 2.2 / 4.6$ & 10.0PB $2.2 / 4.8$ \\
\hline PB 2/ 4 & .0385 & .2715 & $5 \mathrm{~PB} 2.3 / 4.7$ & 4. $0 \mathrm{~PB} \quad 2.3 / 4.8$ \\
\hline $10 \mathrm{~PB} 2 / 6$ & $\because 0358$ & .2660 & $5 \mathrm{~PB} 2.2 / 4.6$ & 10.0PB $2.2 / 5.5$ \\
\hline P $2 / 6$ & .0346 & .2659 & $5 \mathrm{~PB} 2.1 / 4.6$ & 5.0P $2.1 / 6.8$ \\
\hline $\mathrm{PB} \mathrm{2/6}$ & .0405 & .2446 & 5 PB $2.3 / 5.8$ & 4. $0 \mathrm{~PB} \quad 2.3 / 5.9$ \\
\hline
\end{tabular}

TABLE 5. Protanopic arrangement

\begin{tabular}{|c|c|c|c|c|}
\hline \multirow{2}{*}{$\begin{array}{l}\text { Munsell } \\
\text { book } \\
\text { notation }\end{array}$} & \multirow{2}{*}{$\begin{array}{c}\text { Protanopic } \\
\text { reflectance, } \\
0.9733 W_{p}\end{array}$} & \multirow{2}{*}{$\begin{array}{l}\text { Protanopic } \\
\text { chroma- } \\
\text { ticity coor- } \\
\text { dinate, } w_{p}\end{array}$} & \multicolumn{2}{|c|}{ Munsell renotations } \\
\hline & & & Protanopic & Normal \\
\hline N 9 & 0.7244 & 0.4708 & $5 Y \quad 8.7 / 0.2$ & $7.5 \mathrm{Y} 8.7 / 0.1$ \\
\hline $10 \mathrm{BG} 8 / 2$ & .6464 & .4705 & $5 \mathrm{Y} 8.3 / 0.2$ & 5. $0 \mathrm{BG} 8.2 / 1.9$ \\
\hline $5 \mathrm{~B} 8 / 2$ & .6391 & .4511 & 5 PB $8.3 / 1.3$ & 4. $0 \mathrm{~B} 8.2 / 2.2$ \\
\hline $10 \mathrm{~B} 8 / 2$ & .6732 & .4317 & 5 PB $8.4 / 2.8$ & 1. $0 \mathrm{~PB} 8.3 / 3.0$ \\
\hline $5 \mathrm{~PB} 8 / 2$ & .6543 & .4267 & 5 PB $8.3 / 3.2$ & 5. 0 PB $8.3 / 3.2$ \\
\hline $10 \mathrm{~PB} 8 / 2$ & .6608 & .4205 & $5 \mathrm{~PB} 8.4 / 3.5$ & $\begin{array}{l}7.5 \mathrm{~PB} 8.3 / 3.4 \\
\end{array}$ \\
\hline $10 \mathrm{Y} 8 / 8$ & .5773 & .7889 & $5 Y 7.9 / 9.3$ & $0.5 \mathrm{GY} 8.1 / 9.1$ \\
\hline $5 G Y 8 / 8$ & .5991 & .7505 & $5 \mathrm{Y} 8.0 / 8.3$ & 4. $5 \mathrm{GY} 8.1 / 8.7$ \\
\hline $5 \mathrm{GY} 8 / 6$ & .5914 & .6659 & $5 Y 8.0 / 5.9$ & 5. $5 \mathrm{GY} 8.0 / 6.2$ \\
\hline $10 \mathrm{Y} 8 / 6$ & .5817 & .6657 & $5 Y 8.0 / 5.8$ & $0.5 \mathrm{GY} 8.0 / 5.7$ \\
\hline $5 Y 8 / 6$ & .5451 & .6609 & $5 Y 7.7 / 5.7$ & 4. $5 \mathrm{Y} \quad 7.9 / 5.8$ \\
\hline
\end{tabular}


TABLE 5. Protanopic arrangement-Continued

\begin{tabular}{|c|c|c|c|c|}
\hline \multirow{2}{*}{$\begin{array}{c}\text { Munsell } \\
\text { book } \\
\text { notation }\end{array}$} & \multirow{2}{*}{$\begin{array}{c}\text { Protanopic } \\
\text { reflectance, } \\
0.9733 W_{p}\end{array}$} & \multirow{2}{*}{$\begin{array}{l}\text { Protanopic } \\
\text { chroma- } \\
\text { ticity coor- } \\
\text { dinate, } w_{p}\end{array}$} & \multicolumn{2}{|c|}{ Munsell renotations } \\
\hline & & & Protanopic & Normal \\
\hline $10 \mathrm{YR} 8 / 6$ & 0.5632 & 0.6134 & $5 Y 7.8 / 4.3$ & 9.0YR $8.1 / 5.5$ \\
\hline $10 \mathrm{GY} 8 / 6$ & .5987 & .5866 & $5 \mathrm{Y} 8.0 / 3.6$ & 9.5GY $7.9 / 5.1$ \\
\hline $10 \mathrm{Y} 8 / 4$ & .5873 & .5842 & $5 Y 8.0 / 3.5$ & 1.0GY $8.0 / 3.4$ \\
\hline $5 G Y 8 / 4$ & .6044 & .5786 & $5 Y 8.1 / 3.4$ & 6. $0 \mathrm{GY} 8.1 / 3.6$ \\
\hline $10 \mathrm{GY} 8 / 4$ & 6362 & .5545 & $5 Y 8.2 / 2.7$ & $0.5 \mathrm{G} 8.2 / 4.0$ \\
\hline $10 \mathrm{YR} 8 / 4$ & .5656 & .5512 & $5 Y 7.9 / 2.5$ & 9.0 Y R $8.0 / 3.4$ \\
\hline $5 \mathrm{YR} 8 / 4$ & .5614 & .5225 & $5 Y 7.8 / 1.6$ & 4. 0 YR $8.0 / 3.5$ \\
\hline $5 \mathrm{G} 8 / 6$ & .5954 & .5187 & $5 \mathrm{Y} 8.0 / 1.5$ & $6.5 \mathrm{G} 7.8 / 4.7$ \\
\hline $5 \mathrm{GY} 8 / 2$ & .5851 & .5174 & $5 \mathrm{Y} 8.0 / 1.5$ & 6. $0 \mathrm{GY} 8.0 / 1.6$ \\
\hline $5 \mathrm{G} 8 / 4$ & .6141 & .5075 & $5 Y 8.1 / 1.3$ & $5.5 \mathrm{G} 8.0 / 3.3$ \\
\hline $10 \mathrm{R} 8 / 4$ & .5725 & .5059 & $5 Y 7.9 / 1.2$ & $10.0 \mathrm{R} 8.1 / 3.7$ \\
\hline $5 \mathrm{G} 8 / 2$ & .5854 & .4984 & 5 Y $8.0 / 0.9$ & $4.5 G 7.9 / 2.1$ \\
\hline $10 \mathrm{G} 8 / 2$ & .6133 & .4943 & $5 Y 8.1 / 0.8$ & 5. $0 \mathrm{G} 8.0 / 2.0$ \\
\hline $5 \mathrm{YR} 8 / 2$ & .5731 & .4937 & $5 Y 7.9 / 0.8$ & 4. 0 Y R $8.0 / 2.0$ \\
\hline $5 \mathrm{BG} 8 / 2$ & 6281 & 4803 & $5 \mathrm{Y} 8.2 / 0.5$ & 1. $0 \mathrm{BG} 8.1 / 2.0$ \\
\hline $5 \mathrm{R} 8 / 4$ & .5565 & .4772 & $5 \mathrm{Y} 7.8 / 0.4$ & $3.5 \mathrm{R} 8.0 / 3.6$ \\
\hline N 8/ & .5741 & .4764 & $5 Y 7.9 / 0.3$ & 2. $5 \mathrm{GY} 7.9 / 0.3$ \\
\hline $5 \mathrm{R} 8 / 2$ & .5857 & .4698 & $5 Y 8.0 / 0.2$ & 2. $0 \mathrm{R} 8.1 / 2.2$ \\
\hline $5 \mathrm{RP} 8 / 2$ & .6317 & .4654 & N 8.2/ & 8. 0 RP $8.3 / 1.9$ \\
\hline $10 \mathrm{RP} 8 / 4$ & .5905 & .4662 & N $8.0 /$ & 9. $5 \mathrm{RP} 8.1 / 2.7$ \\
\hline $10 \mathrm{RP} 8 / 6$ & .5958 & .4641 & $5 \mathrm{~PB} 8.0 / 0.1$ & 8. $5 \mathrm{RP} 8.2 / 3.6$ \\
\hline $5 \mathrm{RP} 8 / 6$ & .5839 & .4586 & $5 \mathrm{~PB} 8.0 / 0.6$ & 6. $5 \mathrm{RP} 8.1 / 3.4$ \\
\hline $5 \mathrm{RP} 8 / 4$ & .5874 & .4582 & $5 \mathrm{~PB} 8.0 / 0.7$ & 5. $5 \mathrm{RP} 8.1 / 2.8$ \\
\hline 5 B $8 / 4$ & .6310 & .4452 & $5 \mathrm{~PB} 8.2 / 1.8$ & 4.0B $8.1 / 2.9$ \\
\hline $5 \mathrm{P} 8 / 2$ & .5877 & .4388 & $5 \mathrm{~PB} 8.0 / 2.2$ & $5.0 \mathrm{P} 8.0 / 2.5$ \\
\hline $10 \mathrm{P} 8 / 4$ & .6081 & .4316 & 5 PB $8.1 / 2.8$ & 7. $5 \mathrm{P} 8.2 / 3.5$ \\
\hline $5 \mathrm{P} 8 / 4$ & .5903 & .4054 & $5 \mathrm{~PB} 8.0 / 4.5$ & $4.5 \mathrm{P} 8.0 / 4.8$ \\
\hline $5 \mathrm{Y} 8 / 12$ & .5208 & .8848 & $5 \mathrm{Y} \quad 7.6 / 11.8$ & 5. $5 \mathrm{Y} \quad 7.9 / 12.3$ \\
\hline Y $8 / 10$ & .5292 & .8501 & $5 Y \quad 7.6 / 10.8$ & 5. $5 \mathrm{Y} \quad 7.9 / 11.1$ \\
\hline Y $8 / 8$ & .5393 & .7579 & $5 Y \quad 7.7 / 8.3$ & $5.5 \mathrm{Y} \quad 7.9 / 8.3$ \\
\hline $\mathrm{Y} 8 / 8$ & .5339 & .7525 & $5 Y \quad 7.7 / 8.2$ & $5.5 Y \quad 7.9 / 8.2$ \\
\hline $10 \mathrm{GY} 7 / 6$ & .4726 & .6222 & $5 \mathrm{Y} \quad 7.3 / 4.3$ & 10.0GY $7.1 / 6.5$ \\
\hline Y $8 / 4$ & .5426 & .5818 & $5 \mathrm{Y} \quad 7.7 / 3.4$ & $4.0 \mathrm{Y} 7.8 / 3.5$ \\
\hline $10 \mathrm{GY} 7 / 4$ & .4718 & .5631 & $5 Y \quad 7.3 / 2.7$ & $10.0 Y \quad 7.2 / 4.1$ \\
\hline G 7/ 6 & .4749 & .5299 & $5 \mathrm{Y} 7.3 / 1.8$ & $7.0 \mathrm{G} \quad 7.0 / 6.1$ \\
\hline Y $8 / 2$ & .5455 & .5156 & $5 Y \quad 7.7 / 1.5$ & $3.5 \mathrm{Y} 7.8 / 1.6$ \\
\hline $10 \mathrm{G} 7 / 4$ & .4960 & .4951 & $5 \mathrm{Y} \quad 7.4 / 0.8$ & $0.5 \mathrm{BG} 7.2 / 3.9$ \\
\hline $\mathrm{BG} 7 / 2$ & .4913 & .4752 & $5 Y \quad 7.4 / 0.3$ & $3.5 \mathrm{BG} 7.3 / 2.1$ \\
\hline $\mathrm{BG} 7 / 4$ & .4908 & .4733 & $5 Y \quad 7.4 / 0.2$ & 4. $5 \mathrm{BG} \quad 7.2 / 3.4$ \\
\hline $\mathrm{RP} 7 / 4$ & .4693 & .4558 & $5 \mathrm{~PB} \quad 7.3 / 0.8$ & 6.0RP $7.4 / 3.3$ \\
\hline B $7 / 2$ & .4895 & .4505 & $5 \mathrm{~PB} \quad 7.4 / 1.2$ & $3.5 \mathrm{~B} \quad 7.3 / 2.2$ \\
\hline $10 \mathrm{BG} 7 / 4$ & .4863 & .4471 & $5 \mathrm{~PB} 7.4 / 1.4$ & $0.5 \mathrm{~B} \quad 7.2 / 3.6$ \\
\hline $\mathrm{RP} 7 / 6$ & .4687 & .4463 & $5 \mathrm{~PB} 7.3 / 1.6$ & 5. $0 \mathrm{RP} 7.4 / 4.4$ \\
\hline B $7 / 4$ & .4875 & .4313 & $5 \mathrm{~PB} 7.4 / 2.5$ & 5.0B $7.2 / 3.7$ \\
\hline $\mathrm{PB} 7 / 2$ & .5049 & .4260 & $5 \mathrm{~PB} 7.5 / 2.9$ & 3. $5 \mathrm{~PB} 7.4 / 3.0$ \\
\hline B $7 / 6$ & .4798 & .4213 & $5 \mathrm{~PB} \quad 7.3 / 3.1$ & 5. $0 \mathrm{~B} \quad 7.1 / 4.7$ \\
\hline 10B $7 / 4$ & .4794 & .4158 & $5 \mathrm{~PB} 7.3 / 3.5$ & $9.0 \mathrm{~B} \quad 7.2 / 4.0$ \\
\hline $\mathrm{PB} 7 / 4$ & .4942 & .4048 & $5 \mathrm{~PB} 7.4 / 4.3$ & 4.0 \\
\hline $10 \mathrm{~B} 7 / 6$ & .4959 & .3963 & $5 \mathrm{~PB} \quad 7.5 / 4.9$ & $\begin{array}{lll}9.5 \mathrm{~B} & 7.2 / 5.7\end{array}$ \\
\hline PB 7/ 6 & .4817 & .3878 & $5 \mathrm{~PB} \quad 7.3 / 5.4$ & 4. $0 \mathrm{~PB} \quad 7.2 / 5.6$ \\
\hline $10 \mathrm{Y} 7 / 8$ & .4214 & .8324 & $5 \mathrm{Y} 6.9 / 9.5$ & $10.0 \mathrm{Y} 7.1 / 9.3$ \\
\hline GY $7 / 10$ & .4235 & .8191 & $5 Y \quad 7.0 / 9.2$ & 4. $0 \mathrm{GY} \quad 7.0 / 9.5$ \\
\hline GY $7 / 8$ & .4506 & .7486 & $5 Y 7.1 / 7.5$ & 5. $0 \mathrm{GY} 7.1 / 8.0$ \\
\hline $10 \mathrm{Y} 7 / 6$ & .4176 & .7387 & $5 \mathrm{Y} \quad 6.9 / 7.0$ & $0.5 \mathrm{GY} \quad 7.0 / 6.9$ \\
\hline GY $7 / 6$ & .4502 & .6922 & $5 Y \quad 7.1 / 6.1$ & 5.0GY $7.1 / 6.4$ \\
\hline $10 \mathrm{GY} 7 / 8$ & .4655 & .6697 & $5 \mathrm{Y} 7.2 / 5.5$ & $10.0 \mathrm{GY} 7.1 / 8.3$ \\
\hline $10 \mathrm{Y} 7 / 4$ & .4340 & .6064 & $5 \mathrm{Y} 7.0 / 3.8$ & 1.0GY $7.1 / 3.6$ \\
\hline
\end{tabular}

TABLE 5. Protanopic arrangement-Continued

\begin{tabular}{|c|c|c|c|c|}
\hline \multirow{2}{*}{$\begin{array}{l}\text { Munsell } \\
\text { book } \\
\text { notation }\end{array}$} & \multirow{2}{*}{$\begin{array}{c}\text { Protanopic } \\
\text { reflectance, } \\
0.9733 W_{p}\end{array}$} & \multirow{2}{*}{$\begin{array}{l}\text { Protanopic } \\
\text { chroma- } \\
\text { ticity coor- } \\
\text { dinate, } w_{p}\end{array}$} & \multicolumn{2}{|c|}{ Munsell renotations } \\
\hline & & & Protanopic & Normal \\
\hline GY 7/ 4 & 0.4364 & 0.5973 & $5 \mathrm{Y} 7.0 / 3.6$ & $5.5 \mathrm{GY} 7.0 / 3.8$ \\
\hline Y 7/ 4 & .4079 & .6033 & $5 Y 6.8 / 3.5$ & $4.0 Y \quad 7.0 / 3.8$ \\
\hline $10 Y R 7 / 4$ & .4296 & .5746 & $5 \mathrm{Y} \quad 7.0 / 2.9$ & 9.0 YR $7.2 / 3.8$ \\
\hline YR $7 / 4$ & .4016 & 5422 & $5 \mathrm{Y} 6.8 / 2.0$ & 3.0 YR $7.0 / 4.1$ \\
\hline $10 \mathrm{R} 7 / 6$ & .4036 & .5329 & $5 Y \quad 6.8 / 1.8$ & $9.0 \mathrm{R} \quad 7.2 / 5.7$ \\
\hline GY $7 / 2$ & .4261 & .5244 & $5 Y 7.0 / 1.6$ & 5.5GY $7.0 / 1.8$ \\
\hline $\mathrm{Y} 7 / 2$ & 4359 & .5213 & $5 \mathrm{Y} \quad 7.0 / 1.6$ & 4. $0 \mathrm{Y} \quad 7.1 / 1.6$ \\
\hline G 7/ 4 & .4613 & .5168 & $5 \mathrm{Y} \quad 7.2 / 1.4$ & $6.5 \mathrm{G} \quad 7.0 / 4.3$ \\
\hline $10 \mathrm{R} 7 / 4$ & .4122 & .5145 & $5 \mathrm{Y} 6.9 / 1.3$ & 8.5R $7.2 / 4.7$ \\
\hline $5 \mathrm{YR} 7 / 2$ & .4183 & .5013 & $5 \mathrm{Y} 6.9 / 1.0$ & $3.0 Y R \quad 7.0 / 2.1$ \\
\hline G $7 / 2$ & .4371 & .4926 & $5 Y 7.0 / 0.7$ & $6.5 \mathrm{G} \quad 7.0 / 2.1$ \\
\hline $\mathrm{R} 7 / 6$ & .3999 & .4899 & $5 \mathrm{Y} \quad 6.8 / 0.6$ & $3.5 \mathrm{R} 7.1 / 5.7$ \\
\hline R $7 / 4$ & .4012 & .4809 & $5 \mathrm{Y} 6.8 / 0.4$ & $3.0 \mathrm{R} 7.1 / 5.2$ \\
\hline R 7/ 2 & .4336 & .4729 & $5 \mathrm{Y} 7.0 / 0.2$ & $2.5 \mathrm{R} \quad 7.2 / 2.6$ \\
\hline 10RP 7/ 4 & .4154 & .4657 & N 6.9/ & 9.5RP $7.1 / 3.5$ \\
\hline $10 \mathrm{RP} 7 / 6$ & .4213 & .4649 & N $6.9 /$ & 9.5RP $7.2 / 4.7$ \\
\hline N 7/ & .4441 & .4633 & $5 \mathrm{~PB} \quad 7.1 / 0.1$ & 7. 5 PB $7.1 / 0.1$ \\
\hline $10 \mathrm{RP} 7 / 8$ & .3999 & .4625 & $5 \mathrm{~PB} 6.8 / 0.2$ & 9.0RP $7.1 / 5.8$ \\
\hline RP 7/ 2 & .4599 & .4600 & 5 PB $7.2 / 0.4$ & 6.5RP $7.3 / 2.3$ \\
\hline $\mathrm{RP} 7 / 8$ & .4540 & .4400 & $5 \mathrm{~PB} 7.2 / 1.9$ & 4.5RP $7.4 / 4.9$ \\
\hline $10 \mathrm{P} 7 / 4$ & .4510 & .4273 & $5 \mathrm{~PB} 7.1 / 2.7$ & $10.0 \mathrm{P} 7.2 / 4.0$ \\
\hline P $7 / 2$ & .4085 & .4259 & 5 PB $6.8 / 2.7$ & 4. 5 P $6.9 / 2.7$ \\
\hline $10 \mathrm{P} 7 / 6$ & .4505 & .4204 & $5 \mathrm{~PB} 7.1 / 3.1$ & $9.5 \mathrm{P} 7.2 / 4.6$ \\
\hline $10 \mathrm{~PB} 7 / 4$ & .4544 & .4072 & $5 \mathrm{~PB} 7.2 / 4.0$ & 10.0PB $7.1 / 4.0$ \\
\hline $10 \mathrm{P} 7 / 8$ & .4489 & .4061 & $5 \mathrm{~PB} 7.1 / 4.2$ & 9.5P $7.3 / 6.0$ \\
\hline P 7/ 4 & .4419 & .4005 & $5 \mathrm{~PB} 7.1 / 4.4$ & $5.0 \mathrm{P} \quad 7.1 / 4.8$ \\
\hline $10 \mathrm{~PB} 7 / 6$ & .4435 & .3838 & $5 \mathrm{~PB} 7.1 / 5.6$ & 10. 0 PB $7.0 / 5.3$ \\
\hline P 7/ 6 & .4283 & .3775 & $5 \mathrm{~PB} 7.0 / 5.8$ & 5. $5 \mathrm{P} \quad 7.0 / 6.4$ \\
\hline Y $7 / 10$ & .3814 & .8415 & $5 Y \quad 6.6 / 9.5$ & $\begin{array}{lll}5.0 Y & 6.9 / 9.7\end{array}$ \\
\hline Y $7 / 8$ & .3879 & .8353 & $5 Y \quad 6.7 / 9.4$ & $6.0 \mathrm{Y} 6.9 / 9.5$ \\
\hline $10 \mathrm{YR} 7 / 10$ & .3602 & .8235 & $5 Y \quad 6.5 / 8.7$ & 1. $0 \mathrm{Y} 6.9 / 10.5$ \\
\hline $10 \mathrm{YR} 7 / 8$ & .3885 & .7388 & $5 Y \quad 6.7 / 6.9$ & $0.5 \mathrm{Y} 7.1 / 8.3$ \\
\hline YR $7 / 10$ & .3443 & .7299 & $5 \mathrm{Y} \quad 6.4 / 6.4$ & 5. 5 YR $6.9 / 10.1$ \\
\hline Y 7/ 6 & .3854 & .7188 & $5 \mathrm{Y} 6.7 / 6.4$ & $\begin{array}{llll}4.5 Y & 6.9 / 6.6\end{array}$ \\
\hline $10 \mathrm{GY} 6 / 8$ & .3403 & .6868 & $5 \mathrm{Y} \quad 6.3 / 5.3$ & $9.0 \mathrm{GY} 6.2 / 7.7$ \\
\hline $10 \mathrm{YR} 7 / 6$ & .3919 & .6650 & $5 Y \quad 6.7 / 5.2$ & 10.0YR $7.0 / 6.3$ \\
\hline YR 7/ 8 & .3696 & .6548 & $5 Y \quad 6.6 / 4.7$ & $4.5 \mathrm{YR} 7.0 / 8.2$ \\
\hline $10 \mathrm{GY} 6 / 6$ & .3343 & .6486 & $5 \mathrm{Y} 6.3 / 4.5$ & 10.0GY 6.1/6.7 \\
\hline YR 7/ 6 & .3757 & .5906 & $5 Y \quad 6.6 / 3.2$ & $3.5 \mathrm{YR} 7.0 / 6.0$ \\
\hline YR 7/ 6 & .3682 & .5894 & $5 Y \quad 6.5 / 3.2$ & 3.5 YR $6.9 / 5.9$ \\
\hline $10 \mathrm{R} 7 / 8$ & .3635 & .5736 & $5 Y \quad 6.5 / 2.8$ & $10.0 \mathrm{R} 7.0 / 7.5$ \\
\hline G $6 / 6$ & .3378 & .5566 & $5 \mathrm{Y} \quad 6.3 / 2.3$ & $6.0 \mathrm{G} 6.0 / 7.4$ \\
\hline G 6/ 4 & .3377 & .5259 & $5 \mathrm{Y} 6.3 / 1.5$ & 6. $0 \mathrm{G} 6.1 / 4.6$ \\
\hline $10 \mathrm{G} 6 / 6$ & .3889 & .5060 & $5 \mathrm{Y} 6.7 / 1.1$ & 1. 0 BG $6.4 / 6.0$ \\
\hline $10 \mathrm{G} 6 / 4$ & .3743 & .4943 & $5 Y \quad 6.6 / 0.7$ & 2.0BG $6.4 / 4.5$ \\
\hline R 7/ 8 & .3698 & .4911 & $5 \mathrm{Y} \quad 6.6 / 0.7$ & $3.5 \mathrm{R} \quad 7.0 \prime 7.0$ \\
\hline BG $6 / 2$ & .3593 & .4708 & $5 Y \quad 6.5 / 0.2$ & 5. 0 BG $6.4 / 2.4$ \\
\hline BG $6 / 4$ & .3700 & .4690 & $5 \mathrm{Y} 6.6 / 0.1$ & 5. $5 \mathrm{BG} 6.4 / 4.1$ \\
\hline $5 \mathrm{BG} 6 / 6$ & .3810 & .4676 & $5 \mathrm{Y} \quad 6.6 / 0.1$ & 5. $5 \mathrm{BG} 6.4 / 5.2$ \\
\hline $10 \mathrm{BG} 6 / 4$ & .3593 & .4426 & $5 \mathrm{~PB} 6.5 / 1.5$ & 10.0BG $6.3 / 4.1$ \\
\hline $10 \mathrm{BG} 6 / 6$ & .3776 & .4334 & $5 \mathrm{~PB} 6.6 / 2.1$ & 10.0BG $6.3 / 5.8$ \\
\hline B $6 / 2$ & .3400 & .4296 & $5 \mathrm{~PB} \quad 6.3 / 2.2$ & 7. 5 B $6.2 / 3.0$ \\
\hline PB 6/ 2 & .3530 & .4141 & $5 \mathrm{~PB} 6.4 / 3.2$ & 5. $0 \mathrm{~PB} 6.4 / 3.2$ \\
\hline B $6 / 4$ & .3527 & .4119 & $5 \mathrm{~PB} 6.4 / 3.4$ & $6.5 \mathrm{~B} \quad 6.2 / 4.5$ \\
\hline $10 \mathrm{~B} 6 / 4$ & .3665 & .4010 & $5 \mathrm{BP} 6.5 / 4.0$ & $10.0 \mathrm{~B} \quad 6.3 / 4.6$ \\
\hline B $6 / 6$ & .3527 & .3931 & $5 \mathrm{~PB} 6.4 / 4.5$ & 5. $5 \mathrm{~B} \quad 6.1 / 6.4$ \\
\hline $\mathrm{PB} 6 / 4$ & .3759 & .3927 & $5 \mathrm{~PB} 6.6 / 4.7$ & 5. 0 PB $6.5 / 4.7$ \\
\hline $10 \mathrm{~B} 6 / 6$ & .3654 & .3843 & $5 \mathrm{~PB} 6.5 / 5.1$ & $9.0 \mathrm{~B} 6.3 / 6.3$ \\
\hline
\end{tabular}


TABle 5. Protanopic arrangement-Continued

\begin{tabular}{|c|c|c|c|c|}
\hline \multirow{2}{*}{$\begin{array}{l}\text { Munsell } \\
\text { book } \\
\text { notation }\end{array}$} & \multirow{2}{*}{$\begin{array}{l}\text { Protanopic } \\
\text { reflectance, } \\
0.9733 W_{p}\end{array}$} & \multirow{2}{*}{$\begin{array}{l}\text { Protanopic } \\
\text { chroma- } \\
\text { ticity coor- } \\
\text { dinate, } w_{p}\end{array}$} & \multicolumn{2}{|c|}{ Munsell renotations } \\
\hline & & & Protanopic & Normal \\
\hline $10 \mathrm{~PB} 6 / 4$ & 0.3436 & 0.3821 & $5 \mathrm{~PB} 6.4 / 5.2$ & $0.5 \mathrm{P} \quad 6.3 / 4.8$ \\
\hline $\mathrm{PB} 6 / 6$ & .3634 & .3659 & $5 \mathrm{~PB} 6.5 / 6.3$ & 5. $0 \mathrm{~PB} \quad 6.3 / 6.2$ \\
\hline PB 6/ 8 & .3575 & .3624 & $5 \mathrm{~PB} 6.5 / 6.4$ & 5. $0 \mathrm{~PB} \quad 6.3 / 7.4$ \\
\hline $10 \mathrm{YR} 6 / 10$ & .3090 & .8170 & $5 \mathrm{Y} 6.1 / 8.2$ & 10.0YR $6.5 / 9.8$ \\
\hline $\mathrm{Y} 6 / 8$ & .2967 & .8224 & $5 Y 6.0 / 8.1$ & $5.5 Y \quad 6.2 / 8.3$ \\
\hline Y 6/ 8 & .2876 & .8199 & $5 \mathrm{Y} 5.9 / 8.0$ & 5. $5 \mathrm{Y} \quad 6.1 / 8.1$ \\
\hline $10 \mathrm{Y} 6 / 6$ & .3115 & .7940 & $5 Y \quad 6.1 / 7.7$ & $9.5 \mathrm{Y} \quad 6.2 / 7.4$ \\
\hline $\mathrm{Y}$ 6/ 6 & .2924 & .7718 & $5 \mathrm{Y} 5.9 / 7.0$ & 5.0Y $6.2 / 7.2$ \\
\hline YR $6 / 10$ & .2735 & .7544 & $5 Y 5.8 / 6.5$ & $6.0 \mathrm{YR} 6.3 / 9.8$ \\
\hline GY 6/ 8 & .3222 & .7499 & $5 \mathrm{Y} 6.2 / 6.7$ & 4.0GY $6.2 / 7.2$ \\
\hline $10 \mathrm{YR} 6 / 8$ & .3013 & .7449 & $5 \mathrm{Y} 6.0 / 6.5$ & $9.5 \mathrm{YR} 6.4 / 8.0$ \\
\hline GY 6/ 6 & .3198 & .7299 & $5 \mathrm{Y} 6.2 / 6.2$ & 5.0GY $6.2 / 6.7$ \\
\hline $10 \mathrm{GY} 6 / 10$ & .3224 & .7206 & $5 \mathrm{Y} 6.2 / 6.1$ & $9.0 \mathrm{GY} 6.0 / 8.7$ \\
\hline $10 \mathrm{YR} 6 / 6$ & .3003 & .6953 & $5 \mathrm{Y} 6.0 / 5.4$ & $9.5 \mathrm{YR} 6.3 / 6.7$ \\
\hline YR 6/ 8 & .2731 & .6871 & $5 Y \quad 5.8 / 5.0$ & $5.0 \mathrm{YR} 6.2 / 8.3$ \\
\hline $10 \mathrm{Y} 6 / 4$ & .3188 & .6400 & $5 Y \quad 6.2 / 4.2$ & $10.0 \mathrm{Y} \quad 6.2 / 4.1$ \\
\hline Y 6/ 4 & .2930 & .6317 & $5 \mathrm{Y} 5.9 / 3.9$ & 4. $5 \mathrm{Y} \quad 6.1 / 4.0$ \\
\hline GY 6/ 4 & .3212 & .6211 & $5 \mathrm{Y} \quad 6.2 / 3.8$ & 5.0GY $6.2 / 4.0$ \\
\hline YR 6/ 6 & .2808 & .6153 & $5 \mathrm{Y} 5.8 / 3.5$ & 4.5YR 6.2/6.0 \\
\hline $10 \mathrm{R} 6 / 8$ & .2737 & .6060 & $5 Y \quad 5.8 / 3.3$ & $0.5 \mathrm{YR} 6.3 / 8.2$ \\
\hline $10 \mathrm{YR} 6 / 4$ & .2916 & .6055 & $5 Y \quad 5.9 / 3.3$ & $0.5 \mathrm{Y} \quad 6.1 / 4.1$ \\
\hline $10 \mathrm{GY} 6 / 4$ & .3239 & .5772 & $5 \mathrm{Y} 6.2 / 2.8$ & $0.5 \mathrm{G} \quad 6.1 / 4.4$ \\
\hline $10 \mathrm{R} 6 / 6$ & .2898 & .5683 & $5 Y 5.9 / 2.4$ & $0.5 \mathrm{YR} 6.3 / 6.5$ \\
\hline YR 6/ 4 & .2826 & .5580 & $5 Y 5.8 / 2.2$ & 4.0YR $6.1 / 4.0$ \\
\hline Y 6/ 2 & .3127 & .5319 & $5 Y \quad 6.1 / 1.7$ & $\begin{array}{llll}4.0 \mathrm{Y} & 6.2 / 1.7\end{array}$ \\
\hline GY 6/ 2 & .3243 & .5305 & $5 Y \quad 6.2 / 1.7$ & 5. $0 \mathrm{GY} \quad 6.2 / 1.7$ \\
\hline $10 \mathrm{R} 6 / 4$ & .2944 & .5244 & $5 Y \quad 5.9 / 1.4$ & $9.0 \mathrm{R} 6.2 / 4.2$ \\
\hline 5 YR $6 / 2$ & .2977 & .5103 & $5 \mathrm{Y} 6.0 / 1.1$ & 4. $5 \mathrm{YR} 6.1 / 2.0$ \\
\hline $5 \mathrm{G} 6 / 2$ & .3158 & .5006 & $5 \mathrm{Y} 6.1 / 0.9$ & $6.0 \mathrm{G} 6.0 / 2.4$ \\
\hline $\mathrm{R}$ 6/ 6 & .2879 & .4921 & $5 Y 5.9 / 0.6$ & $3.5 \mathrm{R} \quad 6.2 / 6.3$ \\
\hline $\mathrm{R}$ 6/ 4 & .3004 & .4888 & $5 Y \quad 6.0 / 0.6$ & 4.5R $6.3 / 4.5$ \\
\hline $\mathrm{R} 6 / 2$ & .3060 & .4758 & $5 \mathrm{Y} 6.0 / 0.2$ & 4. $0 \mathrm{R} \quad 6.2 / 2.3$ \\
\hline N 6/ & .3032 & .4653 & N $6.0 /$ & N 6.0/ \\
\hline $10 \mathrm{RP} 6 / 6$ & .2915 & .4596 & 5 PB 5.9/ 0.4 & 9.0RP 6.2/ 5.5 \\
\hline $10 \mathrm{RP} 6 / 4$ & .3019 & .4593 & $5 \mathrm{~PB} 6.0 / 0.3$ & 8.5RP $6.2 / 4.0$ \\
\hline $10 \mathrm{RP} 6 / 8$ & .2811 & .4583 & 5 PB $5.8 / 0.4$ & $9.0 \mathrm{RP} 6.2 / 7.0$ \\
\hline RP 6/ 2 & .3072 & .4538 & $5 \mathrm{~PB} 6.1 / 0.8$ & 4.0RP $6.1 / 2.2$ \\
\hline $\mathrm{RP} 6 / 4$ & .3260 & .4428 & $5 \mathrm{~PB} 6.2 / 1.4$ & 4. $5 \mathrm{RP} 6.4 / 4.2$ \\
\hline $\mathrm{RP} 6 / 6$ & .3057 & .4347 & $5 \mathrm{~PB} 6.0 / 1.8$ & 4.5RP $6.3 / 5.6$ \\
\hline RP 6/ 8 & .2968 & .4208 & $5 \mathrm{~PB} 6.0 / 2.6$ & 5.0RP $6.3 / 7.3$ \\
\hline P 6/ 2 & .3218 & .4176 & $5 \mathrm{~PB} 6.2 / 2.9$ & 4. $5 \mathrm{P} \quad 6.2 / 3.0$ \\
\hline $10 \mathrm{P} 6 / 4$ & .3098 & .4135 & $5 \mathrm{~PB} 6.1 / 3.1$ & 10.0P $6.2 / 4.6$ \\
\hline $\mathrm{RP} 6 / 10$ & .2951 & .4086 & $5 \mathrm{~PB} 6.0 / 3.3$ & 4.5RP $6.3 / 8.5$ \\
\hline $10 \mathrm{P} 6 / 6$ & .3121 & .3931 & $5 \mathrm{~PB} 6.1 / 4.4$ & $9.5 \mathrm{P} \quad 6.2 / 6.2$ \\
\hline P 6/ 4 & .3270 & .3882 & $5 \mathrm{~PB} 6.2 / 4.7$ & 4.0P $6.2 / 4.9$ \\
\hline $10 \mathrm{P} 6 / 8$ & .3013 & .3710 & $5 \mathrm{~PB} 6.0 / 5.6$ & 9.0P $6.2 / 8.0$ \\
\hline P $6 / 6$ & .3126 & .3569 & $5 \mathrm{~PB} 6.1 / 6.7$ & 4.5P $6.2 / 7.1$ \\
\hline $10 \mathrm{~PB} 6 / 6$ & .3227 & .3564 & $5 \mathrm{~PB} 6.2 / 6.7$ & $10.0 \mathrm{~PB} 6.1 / 6.3$ \\
\hline P $6 / 8$ & .3080 & .3322 & $5 \mathrm{~PB} 6.1 / 8.4$ & 5.0P $6.1 / 9.1$ \\
\hline $10 \mathrm{~PB} 6 / 8$ & .3277 & .3322 & $5 \mathrm{~PB} 6.2 / 8.4$ & $0.5 \mathrm{P} \quad 6.2 / 8.0$ \\
\hline YR 6/12 & .2572 & .8107 & $5 \mathrm{Y} 5.6 / 7.5$ & $6.0 \mathrm{YR} 6.2 / 11.5$ \\
\hline $10 \mathrm{GY} 5 / 6$ & .2272 & .6835 & $5 \mathrm{Y} 5.3 / 4.6$ & 9.5GY $5.2 / 7.1$ \\
\hline $10 \mathrm{R} 6 / 10$ & .2509 & .6572 & $5 \mathrm{Y} 5.6 / 4.2$ & $0.5 \mathrm{YR} \quad 6.2 / 10.1$ \\
\hline $10 \mathrm{G} 5 / 6$ & .2292 & .5159 & $5 Y \quad 5.3 / 1.2$ & 1.0BG $5.0 / 7.0$ \\
\hline R 6/10 & .2571 & .5124 & $5 \mathrm{Y} \quad 5.6 / 1.1$ & $4.0 \mathrm{R} 6.2 / 9.7$ \\
\hline $\mathrm{R} 6 / 8$ & .2692 & .5045 & $5 \mathrm{Y} 5.7 / 0.9$ & 4. $0 \mathrm{R} 6.2 / 8.1$ \\
\hline
\end{tabular}

TABle 5. Protanopic arrangement-Continued

\begin{tabular}{|c|c|c|c|c|}
\hline \multirow{2}{*}{$\begin{array}{l}\text { Munsell } \\
\text { book } \\
\text { notation }\end{array}$} & \multirow{2}{*}{$\begin{array}{c}\text { Protanopic } \\
\text { reflectance, } \\
0.9733 W_{p}\end{array}$} & \multirow{2}{*}{$\begin{array}{l}\text { Protanopic } \\
\text { chroma- } \\
\text { ticity coor- } \\
\text { dinate, } w_{p}\end{array}$} & \multicolumn{2}{|c|}{ Munsell renotations } \\
\hline & & & Protanopic & Normal \\
\hline $10 \mathrm{G} 5 / 4$ & 0.2235 & 0.4993 & $5 \mathrm{Y} 5.3 / 0.8$ & $0.5 \mathrm{BG} 5.1 / 4.6$ \\
\hline $\mathrm{BG} 5 / 6$ & .2393 & .4643 & $5 \mathrm{~PB} \mathrm{5.4/0.1}$ & 5.0BG $5.1 / 6.4$ \\
\hline BG $5 / 4$ & .2320 & .4623 & $5 \mathrm{~PB} 5.4 / 0.2$ & 5. $5 \mathrm{BG} 5.2 / 4.4$ \\
\hline $10 \mathrm{RP} 6 / 10$ & .2660 & .4563 & $5 \mathrm{~PB} 5.7 / 0.6$ & 9.5RP $6.2 / 8.8$ \\
\hline $10 \mathrm{BG} 5 / 4$ & .2276 & .4303 & $5 \mathrm{~PB} 5.3 / 1.8$ & $0.5 \mathrm{~B} \quad 5.1 / 4.4$ \\
\hline $10 \mathrm{BG} 5 / 6$ & .2493 & .4091 & $5 \mathrm{~PB} 5.5 / 2.9$ & $1.0 \mathrm{~B} 5.2 / 6.5$ \\
\hline $10 \mathrm{~B} 5 / 4$ & .2315 & .3869 & $5 \mathrm{~PB} 5.4 / 4.2$ & 10.0B $5.2 / 4.6$ \\
\hline PB 5/ 4 & .2294 & .3792 & $5 \mathrm{~PB} 5.3 / 4.5$ & 4. $5 \mathrm{~PB} 5.2 / 4.4$ \\
\hline B $5 / 6$ & .2252 & .3735 & $5 \mathrm{~PB} 5.3 / 4.8$ & 5. $0 \mathrm{~B} \quad 5.0 / 6.8$ \\
\hline $10 \mathrm{~PB} 5 / 4$ & .2219 & .3631 & 5 PB $5.3 / 5.3$ & 10.0PB 5. $2 / 5.0$ \\
\hline $10 \mathrm{~B} 5 / 6$ & .2393 & .3615 & $5 \mathrm{~PB} 5.4 / 5.5$ & $9.5 \mathrm{~B} \quad 5.2 / 6.3$ \\
\hline $5 \mathrm{~PB} 5 / 6$ & .2426 & 3539 & $5 \mathrm{~PB} 5.5 / 6.0$ & 4. $0 \mathrm{~PB} 5.3 / 6.2$ \\
\hline PB 5/ 8 & .2606 & .3323 & $5 \mathrm{~PB} 5.6 / 7.6$ & 4. $0 \mathrm{~PB} 5.4 / 7.9$ \\
\hline PB $5 / 10$ & .2567 & .3194 & $5 \mathrm{~PB} 5.6 / 8.5$ & 3. $5 \mathrm{~PB} 5.3 / 8.6$ \\
\hline GY 5/ 8 & .2112 & .7800 & $5 \mathrm{Y} 5.2 / 6.4$ & 5. $0 \mathrm{GY} 5.1 / 7.1$ \\
\hline $10 \mathrm{YR} 5 / 8$ & .1811 & .7859 & $5 Y 4.8 / 6.2$ & $0.5 \mathrm{Y} \quad 5.1 / 7.6$ \\
\hline Y $5 / 6$ & .1831 & .7725 & $5 \mathrm{Y} 4.8 / 5.9$ & $6.0 Y 5.0 / 6.0$ \\
\hline $10 \mathrm{Y} 5 / 6$ & .2155 & .7505 & $5 \mathrm{Y} 5.2 / 5.9$ & $10.0 \mathrm{Y} 5.3 / 5.8$ \\
\hline $10 \mathrm{GY} 5 / 8$ & .2044 & .7160 & $5 \mathrm{Y} 5.1 / 5.2$ & $9.5 \mathrm{GY} 4.9 / 7.8$ \\
\hline GY 5/ 6 & .2076 & .6998 & $5 Y 5.1 / 4.8$ & $5.0 \mathrm{GY} 5.1 / 5.4$ \\
\hline $10 \mathrm{YR} 5 / 6$ & .1873 & .6928 & $5 Y 4.9 / 4.5$ & 10.0YR $5.2 / 5.7$ \\
\hline $10 \mathrm{Y} 5 / 4$ & .2154 & .6766 & $5 \mathrm{Y} 5.2 / 4.4$ & $0.5 \mathrm{GY} 5.3 / 4.4$ \\
\hline Y 5/ 4 & .1871 & .6582 & $5 \mathrm{Y} 4.9 / 3.9$ & $5.0 \mathrm{Y} 5.0 / 3.9$ \\
\hline GY 5/4 & .2069 & .6400 & $5 Y 5.1 / 3.7$ & 5.0GY 5.1/ 4.1 \\
\hline $10 \mathrm{YR} 5 / 4$ & .1854 & .6388 & $5 \mathrm{Y} 4.9 / 3.5$ & 10.0YR $5.1 / 4.4$ \\
\hline $10 \mathrm{GY} 5 / 4$ & .2111 & .5933 & $5 Y 5.2 / 2.8$ & 10.0GY $5.0 / 4.3$ \\
\hline G $5 / 8$ & .2122 & .5890 & $5 Y \quad 5.2 / 2.7$ & 5. $0 \mathrm{G} 4.9 / 8.2$ \\
\hline G $5 / 6$ & .2075 & .5673 & $5 Y 5.1 / 2.3$ & 5. $0 \mathrm{G} 4.9 / 6.6$ \\
\hline G $5 / 4$ & .2008 & .5458 & $5 Y 5.0 / 1.8$ & 5. 0 G $4.9 / 4.8$ \\
\hline GY 5/ 2 & .2097 & .5443 & $5 Y 5.1 / 1.8$ & $5.0 \mathrm{GY} 5.1 / 2.0$ \\
\hline Y $5 / 2$ & .2056 & .5406 & $5 Y 5.1 / 1.7$ & $\begin{array}{llll}4.5 Y & 5.2 / 1.7\end{array}$ \\
\hline YR 5/ 2 & .1813 & .5149 & $5 \mathrm{Y} 4.8 / 1.0$ & 4.0YR $4.9 / 2.1$ \\
\hline G $5 / 2$ & 1971 & .5010 & $5 Y 5.0 / 0.8$ & $6.5 \mathrm{G} \quad 4.9 / 2.4$ \\
\hline R $5 / 2$ & $.1819^{-}$ & .4780 & $5 Y \quad 4.8 / 0.3$ & 4. $0 \mathrm{R} 5.0 / 2.8$ \\
\hline BG $5 / 2$ & .2200 & .4710 & $5 Y \quad 5.2 / 0.1$ & $4.5 \mathrm{BG} 5.1 / 2.5$ \\
\hline $10 \mathrm{RP} 5 / 4$ & .2050 & .4638 & $5 \mathrm{~PB} 5.1 / 0.1$ & 10.1RP $5.3 / 4.2$ \\
\hline N $5 /$ & .1908 & .4610 & $5 \mathrm{~PB} 4.9 / 0.2$ & 5.0PB $4.9 / 0.1$ \\
\hline $10 \mathrm{RP} 5 / 6$ & .1857 & .4588 & $5 \mathrm{~PB} 4.9 / 0.3$ & 10.0RP 5. $2 / 6.6$ \\
\hline RP 5/ 2 & .2048 & .4512 & $5 \mathrm{~PB} 5.1 / 0.7$ & 5.0RP 5. $2 / 2.3$ \\
\hline RP 5/4 & .1943 & .4321 & $5 \mathrm{~PB} 5.0 / 1.5$ & 5.0RP $5.2 / 4.3$ \\
\hline B $5 / 2$ & .2031 & .4244 & $5 \mathrm{~PB} 5.1 / 2.0$ & 6. $5 \mathrm{~B} \quad 4.9 / 2.6$ \\
\hline $\mathrm{RP} 5 / 6$ & .1843 & .4158 & $5 \mathrm{~PB} 4.8 / 2.2$ & 4. $5 \mathrm{RP} 5.1 / 6.2$ \\
\hline P 5/2 & .1941 & .4144 & $5 \mathrm{~PB} 5.0 / 2.4$ & $5.0 \mathrm{P} \quad 5.0 / 2.5$ \\
\hline RP 5/ 8 & .1863 & .4011 & $5 \mathrm{~PB} 4.9 / 2.9$ & 4. $5 \mathrm{RP} 5.2 / 8.0$ \\
\hline PB $5 / 2$ & .2164 & .4042 & $5 \mathrm{~PB} 5.2 / 3.0$ & $5.0 \mathrm{~PB} 5.1 / 3.1$ \\
\hline $10 \mathrm{P} 5 / 4$ & .2005 & .3994 & $5 \mathrm{~PB} 5.0 / 3.2$ & 1.0RP $5.2 / 4.9$ \\
\hline RP 5/10 & .1862 & .3947 & $5 \mathrm{~PB} 4.9 / 3.3$ & 5. $5 \mathrm{RP} 5.3 / 9.7$ \\
\hline B $5 / 4$ & .2187 & .3941 & $5 \mathrm{~PB} 5.2 / 3.6$ & 5. $5 \mathrm{~B} \quad 5.0 / 5.1$ \\
\hline $10 \mathrm{P} 5 / 6$ & .1937 & .3763 & $5 \mathrm{~PB} 5.0 / 4.4$ & 1.0RP 5. $2 / 6.7$ \\
\hline $5 \mathrm{P} 5 / 4$ & .1903 & .3722 & $5 \mathrm{~PB} 4.9 / 4.5$ & 4. $5 \mathrm{P} 5.0 / 4.7$ \\
\hline $10 \mathrm{P} 5 / 8$ & .1933 & .3528 & $5 \mathrm{~PB} 5.0 / 5.6$ & 1.0RP 5. $2 / 8.6$ \\
\hline P 5/6 & .1910 & .3420 & $5 \mathrm{~PB} 4.9 / 6.2$ & 5. 0 P $5.0 / 6.8$ \\
\hline $10 \mathrm{P} 5 / 10$ & .1922 & .3306 & $5 \mathrm{~PB} 4.9 / 7.0$ & $0.5 \mathrm{RP} 5.3 / 10.4$ \\
\hline $10 \mathrm{~PB} 5 / 6$ & .2213 & .3348 & $5 \mathrm{~PB} \quad 5.3 / 7.2$ & 10.0PB 5. $2 / 6.8$ \\
\hline P $5 / 8$ & .2035 & .3137 & $5 \mathrm{~PB} 5.1 / 8.5$ & 5.0P 5.1/8.9 \\
\hline $10 \mathrm{~PB} 5 / 8$ & .2206 & .3073 & 5PB 5. 2/ 9.1 & 10.0PB 5. $2 / 8.4$ \\
\hline $10 \mathrm{~PB} 5 / 10$ & .2104 & .2901 & 5 PB $5.1 / 10.3$ & 10.0PB 5.0/9.6 \\
\hline
\end{tabular}


TABle 5. Protanopic arrangement-Continued

\begin{tabular}{|c|c|c|c|c|}
\hline \multirow{2}{*}{$\begin{array}{l}\text { Munsell } \\
\text { book } \\
\text { notation }\end{array}$} & \multirow{2}{*}{$\begin{array}{c}\text { Protanopic } \\
\text { reflectance, } \\
0.9733 W_{p}\end{array}$} & \multirow{2}{*}{$\begin{array}{l}\text { Protanopic } \\
\text { chroma- } \\
\text { ticity coor- } \\
\text { dinate, } w_{p}\end{array}$} & \multicolumn{2}{|c|}{ Munsell renotations } \\
\hline & & & Protanopic & Normal \\
\hline P $5 / 10$ & 0.1950 & 0.2858 & $5 \mathrm{~PB}$ 5. $0 / 10.4$ & 5. 0 P 5. $1 / 10.9$ \\
\hline YR $5 / 10$ & .1634 & .7770 & $5 \mathrm{Y} \quad 4.6 / 5.8$ & $5.5 \mathrm{YR} 5.1 / 9.0$ \\
\hline YR $5 / 8$ & .1698 & .7196 & $5 Y \quad 4.7 / 4.9$ & 5.5YR 5.1/7.7 \\
\hline $10 \mathrm{R} 5 / 10$ & .1445 & .6865 & $5 \mathrm{Y} 4.4 / 4.1$ & $10.0 \mathrm{R} 5.0 / 9.9$ \\
\hline YR 5/ 6 & .1668 & .6577 & $5 \mathrm{Y} 4.6 / 3.7$ & 5.0YR 5.0/6.2 \\
\hline $10 \mathrm{GY} 4 / 6$ & .1443 & .6487 & $5 \mathrm{Y} 4.4 / 3.4$ & 10.0GY 4.2/5.4 \\
\hline $10 \mathrm{R} 5 / 8$ & .1678 & .6464 & $5 \mathrm{Y} 4.7 / 3.5$ & 0.5 YR $5.2 / 8.7$ \\
\hline $10 \mathrm{R} 5 / 6$ & .1665 & .6023 & $5 Y \quad 4.6 / 2.7$ & $10.0 \mathrm{R} 5.1 / 7.1$ \\
\hline YR 5/ 4 & .1671 & .5925 & $5 Y \quad 4.6 / 2.6$ & 4.5YR $4.9 / 4.6$ \\
\hline $10 \mathrm{GY} 4 / 4$ & .1412 & .5915 & $5 \mathrm{Y} \quad 4.3 / 2.4$ & $1.0 \mathrm{G} \quad 4.2 / 4.1$ \\
\hline Y $4 / 2$ & .1376 & .5526 & $5 Y \quad 4.3 / 1.7$ & 5. $5 \mathrm{Y} \quad 4.3 / 1.7$ \\
\hline G 4/ 4 & .1414 & .5512 & $5 \mathrm{Y} \quad 4.3 / 1.7$ & 5. $0 \mathrm{G} \quad 4.1 / 4.9$ \\
\hline $10 \mathrm{R} 5 / 4$ & .1749 & .5477 & $5 \mathrm{Y} 4.7 / 1.7$ & 9. $5 \mathrm{R} \quad 5.1 / 4.9$ \\
\hline R $5 / 10$ & .1497 & .5437 & $5 Y \quad 4.4 / 1.5$ & 5. 0 R 5. $2 / 10.9$ \\
\hline R $5 / 8$ & .1427 & .5271 & $5 \mathrm{Y} \quad 4.3 / 1.2$ & 4. $5 \mathrm{R}$ 5. $0 / 10.0$ \\
\hline R 5/ 6 & .1572 & .5064 & $5 Y \quad 4.5 / 0.9$ & 4. $0 \mathrm{R} 5.0 / 8.0$ \\
\hline $10 \mathrm{G} 4 / 4$ & .1421 & .5054 & $5 \mathrm{Y} \quad 4.3 / 0.8$ & $0.5 \mathrm{BG} 4.1 / 4.5$ \\
\hline R 5/ 4 & .1636 & .4950 & $5 \mathrm{Y} \quad 4.6 / 0.6$ & $4.0 \mathrm{R} 4.9 / 5.3$ \\
\hline BG $4 / 2$ & .1475 & .4652 & N 4.4/ & 5. $5 \mathrm{BG} 4.3 / 2.5$ \\
\hline BG 4/ 4 & .1561 & .4609 & $5 \mathrm{~PB} \quad 4.5 / 0.1$ & 5. $5 \mathrm{BG} \quad 4.3 / 4.0$ \\
\hline $10 \mathrm{RP} 5 / 8$ & .1665 & .4561 & $5 \mathrm{~PB} \quad 4.6 / 0.4$ & 10.0RP 5. 2/8.6 \\
\hline BG 4/ 6 & .1563 & .4533 & $5 \mathrm{~PB} 4.5 / 0.5$ & 5. $5 \mathrm{BG} 4.2 / 6.0$ \\
\hline $10 \mathrm{RP} 5 / 10$ & .1648 & .4526 & $5 \mathrm{~PB} 4.6 / 0.5$ & 10.0 RP $5.2 / 10.3$ \\
\hline RP 4/ 2 & .1379 & .4425 & $5 \mathrm{~PB} \quad 4.3 / 0.8$ & 4.0RP $4.4 / .2 .1$ \\
\hline $10 \mathrm{BG} 4 / 4$ & .1437 & .4330 & $5 \mathrm{~PB} 4.3 / 1.2$ & $9.5 \mathrm{BG} 4.1 / 4.0$ \\
\hline B 4/ 2 & .1468 & .4230 & $5 \mathrm{~PB} 4.4 / 1.6$ & $5.0 \mathrm{~B} \quad 4.3 / 2.7$ \\
\hline $10 \mathrm{BG} 4 / 6$ & .1564 & 4139 & $5 \mathrm{~PB} 4.5 / 2.0$ & 10.0BG 4.2/5.7 \\
\hline B 4/ 4 & .1619 & .3882 & $5 \mathrm{~PB} 4.6 / 3.2$ & $6.0 \mathrm{~B} 4.4 / 4.6$ \\
\hline $10 \mathrm{~B} 4 / 4$ & .1416 & .3790 & $5 \mathrm{~PB} 4.3 / 3.5$ & $0.5 \mathrm{~PB} 4.2 / 4.1$ \\
\hline PB 4/ 4 & .1444 & .3649 & $5 \mathrm{~PB} 4.4 / 4.3$ & 5.0PB $4.2 / 4.3$ \\
\hline B 4 ! 6 & .1511 & .3561 & $5 \mathrm{~PB} 4.4 / 4.9$ & 5. $5 \mathrm{~B} \quad 4.1 / 6.4$ \\
\hline $5 \mathrm{~B} 4 / 8$ & .1398 & .3433 & $5 \mathrm{~PB} 4.3 / 5.3$ & $6.5 \mathrm{~B} 4.0 / 6.9$ \\
\hline $10 \mathrm{~B} 4 / 6$ & .1505 & .3363 & $5 \mathrm{~PB} 4.4 / 5.9$ & $0.5 \mathrm{~PB} 4.2 / 6.3$ \\
\hline $\mathrm{PB} 4 / 6$ & .1502 & .3339 & $5 \mathrm{~PB} 4.4 / 5.9$ & 4. $5 \mathrm{~PB} 4.2 / 6.0$ \\
\hline $10 \mathrm{~B} 4 / 8$ & .1524 & .3238 & $5 \mathrm{~PB} 4.5 / 6.7$ & $10.0 \mathrm{~B} 4.1 / 7.3$ \\
\hline PB 4/ 8 & .1569 & .3095 & $5 \mathrm{~PB} 4.5 / 7.6$ & 4. $5 \mathrm{~PB} 4.3 / 7.4$ \\
\hline PB $4 / 10$ & .1681 & .2913 & $5 \mathrm{~PB} \quad 4.7 / 9.3$ & 4.0РB $4.4 / 8.9$ \\
\hline YR 4/ 8 & .1114 & .7072 & $5 \mathrm{Y} 3.9 / 4.1$ & 6.0 YR $4.2 / 6.3$ \\
\hline $10 \mathrm{YR} \mathrm{4/4}$ & .1165 & .6860 & $5 Y 3.9 / 3.8$ & 10.0 YR $4.2 / 4.6$ \\
\hline $10 \mathrm{Y} 4 / 4$ & .1340 & .6720 & $5 Y \quad 4.2 / 3.8$ & $10.0 \mathrm{Y} \quad 4.3 / 3.7$ \\
\hline $\mathrm{Y} 4 / 4$ & .1162 & .6784 & $5 Y 3.9 / 3.7$ & $5.0 \mathrm{Y} \quad 4.1 / 3.6$ \\
\hline GY 4/ 6 & .1251 & .6724 & $5 Y 4.1 / 3.7$ & 5.0GY 4.1/ 4.0 \\
\hline YR 4/ 6 & .1203 & .6696 & $5 \mathrm{Y} 4.0 / 3.6$ & 5.0 YR $4.4 / 5.8$ \\
\hline $10 \mathrm{R} 4 / 10$ & .1078 & .6599 & $5 \mathrm{Y} 3.8 / 3.4$ & $9.5 \mathrm{R} 4.4 / 9.0$ \\
\hline $10 \mathrm{R} 4 / 8$ & .1055 & .6514 & $5 Y \quad 3.8 / 3.2$ & $10.0 \mathrm{R} 4.3 / 8.4$ \\
\hline YR 4/ 4 & .1215 & .6265 & $5 Y 4.0 / 2.9$ & 5.0YR $4.3 / 4.6$ \\
\hline GY 4/ 4 & .1316 & .6242 & $5 Y \quad 4.2 / 2.9$ & 5.0GY 4.2/3.2 \\
\hline $10 \mathrm{R} 4 / 6$ & .1102 & .6203 & $5 Y \quad 3.9 / 2.7$ & $10.0 \mathrm{R} 4.3 / 7.3$ \\
\hline $10 \mathrm{R} 4 / 4$ & .1138 & .5661 & $5 Y 3.9 / 1.8$ & 0.5 YR $4.2 / 4.7$ \\
\hline R $5 / 12$ & .1252 & .5638 & $5 Y 4.1 / 1.8$ & $5.5 \mathrm{R} 5.0 / 12.8$ \\
\hline GY 4/ 2 & .1360 & .5429 & $5 \mathrm{Y} 4.2 / 1.5$ & 5. $5 \mathrm{GY} \quad 4.2 / 1.7$ \\
\hline YR 4/ 2 & .1166 & .5343 & $5 \mathrm{Y} 3.9 / 1.2$ & 4.0YR $4.1 / 2.3$ \\
\hline G $4 / 2$ & .1347 & .5075 & $5 Y \quad 4.2 / 0.8$ & $6.0 \mathrm{G} \quad 4.1 / 2.4$ \\
\hline R 4/ 4 & .1046 & .4909 & $5 \mathrm{Y} 3.8 / 0.5$ & $3.5 \mathrm{R} 4.1 / 4.7$ \\
\hline R 4/ 2 & .1180 & .4827 & $5 Y \quad 4.0 / 0.3$ & 4. $0 \mathrm{R} 4.1 / 2.6$ \\
\hline $\mathrm{N} 4 /$ & .1211 & .4597 & $5 \mathrm{~PB} 4.0 / 0.1$ & $5.0 \mathrm{~PB} 4.0 / 0.1$ \\
\hline $10 \mathrm{RP} 4 / 4$ & .1128 & .4513 & $5 \mathrm{~PB} 3.9 / 0.4$ & 9.0RP $4: 1 / 4.1$ \\
\hline
\end{tabular}

TABle 5. Protanopic arrangement-Continued

\begin{tabular}{|c|c|c|c|c|}
\hline \multirow{2}{*}{$\begin{array}{l}\text { Munsell } \\
\text { book } \\
\text { notation }\end{array}$} & \multirow{2}{*}{$\begin{array}{l}\text { Protanopic } \\
\text { reflectance, } \\
0.9733 W_{p}\end{array}$} & \multirow{2}{*}{$\begin{array}{c}\text { Protanopic } \\
\text { chroma- } \\
\text { ticity coor- } \\
\text { dinate, } w_{p}\end{array}$} & \multicolumn{2}{|c|}{ Munsell renotations } \\
\hline & & & Protanopic & Normal \\
\hline RP 4/4 & 0.1282 & 0.4152 & $5 \mathrm{~PB} 4.1 / 1.8$ & 4.0RP $4.3 / 4.4$ \\
\hline P $4 / 2$ & .1234 & .4067 & $5 \mathrm{~PB} 4.1 / 2.2$ & 5.0P 4.1/2.2 \\
\hline PB $4 / 2$ & .1359 & .4035 & $5 \mathrm{~PB} 4.2 / 2.4$ & 5. $0 \mathrm{~PB} 4.2 / 2.5$ \\
\hline RP 4/ 6 & .1206 & .3925 & $5 \mathrm{~PB} 4.0 / 2.7$ & 4. $5 \mathrm{RP} 4.3 / 6.4$ \\
\hline $10 \mathrm{P} 4 / 4$ & .1329 & .3861 & $5 \mathrm{~PB} 4.2 / 3.1$ & $0.5 \mathrm{RP} 4.3 / 4.5$ \\
\hline RP 4/ 8 & .1098 & .3759 & $5 \mathrm{~PB} 3.8 / 3.1$ & 4. $5 \mathrm{RP} 4.2 / 7.9$ \\
\hline RP $4 / 10$ & .1060 & .3602 & $5 \mathrm{~PB} 3.8 / 3.8$ & 4. $5 \mathrm{RP} 4.2 / 9.4$ \\
\hline P $4 / 4$ & .1256 & .3654 & $5 \mathrm{~PB} 4.1 / 4.0$ & 5.0P $4.1 / 4.2$ \\
\hline RP $4 / 12$ & .1047 & .3465 & $5 \mathrm{~PB} 3.8 / 4.5$ & 5. 0 RP $4.3 / 10.7$ \\
\hline $10 \mathrm{P} 4 / 6$ & .1262 & .3530 & 5PB 4.1/4.6 & $0.5 \mathrm{RP} 4.3 / 6.6$ \\
\hline $10 \mathrm{~PB} 4 / 4$ & .1314 & .3483 & $5 \mathrm{~PB} 4.2 / 5.0$ & 10.0PB 4.1/4.6 \\
\hline P $4 / 6$ & .1198 & .3297 & $5 \mathrm{BP} 4.0 / 5.8$ & 5.0P $4.1 / 6.1$ \\
\hline $10 \mathrm{P} 4 / 8$ & .1226 & .3274 & $5 \mathrm{~PB} 4.0 / 5.9$ & $0.5 \mathrm{RP} 4.3 / 8.5$ \\
\hline $10 \mathrm{~PB} 4 / 6$ & .1365 & .3111 & $5 \mathrm{~PB} 4.2 / 7.1$ & 10.0PB 4. $2 / 6.6$ \\
\hline P $4 / 8$ & .1219 & .2977 & $5 \mathrm{~PB} 4.0 / 7.8$ & 5.0P 4.1/ 8.2 \\
\hline $10 \mathrm{P} 4 / 10$ & .1112 & .2964 & $5 \mathrm{~PB} 3.9 / 7.5$ & 0.5RP 4. 2/10.4 \\
\hline $10 \mathrm{~PB} 4 / 8$ & .1347 & .2824 & $5 \mathrm{~PB} 4.2 / 9.2$ & 10.0PB 4.1/8.5 \\
\hline $5 \mathrm{P} 4 / 10$ & .1212 & .2754 & $5 \mathrm{~PB} 4.0 / 9.3$ & 5. $0 \mathrm{P} 4.1 / 10.1$ \\
\hline $10 \mathrm{~PB} \quad 4 / 10$ & .1283 & .2633 & 5PB 4.1/10.2 & $10.0 \mathrm{~PB} 4.0 / 9.7$ \\
\hline P $4 / 12$ & .1145 & .2388 & 5 P B $3.9 / 12.1$ & 5. $5 \mathrm{P} 4.1 / 12.6$ \\
\hline
\end{tabular}

$10 \mathrm{GY} 3 / 4$

G $3 / 4$

$10 \mathrm{Y} 3 / 2$

R $4 / 12$

R $4 / 10$

R 4/8

R 4/ 6

10G $3 / 4$

$5 \mathrm{BG} 3 / 2$

BG $3 / 4$

10RP $4 / 6$

BG $3 / 6$

10RP 4/ 8

10RP $4 / 10$

$10 \mathrm{BG} 3 / 4$

B $3 / 2$

10BG $3 / 6$

B $3 / 4$

$10 \mathrm{~B} 3 / 4$

B $3 / 6$

10B $3 / 6$

PB 3/ 6

10B $3 / 8$

PB $3 / 8$

PB $3 / 10$

PB $3 / 12$

YR 3/ 4

10YR 3/ 2

GY 3/ 4

R 4/14

R $4 / 14$

$10 \mathrm{R} 3 / 6$

$5 \mathrm{GY} 3 / 2$

10R 3/4

$\mathrm{YR} 3 / 2$

YR 3/ 2

Y $3 / 2$

G $3 / 2$

R 3/4

R $3 / 6$

R 3/ 2

N $3 /$
$5 Y 3.3 / 2.8$ 5Y 3. $5 / 1.8$

$5 \mathrm{Y} 3.3 / 1.8$

5Y 3. 3/ 1.8

$5 Y 3.4 / 1.2$

$5 \mathrm{Y} \quad 3.5 / 1.1$ $5 Y 3.6 / 0.8$

5 Y $3.4 / 0.8$ $5 \mathrm{~PB} 3.3 / 0.1$ 5PB 3.3/ 0.3

5PB 3.7/0.6 $5 \mathrm{~PB} 3.5 / 0.7$ $5 \mathrm{~PB} 3.7 / 1.1$ 5PB 3.6/ 1.1 5PB 3.5/1.4

$5 \mathrm{~PB} 3.3 / 1.5$ 5PB 3.4/ 2.1 $5 \mathrm{~PB} 3.3 / 2.6$ $\begin{array}{lll}5 \mathrm{~PB} & 3.3 / 3.4\end{array}$ 5PB 3.6/3.9

5 PB 3.5/5.2 $5 \mathrm{~PB} 3.4 / 5.8$ $5 \mathrm{~PB} 3.7 / 6.7$ $5 \mathrm{~PB} 3.5 / 7.3$ $5 \mathrm{~PB} 3.7 / 9.0$ 5PB 3.6/9.5

$5 Y 3.0 / 2.6$ $5 \mathrm{Y} 3.0 / 2.3$ $5 \mathrm{Y} 3.2 / 2.3$

5Y 3. $2 / 2.1$

$5 Y 3.2 / 2.1$ $5 Y$ 2.9/2.0

$5 \mathrm{Y} 3.2 / 1.6$ $5 Y$ 2.9/1.4

$5 Y 3.0 / 1.3$

$5 \mathrm{Y} 3.0 / 1.3$

$5 \mathrm{Y} 3.0 / 1.2$

$5 \mathrm{Y} 3.2 / 1.0$ 5 Y 2. 9/ 0.6

$\begin{array}{lll}5 \mathrm{Y} & 2.9 / 0.6\end{array}$

$5 \mathrm{Y} \quad 3.0 / 0.2$ 5PB 3.0/ 0.2
$10.0 \mathrm{GY} 3.2 / 4.5$ 4. 0 G $3.3 / 4.8$ 0. $5 \mathrm{GY} 3.3 / 1.8$ 5. $0 \mathrm{R} 4.2 / 12.6$ 4. 5 R $4.2 / 10.8$

4. 5R 4. 2/ 9.4 4. $5 \mathrm{R} 4.1 / 6.8$ 10.0G 3. $2 / 4.7$ 5. 0 BG 3. $2 / 2.8$ 5. 0 BG 3.1/ 4.1

9. 5 RP $4.1 / 6.4$ 6. $0 \mathrm{BG} 3.2 / 5.3$ 9. 0RP 4. 2/ 8.2 9. 5RP 4. 2/ 9.5 9. $5 \mathrm{BG} 3.3 / 4.3$

5. 5B 3. 1/ 2.3 9. $5 \mathrm{BG} 3.2 / 5.8$ 4. 5B 3.1/ 4.2 9. 5 B $3.1 / 3.9$ 5. 0 B $3.3 / 5.9$

9.5B $3.2 / 5.6$ 5. 0 PB $3.2 / 5.6$ 9. $0 \mathrm{~B} 3.3 / 7.1$ 4. $5 \mathrm{~PB} 3.2 / 7.0$ 4. $5 \mathrm{~PB} \mathrm{3.4/8.5}$ 4. 0 PB 3. $3 / 9.4$

5. 0 YR $3.3 / 4.1$ 10. 0 Y R 3. $1 / 2.7$ 5. 0 GY $3.2 / 2.6$ 5. $5 \mathrm{R}$ 4. $2 / 13.2$ 5. $5 \mathrm{R}$ 4. $2 / 13.2$ 10.0R 3. $3 / 5.4$ 4. $5 \mathrm{GY} 3.2 / 1.9$ 10. $0 \mathrm{R} 3.2 / 4.1$ 5. OYR 3.1/2.1 5. 0 Y R 3.1/2.0 6. 0 Y 3.1/ 1.1

5. 0 G $3.1 / 2.7$ 5. 0 R 3.2/4.6 4. 5 R $3.4 / 6.2$ 3. $5 \mathrm{R} 3.1 / 2.1$ 2. $5 \mathrm{P} 3.0 / 0.2$ 
TABLE 5. Protanopic arrangement-Continued

\begin{tabular}{|c|c|c|c|c|c|}
\hline \multirow{2}{*}{$\begin{array}{l}\text { Munsell } \\
\text { book } \\
\text { notation }\end{array}$} & \multirow{2}{*}{$\begin{array}{c}\text { Protanopic } \\
\text { reflectance, } \\
0.9733 \mathrm{~W}_{p}\end{array}$} & \multirow{2}{*}{$\begin{array}{l}\text { Protanopic } \\
\text { chroma- } \\
\text { ticity coor- } \\
\text { dinate, } w_{p}\end{array}$} & \multicolumn{2}{|c|}{ Munsell renotations } & . \\
\hline & & & Protanopic & Normal & \\
\hline 10RP $3 / 4$ & 0.0569 & 0.4412 & $5 \mathrm{~PB} 2.8 / 0.5$ & $9.0 \mathrm{RP} 3.0 /$ & 3.4 \\
\hline RP 3/ 2 & .0611 & .4304 & $5 \mathrm{~PB} 2.9 / 0.8$ & 4. $5 \mathrm{RP} 3.0 /$ & 1.9 \\
\hline $10 \mathrm{RP} 3 / 6$ & .0562 & .4300 & 5PB 2.8/ 0.8 & 9.0RP 3.1/ & 5. 6 \\
\hline $\mathrm{RP} 3 / 4$ & .0590 & .4037 & $5 \mathrm{~PB} 2.8 / 1.4$ & 5. $0 \mathrm{RP} 3.0 /$ & 3. 7 \\
\hline P $3 / 2$ & .0722 & .3889 & $5 \mathrm{~PB} 3.1 / 2.1$ & $5.0 \mathrm{P} 3.2 /$ & 2. 2 \\
\hline PB 3/ 2 & .0719 & .3875 & $5 \mathrm{~PB} 3.1 / 2.1$ & 5. $0 \mathrm{~PB} \mathrm{3.1/}$ & 2.1 \\
\hline RP $3 / 8$ & .0551 & .3540 & $5 \mathrm{~PB} 2.7 / 3.0$ & 5. $0 \mathrm{RP} 3.1 /$ & 6. 8 \\
\hline $10 \mathrm{P} 3 / 4$ & .0665 & .3543 & 5 PB $3.0 / 3.2$ & $10.0 \mathrm{P} 3.2 /$ & 4.5 \\
\hline $\mathrm{RP} 3 / 10$ & .0553 & .3353 & 5 PB $2.8 / 3.7$ & 5. $0 \mathrm{RP} 3.2 /$ & 8.4 \\
\hline PB 3/ 4 & .0757 & .3415 & $5 \mathrm{~PB} 3.2 / 3.9$ & 5. 0 PB $3.1 /$ & 3.9 \\
\hline P $3 / 4$ & .0679 & .3256 & 5PB $3.1 / 4.2$ & $5.0 \mathrm{P} 3.1 /$ & 4.5 \\
\hline $10 \mathrm{P} 3 / 6$ & .0645 & .3141 & 5 PB $3.0 / 4.9$ & 9. $0 \mathrm{P} 3.2 /$ & 6.3 \\
\hline $10 \mathrm{~PB} 3 / 4$ & .0728 & .3128 & $5 \mathrm{~PB} 3.2 / 5.3$ & 10.0PB 3.1/ & 4. 7 \\
\hline P $3 / 6$ & .0711 & 2914 & 5 PB $3.1 / 6.2$ & 4. $5 \mathrm{P} 3.2 /$ & 6.4 \\
\hline $10 \mathrm{~PB} 3 / 6$ & .0730 & .2858 & 5 PB 3.2/6.7 & 10.0PB 3.1/ & 6.1 \\
\hline $10 \mathrm{P} 3 / 8$ & .0589 & .2794 & 5РB $2.8 / 6.5$ & $10.0 \mathrm{P} 3.1 /$ & 8.3 \\
\hline P $3 / 8$ & .0712 & .2611 & $5 \mathrm{~PB} 3.1 / 8.1$ & $5.0 \mathrm{P} 3.2 /$ & 8.4 \\
\hline $10 \mathrm{~PB} 3 / 8$ & .0749 & .2643 & $5 \mathrm{~PB} 3.2 / 8.2$ & 10.0PB $3.2 /$ & 7.4 \\
\hline P $3 / 10$ & .0700 & .2224 & $5 \mathrm{~PB} 3.1 / 11.0$ & 5. $0 \mathrm{P} 3.3 / 1$ & 11. 2 \\
\hline $10 \mathrm{~PB} 3 / 10$ & .0735 & .2098 & $5 \mathrm{~PB} 3.2 / 11.8$ & 10. 0 PB $3.1 / 1$ & 10.6 \\
\hline GY 2/ 2 & .0465 & .5684 & $5 \mathrm{Y} 2.5 / 1.6$ & 5.5GY $2.5 /$ & 2. 2 \\
\hline $10 \mathrm{Y} 2 / 2$ & .0475 & .5609 & $5 \mathrm{Y} 2.5 / 1.5$ & $10.0 \mathrm{Y} 2.6 /$ & 1.6 \\
\hline $\mathrm{Y} 2 / 2$ & .0455 & .5553 & $5 \mathrm{Y} 2.5 / 1.4$ & $5.0 \mathrm{Y} 2.5 /$ & 1.4 \\
\hline $10 \mathrm{GY} 2 / 2$ & .0456 & .5552 & $5 \mathrm{Y} 2.5 / 1.4$ & 9. 5GY 2.4/ & 2.5 \\
\hline $10 \mathrm{YR} 2 / 2$ & .0472 & .5419 & $5 \mathrm{Y} 2.5 / 1.2$ & 7.5YR 2.6/ & 1.6 \\
\hline R $3 / 10$ & .0487 & .5371 & $5 Y 2.6 / 1.1$ & $5.0 \mathrm{R} 3.2 /$ & 8.3 \\
\hline YR 2/ 2 & .0403 & .5274 & $5 \mathrm{Y} 2.3 / 0.9$ & 4. 5YR 2.4/ & 1.8 \\
\hline R $3 / 8$ & .0519 & .5241 & $5 Y 2.7 / 0.9$ & 5. $0 \mathrm{R} 3.2 /$ & 7.4 \\
\hline G $2 / 2$ & .0398 & .5081 & $5 \mathrm{Y} 2.3 / 0.6$ & $7.0 \mathrm{G} 2.2 /$ & 2.4 \\
\hline $10 \mathrm{R} 2 / 2$ & .0397 & .5056 & $5 Y 2.3 / 0.6$ & 8.5R 2.5/ & 2.6 \\
\hline $10 \mathrm{G} 2 / 2$ & .0445 & .4967 & $5 \mathrm{Y} 2.5 / 0.4$ & $10.0 \mathrm{G} 2.3 /$ & 3.1 \\
\hline $5 \mathrm{BG} 2 / 2$ & .0412 & .4623 & $5 \mathrm{~PB} 2.3 / 0.1$ & 4. $5 \mathrm{BG} 2.2 /$ & 2.5 \\
\hline $\mathrm{BG} 2 / 4$ & .0446 & .4544 & $5 \mathrm{~PB} 2.5 / 0.2$ & 5. $0 \mathrm{BG} 2.3 /$ & 3. 7 . \\
\hline $10 \mathrm{BG} 2 / 2$ & 0507 & .4400 & $5 \mathrm{~PB} 2.6 / 0.5$ & 7.0BG 2.5/ & 3.1 \\
\hline B $2 / 2$ & .0414 & .4216 & $5 \mathrm{~PB} 2.4 / 0.9$ & $2.0 \mathrm{~B} 2.2 /$ & 2.2 \\
\hline $10 \mathrm{RP} 3 / 8$ & .0516 & .4167 & $5 \mathrm{~PB} 2.7 / 1.0$ & 9.0RP 3.1/ & 7.0 \\
\hline RP 3/ 6 & .0549 & .3837 & $5 \mathrm{~PB} 2.7 / 2.0$ & 5.0RP $3.0 /$ & 5. 2 \\
\hline $10 \mathrm{RP} 3 / 10$ & .0508 & .3807 & 5 PB $2.6 / 2.0$ & 8.5RP $3.2 /$ & 8.5 \\
\hline 10B 2/ 2 & .0435 & .3664 & $5 \mathrm{~PB} 2.4 / 2.4$ & 8. 5B 2.2/ & 3.1 \\
\hline PB 2/ 4 & .0437 & .3030 & 5PB 2.4/ 4.7 & 4. $0 \mathrm{~PB} 2.3 /$ & 4. 8 \\
\hline $10 \mathrm{~PB} 2 / 4$ & .0391 & .2884 & $5 \mathrm{~PB} 2.3 / 5.1$ & $10.0 \mathrm{~PB} \mathrm{2.2/}$ & 4.8 \\
\hline PB 2/ 6 & .0474 & .2802 & $5 \mathrm{~PB} 2.5 / 5.8$ & 4.0PB 2.3/ & 5.9 \\
\hline $10 \mathrm{P} 3 / 10$ & .0531 & .2438 & $5 \mathrm{~PB} 2.7 / 8.3$ & $9.0 \mathrm{P} 3.0 /$ & 9.8 \\
\hline R 2/ 4 & .0311 & .4713 & $5 \mathrm{Y} 2.0 / 0.1$ & 4. $0 \mathrm{R} 2.3 /$ & 3.8 \\
\hline $\mathrm{R} 2 / 2$ & .0357 & .4663 & $5 Y 2.2 / 0.05$ & $3.5 \mathrm{R} 2.3 /$ & 2.2 \\
\hline R 2/ 6 & .0359 & .4595 & $5 \mathrm{~PB} 2.0 / 0.1$ & $3.0 \mathrm{R} 2.5 /$ & 4.8 \\
\hline N 2/ & .0300 & .4570 & $5 \mathrm{~PB} 2.0 / 0.1$ & $5.0 \mathrm{P} 2.0 /$ & 0.2 \\
\hline RP 2/ 2 & .0352 & .4204 & 5 PB 2.1/0.9 & 6.0RP 2.3/ & 2.3 \\
\hline $10 \mathrm{RP} 2 / 4$ & .0336 & .4127 & $5 \mathrm{~PB} 2.1 / 1.1$ & 8.0RP 2.3/ & 3. 7 \\
\hline 10RP $2 / 6$ & .0323 & .3790 & $5 \mathrm{~PB} 2.0 / 1.9$ & 8.0RP 2.4/ & 5.6 \\
\hline RP 2/ 4 & .0345 & .3691 & 5РB 2.1/ 2.2 & 5.0RP 2.4/ & 4. 6 \\
\hline PB 2/ 2 & .0378 & .3603 & $5 \mathrm{~PB} 2.2 / 2.5$ & 4. 5PB 2. 2/ & 2.6 \\
\hline P 2/ 2 & .0340 & .3493 & $5 \mathrm{~PB} 2.1 / 2.8$ & 5. 5 P 2.1/ & 2.9 \\
\hline RP 2/ 6 & .0359 & .3385 & 5PB 2. 2/ 3.1 & 4.0RP 2.4/ & 5.9 \\
\hline $\mathrm{P} 2 / 4$ & .0344 & .3072 & 5PB 2.1/4.2 & 5.0P 2.2/ & 4. 6 \\
\hline $10 \mathrm{P} 2 / 4$ & .0333 & .3051 & 5PB 2.1/ 4.3 & 9.0P 2.2/ & 5.3 \\
\hline $10 \mathrm{~PB} 2 / 6$ & .0368 & .2767 & 5PB 2.2/5. 6 & $10.0 \mathrm{~PB} 2.2 /$ & 5.5 \\
\hline $10 \mathrm{P} 2 / 6$ & .0327 & .2723 & 5PB 2.1/5.6 & 9. $0 \mathrm{P} 2.2 /$ & 6.8 \\
\hline P 2/ 6 & .0323 & .2580 & $5 \mathrm{~PB} 2.0 / 6.2$ & $5.0 \mathrm{P} 2.1 /$ & 6.8 \\
\hline
\end{tabular}

Thus far no precise indication has been given of the color perceptions of deuteranopes and protanopes corresponding to the 400 samples in the Munsell Book of Color; we have only grouped together those samples that differ least and that are therefore most likely to be confused. We know, however, that deuteranopes and protanopes in all probability perceive black, gray, and white normally; furthermore, we know (see table 3) that their yellow corresponds closely to what the normal observer sees in the spectrum at $575 \mathrm{~m} \mu$, and their blue corresponds closely to $470 \mathrm{~m} \mu$. These facts may be conveniently expressed in the Munsell color system by giving for each set of values of $W / W_{0}$ and $w$ the Munsell equivalent of the purple-blue $(P B)$ or yellow $(Y)$ hue; see master hue chart, figure 13 of the OSA Subcommittee on the Spacing of the Munsell Colors [76]. These Munsell hues correspond closely to those of the spectrum at 470 and $575 \mathrm{~m} \mu$, respectively, and since the exact hues corresponding to these portions of the spectrum depend somewhat on the observing conditions $[14,17,96]$ more precise fitting (as by $6 P B$ and $7 Y$ ) is probably not warranted.

The deuteranopic and protanopic Munsell renotations based in this way resulted from the following steps: (a) from table 1 of the OSA Subcommittee Report [76] (pp. 399 and 402) were read the chromaticity coordinates $(x, y)$ for enough colors of the hues $5 \mathrm{Y}$ and $5 \mathrm{~PB}$ to cover the range of the Munsell papers; (b) from these chromaticity coordinates were computed the dichromatic coordinates $w_{d}$ and $w_{p}$, according to eq $2 \mathrm{~d}$ and $2 \mathrm{p}$ and these coordinates were tabulated in table 6 ; (c) from the data of table 6 families of curves were plotted on each of two graphs, one with $w_{d}$ as the abscissa, the other with $w_{p}$ as the abscissa, the ordinate being Munsell chroma and each curve showed the variation of Munsell chroma for hue $5 \mathrm{Y}$ or $5 \mathrm{~PB}$ with dichromatic coordinate $\left(w_{d}\right.$ or $\left.w_{p}\right)$ for some one Munsell value; (d) from the reflectance relative to magnesium oxide, $W / W_{0}$, (second col of tables 4 and 5 ), the Munsell value was read from table 2 of the subcommittee report [76]; and (e) by interpolation among the curves of the corresponding family according to this Munsell value, the Munsell hue (whether $5 \mathrm{Y}$ or $5 \mathrm{~PB}$ ) was determined, and the Munsell chroma corresponding to the dichromatic coordinate, $w_{d}$ or $w_{p}$, was read with an uncertainty of about 0.1 chroma step. The Munsell di- 
chromatic renotations (Hue Value/Chroma) so found are given in the fourth column of tables 4 and 5. The fifth column is the normal Munsell renotation copied from table 3 of the subcommittee report [76].

Comparison of these two columns (dichromatic Munsell renotation with normal Munsell renotation) indicates in a precise and detailed way the difference between the surface-color perceptions of the normal observer and those of the average deuteranope and average protanope, respectively.

TABLE: 6. Deuteranopic and protanopic chromaticity coordinates for Munsell renotations of hues $5 Y$ and $5 P B$

\begin{tabular}{|c|c|c|c|c|c|}
\hline \multirow{2}{*}{$\begin{array}{c}\text { Munsell } \\
\text { renotation }\end{array}$} & \multicolumn{2}{|c|}{$\begin{array}{l}\text { Chromaticity } \\
\text { coordinates }\end{array}$} & \multirow{2}{*}{$\begin{array}{l}\text { Munsell } \\
\text { renotation }\end{array}$} & \multicolumn{2}{|c|}{$\begin{array}{l}\text { Chromaticity } \\
\text { coordinates }\end{array}$} \\
\hline & $w_{d}$ & $w_{p}$ & & $w_{d}$ & $w_{p}$ \\
\hline $5 \mathrm{Y} 9 / 12$ & 0.8510 & 0.8433 & $5 \mathrm{~PB} 8 / 6$ & 0.3615 & 0.3810 \\
\hline $5 Y 9 / 10$ & .7911 & .7827 & $5 \mathrm{~PB} 8 / 4$ & .3973 & .4119 \\
\hline $5 Y 9 / 8$ & .7296 & .7218 & $5 \mathrm{~PB} 8 / 2$ & .4325 & .4425 \\
\hline $5 Y 9 / 6$ & .6628 & .6569 & & & \\
\hline $5 \mathrm{Y} 9 / 4$ & .5955 & .5929 & $5 \mathrm{~PB} \quad 7 / 10$ & .2927 & .3214 \\
\hline \multirow[t]{2}{*}{$5 Y 9 / 2$} & .5291 & .5305 & $5 \mathrm{~PB} 7 / 8$ & .3246 & .3489 \\
\hline & & & $5 \mathrm{~PB} 7 / 6$ & .3570 & .3768 \\
\hline $5 \mathrm{Y} 8 / 12$ & .8805 & .8731 & $5 \mathrm{~PB} 7 / 4$ & .3913 & .4065 \\
\hline $5 Y 8 / 10$ & .8181 & .8093 & $5 \mathrm{~PB} 7 / 2$ & .4272 & .4377 \\
\hline $5 \mathrm{Y} 8 / 8$ & .7494 & .7408 & & & \\
\hline $5 Y 8 / 6$ & .6764 & .6696 & $5 \mathrm{~PB} 6 / 12$ & .2507 & .2856 \\
\hline $5 \mathrm{Y} 8 / 4$ & .6025 & .5993 & $5 \mathrm{~PB} 6 / 10$ & .2804 & .3107 \\
\hline $5 \mathrm{Y} 8 / 2$ & .5325 & .5335 & $5 \mathrm{~PB} 6 / 8$ & $\begin{array}{l}.200 t \\
.3096\end{array}$ & .3354 \\
\hline $5 Y \quad 7 / 12$ & .9125 & .9061 & $5 \mathrm{~PB} 6 / 6$ & .3426 & .3638 \\
\hline $5 \mathrm{Y} 7 / 10$ & .8552 & $\begin{array}{l}.8001 \\
.8466\end{array}$ & $5 \mathrm{~PB} 6 / 4$ & .3823 & .3984 \\
\hline $5 \mathrm{Y} 7 / 8$ & .7788 & $\begin{array}{l}.0400 \\
.7693\end{array}$ & 5 PB $6 / 2$ & .4208 & .4319 \\
\hline $5 Y 7 / 6$ & .7007 & .6927 & $5 \mathrm{~PB} 5 / 12$ & .2299 & .2675 \\
\hline $5 Y 7 / 4$ & .6184 & .6139 & $5 \mathrm{~PB} 5 / 10$ & .2577 & .2909 \\
\hline \multirow[t]{2}{*}{$5 Y 7 / 2$} & .5379 & .5383 & $5 \mathrm{~PB} 5 / 8$ & .2891 & .3176 \\
\hline & 9425 & 9377 & $5 \mathrm{~PB} 5 / 6$ & .3242 & .3476 \\
\hline $\begin{array}{lll}5 Y & 0 / 12 \\
5 Y & 6 / 10\end{array}$ & $\begin{array}{l}.9425 \\
.8935\end{array}$ & $\begin{array}{l}.9377 \\
.8859\end{array}$ & $5 \mathrm{~PB} 5 / 4$ & .3662 & .3838 \\
\hline $5 \mathrm{Y} 6 / 8$ & $\begin{array}{l}.0900 \\
.8231\end{array}$ & $\begin{array}{l}.8859 \\
.8134\end{array}$ & $5 \mathrm{~PB} 5 / 2$ & .4106 & .4226 \\
\hline $5 \mathrm{Y} 6 / 6$ & .7346 & .7251 & $5 \mathrm{~PB} 4 / 12$ & .2017 & .2426 \\
\hline $5 \mathrm{Y} 6 / 4$ & .6373 & .6314 & $5 \mathrm{~PB} 4 / 10$ & .2282 & .2653 \\
\hline $5 \mathrm{Y} 6 / 2$ & .5471 & .5468 & $5 \mathrm{~PB} 4 / 8$ & $\begin{array}{r}.2202 \\
.2596\end{array}$ & .2919 \\
\hline \multirow{2}{*}{$5 Y \quad 5 / 12$} & & & $5 \mathrm{~PB} 4 / 6$ & .2997 & .3261 \\
\hline & .9903 & .9894 & $5 \mathrm{~PB} 4 / 4$ & .3442 & .3642 \\
\hline $\begin{array}{ll}5 \mathrm{Y} & 5 / 10 \\
5 \mathrm{Y} & 5 / 8\end{array}$ & .9336 & .9279 & $5 \mathrm{~PB} 4 / 2$ & .3956 & .4091 \\
\hline $\begin{array}{ll}5 Y & 5 / 8 \\
5 Y & 5 / 6\end{array}$ & $\begin{array}{r}.8655 \\
.7783\end{array}$ & $\begin{array}{r}.8562 \\
7677\end{array}$ & & & \\
\hline $5 \mathrm{Y} 5 / 4$ & 6667 & .6588 & $5 \mathrm{~PB} \quad 3 / 12$ & .1606 & .2065 \\
\hline \multirow{2}{*}{$5 Y 5 / 2$} & $\begin{array}{l}.0008 \\
5569\end{array}$ & $\begin{array}{r}.6588 \\
5556\end{array}$ & $5 \mathrm{~PB} \quad 3 / 10$ & .1886 & .2305 \\
\hline & .5569 & .5556 & $5 \mathrm{~PB} 3 / 8$ & .2223 & .2594 \\
\hline $5 \mathrm{Y} 4 / 8$ & .9153 & .9081 & $5 \mathrm{~PB} 3 / 6$ & .2605 & .2919 \\
\hline $5 \mathrm{Y} 4 / 6$ & .8200 & $\begin{array}{l}.8001 \\
.8092\end{array}$ & $5 \mathrm{~PB} 3 / 4$ & .3104 & .3346 \\
\hline $5 Y 4 / 4$ & .7061 & $\begin{array}{l}.8092 \\
.6962\end{array}$ & $5 \mathrm{~PB} 3 / 2$ & .3729 & .3890 \\
\hline \multirow[t]{2}{*}{$5 Y 4 / 2$} & .5774 & .5741 & $5 \mathrm{~PB} 2 / 12$ & .1213 & .1709 \\
\hline & & & $5 \mathrm{~PB} \quad 2 / 10$ & .1459 & .1925 \\
\hline $5 \mathrm{Y} 3 / 6$ & .8839 & .8745 & $5 \mathrm{~PB} 2 / 8$ & .1793 & .2215 \\
\hline $5 Y 3 / 4$ & .7373 & .7261 & $5 \mathrm{~PB} 2 / 6$ & 2247 & 2604 \\
\hline $5 \mathrm{Y} 3 / 2$ & .5899 & .5854 & $5 \mathrm{~PB} 2 / 4$ & .2833 & .3107 \\
\hline \multirow{2}{*}{$\begin{array}{lll}5 \mathrm{Y} & 2 / 4 \\
5 \mathrm{Y} & 2 / 2\end{array}$} & .8380 & .8266 & $5 \mathrm{~PB} 2 / 2$ & .3564 & .3740 \\
\hline & .6149 & .6182 & $5 \mathrm{~PB} \quad 1 / 10$ & .0998 & .1506 \\
\hline \multirow[t]{2}{*}{$5 Y 1 / 2$} & .7392 & .7267 & $5 \mathrm{~PB} 1 / 8$ & .1314 & .1785 \\
\hline & & & $5 \mathrm{~PB} 1 / 6$ & .1739 & .2157 \\
\hline $\mathrm{N} 1 /$ to $9 /$ & .4585 & .4652 & $5 \mathrm{~PB} 1 / 4$ & .2337 & .2671 \\
\hline $5 \mathrm{~PB} 9 / 2$ & .4362 & .4457 & $5 \mathrm{~PB} 1 / 2$ & .3127 & .3355 \\
\hline
\end{tabular}

It will be noted, of course, first that the normal observer perceives a multiplicity of hues (red, yellow-red, yellow, green-yellow, green, and so on), while the deuteranope and protanope perceive but two-Munsell yellow and Munsell purple-blue. Second, it will be noted from table 4 that the Munsell value of each sample for the deuterảnope is the same as that for the normal observer, but from table 5 the protanopic and normal Munsell value differs except for hues near to yellow-green ( $3 \mathrm{GY}$ ) and bluish purple (2P). The reddish colors have lower protanopic values, and the greenish have higher; the amount of the difference from the normal values varying from one value step for R 4/14 to zero for nearly neutral colors.

Table 5 also serves to indicate the degree to which protanopic vision leads to confusions between colors easily distinguishable by a normal observer. For samples of small size, such as those that yield ideal protanopic vision, a difference of 0.1 -value step or 0.2 -chroma step is so small as to be easily confusible with zero difference. From table 5 it may be seen therefore, that Munsell samples GY 8/6 and 10 Y 8/6 would be confusible to a typical dichromat if viewed in small size, though to the normal observer, of course, these two samples are distinctly different. By proceeding along the fourth column of table 5 and noting the samples whose protanopic renotations differ by 0.1 or less in value and at the same time by 0.2 or less in chroma, we may make an estimate of the frequency with which a protanope will be troubled to distinguish colors that to the normal observer are separated by at least 10 just noticeable steps. It is found that there are 97 such pairs among the 410 different colors listed; that is, in a group of colors chosen in such a way as to be unrelated to the characteristic protanopic confusions, still about one-fourth of the colors are by chance confusible with another of the group. ${ }^{3}$ This result may explain, on the one hand, why dichromatic observers often reach maturity without having become convinced that there is anything abnormal about them other than unusual unfamiliarity with the meaning of color terms, since in about threefourths of the pairs that they are called upon to

\footnotetext{
${ }^{3}$ A similar count of pairs confusible by the deuteranope can be made from the fourth column of table 4 , and about 200 will be found, roughly twice as many, as from table 5. This is ascribable to the fact that the samples are not unrelated to deuteranopic confusions, being divisible into seven groups, each composed of samples differing but slightly in deuteranopic Munsell value.
} 
compare, they see a difference but describe it in terms not used by the normal observer. On the other hand, since one-fourth of the differences that the normal observer sees plainly are so slight to the red-green confusing dichromat that he must look long and closely to decide about them, and occasionally fails completely, it is easy to understand that a characteristic by which the dichromat is marked is his tendency to hesitate before making a decision, to wait for someone else to speak up, and then to agree with him.

It is suggested that persons who have been found to have either protanopic or deuteranopic vision by the usual tests might find it interesting and possibly instructive to lay out the Munsell papers in the order given in table 4 or table 5 , as the case may be, and see to what degree the Munsell dichromatic notation accords with their own perceptions. To the extent that the accord is good, the person will discover how well his type of vision is known; and he may be encouraged to study the basis of the Munsell dichromatic notation, and so gain an additional insight into the relation of his own vision to that possessed by the majority. If the Munsell dichromatic notation fails to accord with his perceptions in ways not to be corrected by viewing the papers through bluish or brownish goggles, it is likely that the observer will find that he has been mistyped by careless administration or interpretation of a routine test, and a retest would be in order. Should the observer find that he is indeed a red-green confusing dichromat, he would have a chance to contribute to the knowledge of color perceptions by studying and reporting the nature and degree of any contradictions.

\section{Analysis of Color-Blindness Tests in Terms of Dichromatic Munsell Notations}

To show how tables 4 and 5 make available the color perceptions of deuteranopes and protanopes, respectively, it will be sufficient to analyse a few of the color-blindness tests based upon lightreflecting objects whose colors are fairly well established.

\section{Holmgren Wool Test}

Probably the oldest color-blindness test still in fairly common use is the famous wool test developed by Holmgren [38]. In this test a considerable number (50 to 200) of small skeins of differently colored yarns are placed in a pile on a flat surface sufficiently spacious and illuminated with full daylight. The skein having the trial color is taken from the pile by the examiner and placed to the side far enough from the others not to be confused with them during the test. The examiner requests the subject to pick out the other skeins that come closest to the test skein in color and to place them beside it. Three tests are recommended by Holmgren, one with a green trial skein, one with a purple or rose colored skein, and a third with a red skein. The color chart (p. 120) "serves to guide the examiner in the choice of colors for the test skeins and in appreciating the mistakes of the deficients. We have tried to render there the colors mentioned in this chapter. We divide them into two classes:

"1. The test colors, that is to say, those which the examiner offers to the subject, and

"2. The confusion colors, that is to say, those which the deficient picks from the pile because he confuses them with that of the specimen."

Table 7 gives the Munsell book notations found in April 1942 for the colors of the chart that forms the frontispiece of a book [38] sent by Holmgren personally to the Smithsonian Institution from which it was turned over on October 10, 1884, to the Library of Congress as volume 150530. Some of the colors were found to be obviously faded; for example color I, supposed to be a green (neither yellow green nor blue green), was found to be a weak greenish yellow, and color IIa, supposed to be a purple, was found to be a light brown. However, the colors relating to the third test seem not to have faded seriously and will serve as an example of how to analyse a color-blindness test by means of dichromatic Munsell notations.

Of the third test Holmgren says, "The red skein is presented to the subject. It should have a vivid red color like the red flag serving as a signal for the railroads. This color corresponds to IIb of the chart, which ought perhaps to incline a little more definitely toward yellowish red. The test, which is to be made only with subjects that are completely defective, ought to be continued until the subject has placed with the test skein all of the skeins having that hue, or until he has selected one or more of the confusion colors (10 to 13). The red-blind (protanope, by present-day terminology) chooses in addition to red some 
shades of green and brown (10 and 11) that for the normal sense appear darker than the red. But the green-blind (deuteranope) chooses contrasting hues which appear lighter than the red."

The analysis of this test consists in finding the dichromatic Munsell notations of the confusion colors (10 to 13 ) and comparing them to the dichromatic Munsell notation of the trial color (IIb). From tables 4 and 5, the dichromatic Munsell notations of these five colors have been obtained by reading the notations for neighboring colors and interpolating among them. For example, color IIb was found to have a Munsell book notation of $5.5 \mathrm{R} 4.8 / 10$. The two closest colors in table 5 are $\mathrm{R} 5 / 10$ and $10 \mathrm{R} 5 / 10$, from which we read protanopic equivalents of $\mathrm{Y} 4.4 / 1.5$ and Y 4.4/4.1, from which in turn by interpolation we would get a protanopic equivalent of $\mathrm{Y} 4.4 / 1.8$ for $5.5 \mathrm{R} 5.0 / 10$, and $\mathrm{Y} 4.2 / 1.8$ for the required book notation 5.5R 4.8/10. This equivalent is given in table 7 , together with deuteranopic and protanopic equivalents similarly found for colors $\mathrm{IIb}, 10,11,12$, and 13 .

TABLE 7. Munsell book notations of the color chart published by Holmgren to indicate how to administer and interpret his wool test; also dichromatic Munsell renotations of five of the colors

\begin{tabular}{|c|c|c|c|c|}
\hline \multicolumn{2}{|l|}{ Holmgren's- } & \multirow{2}{*}{$\begin{array}{l}\text { Munsell book } \\
\text { notation }\end{array}$} & \multicolumn{2}{|c|}{$\begin{array}{l}\text { Dichromatic Munsell } \\
\text { renotation }\end{array}$} \\
\hline Deseription & $\begin{array}{c}\text { Num- } \\
\text { ber }\end{array}$ & & Protanopic & $\begin{array}{l}\text { Deuter- } \\
\text { anopic }\end{array}$ \\
\hline \multirow[t]{3}{*}{ Green ... } & I & $9 \mathrm{Y} \quad 7 / 4$ & & \\
\hline & 1 & $6 \mathrm{Y} 6 \quad / 2$ & & \\
\hline & 2 & $1 \mathrm{Y} 6 \quad / 2.5$ & & \\
\hline \multirow[t]{3}{*}{ Colors confused with green } & 3 & $4 \mathrm{Y} 8 \quad / 5$ & & \\
\hline & 4 & 7 YR $8 \quad / 5$ & & \\
\hline & 5 & 7 YR $7 \quad / 3$ & & \\
\hline Purple & $\mathrm{IIa}$ & $6 \mathrm{YR} 6.5 / 3$ & & \\
\hline \multirow{2}{*}{$\begin{array}{l}\text { Protanopie confusions with } \\
\text { purple. }\end{array}$} & 6 & $5 \mathrm{~PB} 3.0 / 8$ & & \\
\hline & $\{7$ & $5 \mathrm{P} 4.0 / 6.5$ & & \\
\hline \multirow{2}{*}{$\begin{array}{c}\text { Deuteranopic confusions } \\
\text { with purple. }\end{array}$} & 8 & $5 Y 5.5 / 1$ & & \\
\hline & 9 & $7 \mathrm{G} 4.8 / 3$ & & \\
\hline \multirow{3}{*}{$\begin{array}{l}\text { Red } \\
\text { Protanopic confusions with } \\
\text { red. }\end{array}$} & IIth & 5. $5 \mathrm{R} 4.8 / 10$ & Y $4.2 / 1.8$ & Y $4.9 / 3.5$ \\
\hline & 10 & fGY $3.5 / 2$ & Y $3.8 / 1.5$ & Y 3. $7 / 1.3$ \\
\hline & 11 & 10YR 3.6/2 & Y $3.6 / 2.1$ & Y $3.7 / 2.3$ \\
\hline \multirow{2}{*}{$\begin{array}{l}\text { Deuteranopic confusions } \\
\text { with red. }\end{array}$} & 12 & $5 \mathrm{GY} 6.0 / 6$ & Y 6.2/6.2 & Y $6.2 / 5.9$ \\
\hline & 13 & $5 \mathrm{YR} 4.0 / 6$ & Y 4.0/3.6 & Y $4.4 / 4.2$ \\
\hline
\end{tabular}

It is evident from an examination of table 7 why the protanope would be expected to confuse colors 10 and 11 with the red color IIb. The protanopic renotations of these colors differ from that of the red by 0.6 of a value step or less and by only 0.3-chroma step. Similarly, color 13 would be expected to be moderately confusible by deuteranopes with the red color IIb, because the deuteranopic Munsell renotations of these two colors differ by only 0.5 of a value step and 0.7 chroma step; but color 12 should be distinct from the red color IIb to deuteranopes because it is lighter by 1.3 value steps and higher in chroma by 2.4 steps (compare Y $6.2 / 5.9$ with Y 4.9/3.5). This analysis shows that the colors in. Holmgren's book conform to his statements regarding the test with the red skein except for color 12, which was probably not correctly rendered origirally by the lithographer. The other discrepancies are too small to be significant in view of Holmgren's statement: "The resemblance need not be perfect in all respects; there are no two skeins that are exactly alike. The question is particularly on resemblance in hue, and on this account the subject ought to look for those that are similar and belong to the same hue, that which is paler or deeper but of the same color, and so on. . . . Not too much attention should be paid to lightness nor to slight shades of off-gray color."

Similar analyses of tests by means of green and purple skeins give similar corroboration of Holmgren's interpretation of his tests, but these analyses have to be carried out with reference to the actual colors of the green and purple skeins used instead of the faded colors I and IIa of the color chart.

\section{Stilling Pseudo-Isochromatic Plates}

The plates developed by Stilling in 1878 [91] are still used [71,93], and copies and developments of them are widely distributed in this country $[1,41]$. The subject is shown a series of printed pages in succession, each page covered with a pattern of irregularly shaped spots. The spots are so colored and so arranged that numbers can be read on the plates by many observers of normal vision, but to observers having various types of abnormal vision certain of the plates seem to have uniform colors or a uniform mixture of colors, so that no number can be read. Because of this, they are called pseudo-isochromatic plates.

The analysis of a psuedo-isochromatic plate designed to detect protanopia or deuteranopia can be carried out by obtaining the Munsell book notations of typical background spots and typical spots making up the number, reading their pro- 
tanopic and deuteranopic Munsell notations by interpolation in tables 4 and 5, and comparing them. These charts were designed, however, to accord with a view of the color perceptions of dichromatic observers developed by Stilling and described by him in detail by means of a color chart printed in 1909 [92]. It will suffice for illustration of the method to confine our analysis to these printing-ink reproductions of the colors.

Table 8 gives the Munsell book notations of most of the colors found in 1944 on the color chart in the Library of Congress copy of Stilling's paper, Über Entsteheng und Wesen der Anomalien des Farbensinnes [92]. The colors dealing with Stilling's view of the color perceptions of tritanopes have been omitted. Table 8 also shows the protanopic and deuteranopic Munsell renotations corresponding to these book notations read by interpolation from tables 4 and 5 .

From the Munsell book notations of the brown, light brown, and gray colors representing Stilling's view of what the red-green confuser's color perceptions are, it may be seen that his idea of the hue of these perceptions is close to that taken in the present paper; in no case does his estimate depart from the present one $(5 \mathrm{Y})$ by as much as five Munsell hue steps. The value and chroma estimates are also fairly close, particularly those for the color-blind equivalent of blue green; compare $\mathrm{Y} 6.9 / 4.3$ with $\mathrm{Y} 5.8 / 3.6$ and $\mathrm{Y} 6.7 / 3.6$ with $\mathrm{Y}$ $6.0 / 3.8$. The light brown chosen is only slightly darker ( 0.9 of a value step) than that which would represent an average confusion color for protanope and deuteranope. The yellowish gray shown as an estimate of the color-blind perception of violet is very close to the present estimate of the deuteranopic perception of the violet shown (compare Y 5.7/0.6 with Y 6.2/0.9); but since the name given to this sample was gray, the agreement may signify only that there has been a yellowing of the printing-ink representation of gray since its preparation in 1909. Actually the average of the protanopic (PB 5.4/0.4) and deuteranopic (Y $5.7 / 0.6)$ Munsell renotations for the violet shown on the chart is very close to a neutral gray.

The protanopic and deuteranopic confusion

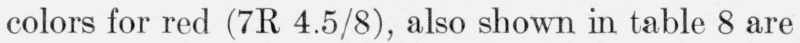
not in very striking agreement; in each case the green is too light and of too high a chroma to be confused by an average protanope or deuteranope. Since the difference between the two colors cor- responds well with the difference between the present estimate of the protanope and deuteranope color perceptions, it is reasonable to suppose that the greenish printing-ink specimens, like the gray, have yellowed with age by one chroma step since 1909. Note that the protanopic equivalent of the red is lower in chroma by $3.4-2.0=1.4$ chroma steps than the deuteranopic equivalent. The green chosen by Stilling as a protanope confusion for red also gives a dichromatic equivalent of lower chroma $(6.0-3.5=2.5,5.8-3.2=2.6)$ than that chosen as the deuteranope confusion for the same red. The two printing-ink specimens thus give the correct idea of the difference between the greens that are confused with red by red-green confusers, but the amount of chroma difference is exaggerated. Similarly, the correct idea of the lightness difference is given $(4.6-4.0=0.6)$, but the amount is in this case too small $(5.6-5.4=0.2$, $5.7-5.4=0.3)$. Stilling was perfectly well aware of the direction of this difference, because he refers to the protanope confusion green as a "somewhat darker green"; whether he had very precise quantitative information cannot be told from this illustrative example because of the uncertainties

TABLE 8. Munsell book notations of the color chart published by Stilling [92] to indicate his view of the color perceptions and confusions of red-green confusers; also protanopic and deuteranopic Munsell renotations of them

\begin{tabular}{|c|c|c|c|}
\hline \multirow{2}{*}{ Stilling's deseription } & \multirow{2}{*}{$\begin{array}{l}\text { Munsell book } \\
\text { notation }\end{array}$} & \multicolumn{2}{|c|}{$\begin{array}{l}\text { Dichromatic Munsell } \\
\text { renotations }\end{array}$} \\
\hline & & Protanopic & $\begin{array}{l}\text { Deuteran- } \\
\text { opic }\end{array}$ \\
\hline Red & $7.4 \mathrm{R} \quad 4.8 / 9$ & Y $4.2 / 2.6$ & Y $4.9 / 4.1$ \\
\hline $\begin{array}{l}\text { Brown seen by color-blind in- } \\
\text { stead. }\end{array}$ & $0.2 \mathrm{Y} \quad 5.9 / 6$ & Y $5.9 / 5.4$ & $\mathrm{Y} 6.2 / 5.8$ \\
\hline Pink & $7.4 \mathrm{R} \quad 6.2 / 8$ & Y $5.9 / 2.1$ & Y $6.4 / 3.1$ \\
\hline Light brown seen instead._._._. & $2.5 \mathrm{Y} \quad 5.9 / 4$ & Y $5.8 / 3.6$ & Y $6.0 / 3.8$ \\
\hline Violet & $9 \mathrm{RP} \quad 5.5 / 6$ & PB 5.4/0.4 & Y $5.7 / 0.6$ \\
\hline $\begin{array}{l}\text { Gray seen by color-blind in- } \\
\text { stead. }\end{array}$ & $7.5 \mathrm{Y} 6.0 / 1$ & Y $6.2 / 0.9$ & Y $6.2 / 0.9$ \\
\hline Blue-green & $2 \mathrm{G} 6.6 / 8$ & Y $6.9 / 4.3$ & $\mathrm{Y} 6.7 / 3.6$ \\
\hline Light brown seen instead...... & $2.5 \mathrm{Y} \quad 5.9 / 4$ & Y $5.8 / 3.6$ & $\mathrm{Y} 6.0 / 3.8$ \\
\hline Yellow-green & $8 \mathrm{GY} \quad 7.3 / 4.6$ & Y $7.5 / 3.7$ & Y 7.4/3.2 \\
\hline $\begin{array}{l}\text { Brown seen by color-blind in- } \\
\text { stead. }\end{array}$ & $0.2 \mathrm{Y} \quad 5.9 / 6$ & Y $5.9 / 5.4$ & $\mathrm{Y} 6.2 / 5.8$ \\
\hline Red ... & 7R $4.5 / 8$ & Y $4.0 / 2.0$ & Y $4.6 / 3.4$ \\
\hline $\begin{array}{l}\text { Yellow-green confused with red } \\
\text { by deuteranope. }\end{array}$ & $3 \mathrm{GY} \quad 5.5 / 6.0$ & Y $5.6 / 6.0$ & Y $5.7 / 5.8$ \\
\hline $\begin{array}{l}\text { Green confused with red by } \\
\text { protanope. }\end{array}$ & $6 \mathrm{GY} \quad 5.3 / 4$ & Y $5.4 / 3.5$ & Y $5.4 / 3.2$ \\
\hline
\end{tabular}


introduced by possible failure of the lithographic process to render the colors correctly, and because of possible changes of the colors with time.

\section{Farnsworth Dichotomous Test (B-20)}

The serial order test devised by Farnsworth [20] at the beginning of the war for screening observers into two groups is particularly easy to analyse in terms of dichromatic Munsell notations, because it is based upon Munsell papers whose color characteristics are known through spectrophotometry $[23,53]$. Table 9 shows the protanopic renotations of these papers arranged in the order given by Farnsworth for a typical protanope; and it also shows the deuteranopic renotations of the papers arranged in the order given by Farnsworth for a typical deuteranope. The values of $W_{p}, w_{p}, W_{d}$, and $w_{d}$ are taken from a previous publication [51].

It will be noted that the arrangement of these papers made by the observer chosen by Farnsworth as a typical protanope is closely, but not perfectly, given by the protanopic Munsell renotation ranging from a yellow of chroma 2.2 through a near neutral (Y 5.2/0.1) to a purple blue of chroma 3.3. A similar correspondence may also be noted between the Farnsworth deuteranopic arrangement and deuteranopic Munsell renotation. Possible causes for the failure of the cor- relation to be perfect are the same as those previously discussed with reference to the chromaticity coordinates, $w_{p}$ and $w_{d}$ [51]. The chief cause of the discrepancies seems to be a slight but consistent difference between the hypothetical average observers defined by eq 1 , on the one hand, and the actual observers chosen by Farnsworth as typical on the other. The discrepancies may thus be taken as indicating about the degree of agreement to be expected between an average red-green confuser and some one observer of the same type chosen at random.

\section{Summary}

A review of the literature on color perceptions mediated by dichromatic, red-green-confusing, visual mechanisms for an observer capable of relating them to normal color perceptions has been carried out, and it shows that both types of such mechanisms (protanopia and deuteranopia) yield color perceptions of two hues, and two hues only, yellow and blue. A review of the chief theories of vision shows that they also all provide for this kind of color perception. A method of deriving protanopic and deuteranopic Munsell notations of colors from their specification in the standard ICI colorimetric coordinate system has

TABLE 9. Protanopic and deuteranopic Munsell renotations of the chips of the Farnsworth dichotomous test [20] arranged in the order found by Farnsworth for a typical protanope and a typical deuteranope, respectively

\begin{tabular}{|c|c|c|c|c|c|c|c|c|c|}
\hline Protanopic arrangement & $\begin{array}{l}\text { Normal } \\
\text { serial } \\
\text { number }\end{array}$ & $0.9733 W_{p}$ & $w_{p}$ & $\begin{array}{l}\text { Protanopic } \\
\text { Munsell } \\
\text { renotation }\end{array}$ & $\begin{array}{l}\text { Deuter- } \\
\text { anopic } \\
\text { arrange- } \\
\text { ment }\end{array}$ & $\begin{array}{c}\text { Normal } \\
\text { serial } \\
\text { number }\end{array}$ & $W_{d}$ & $w_{d}$ & $\begin{array}{l}\text { Deuteranopic } \\
\text { Munsell } \\
\text { renotation }\end{array}$ \\
\hline $1 \ldots$ & 8 & 0.2253 & 0.5628 & Y $5.3 / 2.2$ & 1 & 10 & 0.2143 & 0.5463 & Y $5.2 / 1.8$ \\
\hline $2 \ldots$ & 10 & .2038 & .5405 & Y $5.1 / 1.7$ & 2 & 9 & .2117 & .5412 & Y $5.2 / 1.7$ \\
\hline $3 \ldots$ & 7 & .2096 & .5442 & Y $5.1 / 1.8$ & 3 & 8 & .2292 & .5604 & Y $5.3 / 2.1$ \\
\hline 4.... & 6 & .2061 & .5307 & Y $5.1 / 1.5$ & 4 & 7 & .2097 & .5376 & Y $5.1 / 1.6$ \\
\hline $5 \ldots$ & 9 & .2056 & .5406 & Y $5.1 / 1.7$ & 5 & 11 & .1927 & .5234 & Y $4.9 ! 1.3$ \\
\hline $6 \ldots$ & 11 & .1813 & .5149 & Y $4.8 / 1.0$ & 6 & 12 & .2185 & .5153 & Y $5.2 / 1.2$ \\
\hline $7 \ldots$ & 5 & .1972 & .5011 & Y $5.0 / 0.8$ & 7 & 6 & .2017 & .5185 & Y $5.0 / 1.2$ \\
\hline $8 \ldots$ & 12 & .2034 & .5042 & Y 5. $1 / 0.9$ & 8 & 13 & .1954 & .4891 & Y $5.0 / 0.6$ \\
\hline $9 \ldots \ldots$ & 13 & .1818 & .4779 & Y $4.8 / 0.3$ & 9 & 14 & .2229 & .4761 & Y $5.3 / 0.4$ \\
\hline $10 \ldots$ & 4 & .2199 & .4709 & Y 5. $2 / 0.1$ & 10 & 5 & .1888 & .4835 & Y $4.9 / 0.5$ \\
\hline $11 \ldots$ & 3 & .2162 & .4432 & PB $5.2 / 1.0$ & 11 & 15 & .2138 & .4552 & PB 5.2/0.1 \\
\hline 12 & 15 & .2048 & .4512 & PB 5.1/0.7 & 12 & 16 & .2092 & .4320 & PВ 5.1/1.1 \\
\hline $13 \ldots$ & 14 & .2103 & .4684 & Y 5.1/0.1 & 13 & 4 & .2077 & .4501 & PB $5.1 / 0.3$ \\
\hline $14 \ldots$ & 2 & .2030 & .4242 & PB 5.1/2.0 & 14 & 17 & .1959 & .4101 & PB 5. $0 / 2.0$ \\
\hline $15 \ldots$ & 16 & .2027 & .4309 & PB 5.1/1.6 & 15 & 3 & .2043 & .4227 & PB 5. $1 / 1.6$ \\
\hline $16 \ldots$ & 17 & .1942 & .4145 & PB 5.0/2.4 & 16 & 2 & .1917 & .4037 & PB $4.9 / 2.2$ \\
\hline $17 \ldots$ & 1 & .2321 & .4201 & PB $5.4 / 2.3$ & 17 & 18 & .2118 & .3908 & PB 5. $2 / 3.0$ \\
\hline 18 & 19 & .2071 & .4121 & PB 5.1/2. 5 & 18 & 20 & .2087 & .3891 & PB $5.1 / 3.0$ \\
\hline 19 & 20 & .2164 & .4042 & PB 5. $2 / 3.0$ & 19 & 19 & .2035 & .4013 & PB 5.1/2.5 \\
\hline $20 \ldots \ldots$ & 18 & .2140 & .3998 & PB 5. $2 / 3.3$ & 20 & 1 & .2229 & .4037 & PB $5.3 / 2.5$ \\
\hline
\end{tabular}


been worked out on the basis of this kind of color perception. It is concluded that these notations of colors conform to the usual perceptions of them by protanopic and deuteranopic observers in the sense that the chance of any protanope or deuteranope of average ocular pigmentation having valid ground for objecting to them is remote. These protanopic and deuteranopic Munsell notations therefore serve to relate in a complete and detailed way the color perceptions of red-greenconfusing dichromats with those of normal vision. It is expected that this detailed information will assist in the design of color-blindness tests, and will help color-blind persons to understand the relation of their own visual systems to the normal system and so give them a better chance to avoid the embarrassments and dangers of living in a tridimensional color world with a two-dimensional color detector.

\section{References}

[1] American Optical Co., Pseudo-isochromatic plates for testing color perception (1940).

[2] Tentative definitions of terms relating to paint, varnish, lacquer and related products (ASTM Designation: D $16-42 \mathrm{~T}$ ).

[3] Standard method of test for spectral characteristics and color of objects and materials (ASTM Designation: D 307-44).

[4] J. W. Baird, Color sensitivity of the peripheral retina (Carnegie Institution, Washington, D. C., 1905).

[5] L. H. Bauer and W. MacLake, Military Surgeon 46, 40 (Jan. 1920).

[6] A. Von Baumgartner, Wien Ber. 29, 257 (1858).

[7] O. Becker, Arch. Ophthalmol. [2], 25, 205 (1879); Centr. prakt. Augenheilk. 4, 48 (1879).

[8] Beevor, Trans. Ophthalmol. Soc. U. K. 14, 249 (1894).

[9] W. F. Bonner, J. Am. Med. Assn. 81, 1837 (1923).

[10] W. F. Bonner, Am. J. Ophthalmol. 9, 604 (1926).

[11] J. Brische, Ann. Soc. med. Chir. de Liege 1, 87 (1862).

[12] Committee on colorimetry, Optical Society of America, The Concept of Color, J. Opt. Soc. Am. 33, 552 (1943).

[13] W. Dieter, Z. Sinnesphysiol. 58, 73 (1927).

[14] F. L. Dimmick and M. R. Hubbard, Am. J. Physchol. 52, 242 (1939).

[15] F. C. Donders, Arch. Ophthalmol. 27, (I) 155 (1881); Abstract in Centr. Augenheilk. 5, 403 (1881).

[16] F. C. Donders, Farbengleichungen, duBois-Reymonds Arch. Anat. Physiol. p. 518 (1884).

[17] E. Dreher, Z. Sinnesphysiol. 46, 1 (1912).

[18] F. W. Edridge-Green, Note on a case of asymmetrical colour-blindness, p. 53 (Med. Press, London, 1889); Colour-blindness and colour-perception, 2d ed., p. 197 (1909).
[19] F. W. Edridge-Green, The Hunterian lectures on colour-vision and colour-blindness (Kegan Paul \& Co., 1911).

[20] D. Farnsworth, J. Opt. Soc. Am. 33, 568 (1943).

[21] A. Fick, Zur Theorie der Farbenblindheit, Verhandl. Physik.-Med. zu Wurzburg, N. F. V., p. 129 (1873).

[22] A. Fick, Die Lehre von der Lichtempfindug, in Hermann's Handb. Physiol. III, 139, 207 (Leipzig, 1879).

[23] J. J. Glenn, and J. T. Killian, J. Opt. Soc. Am. 30, 609 (1940).

[24] H. Goldmann, Arch. ges Physiol. (Pfluger's) 194, 509 (1922).

[25] H. Goldmann, Arch. ges. Physiol. (Pfluger's), 210, 70 (1925).

[26] R. H. Goldschmidt, Z. Sinnesphysiol. 50, 192 (1919),

[27] H. Hartridge, Brit. J. Ophthalmol. 4, 318 (1920).

[28] S. P. Hayes, Am. J. Psychol. 22, 369 (1911).

[29] C. A. Hegner, Z. Sinnesphysiol. 49, 18 (1916); Klin. Monatsbl. Augenheilk. 54, 81 (1915).

[30] H. vonHelmholtz, Physiological optics $I I$, 415, translated by J. P. C. Southall (Optical Society of America, Banta, Wisconsin, 1924).

[31] E. Hering, Arch. Ophthalmol. 36, (III) (1890).

[32] G. Hermann, Ein Beitrag zur Casuistik der Farbenblindheit, Inaug. Diss. (Dorpat, 1882).

[33] C. Hess, Arch. Ophthalmol. 35, [4], 1 (1889).

[34] C. Hess, Arch. Ophthalmol. 36, (III) 24 (1890).

[35] R. Hilbert, Arch. ges. Physiol. (Pflüger's) 5\%, 61 (1894).

[36] A. von Hippel, Arch. Ophthalmol. [2] 26, 176 (1880).

[37] A. von Hippel, Arch. Ophthalmol. 27, III, 47 (1881); Abstract in Centr. prakt. Augenheilk. 5, 516 (1881).

[38] F. Holmgren, De la Cécité des Couleurs, dans ses Rapports avec les Chemin-de-fer et la Marine, Authorized translation (Stockholm, 1877).

[39] F. Holmgren, Upsala Läkarfören Förh. 16, 69 (1880).

[40] F. Holmgren, Centr. med. Wiss. Berlin 18, 913 (1880).

[41] F. Holmgren, Upsala Läkarfören. Förh. 16, 145 (1881); brief abstract in Centr. prakt. Augenheilk. 5, 476 (1881). ${ }^{4}$

[42] F. Holmgren, Proc. Roy. Soc. (Liondon) 31, 302 (1881).

[43] F. Holmgren, Upsala Läkarfören. Förh. 16, 308 (1881); brief abstract in Centr. prakt. Augenheilk. 5, 475 (1881).

[44] F. Holmgren, Upsala Läkarfören. Förh. 16, 563 (1881) ; brief abstract in Centr. prakt. Augenheilk. 5, 476 (1881).

[45] F. Holmgren, Upsala Läkarfören. Förh. 16, 222 (1881); brief abstract in Centr. prakt. Augenheilk. 5, 476 (1881).

[46] F. Holmgren, Upsala Läkarfören. Förh. 16, Beil. zu Heft $2 / 3$, p. 1 (1881); brief abstract in Centr. prakt. Augenheilk. 5, 476 (1881).

[47] S. Ishihara, Tests for colour-blindness (Handaya, Tokyo, Hongo Harukicho, 1917).

[48] J. E. Jennings, Am. J. Ophthalmol. 8, 384 (1925).

[49] D. B. Judd and K. L. Kelly, J. Research NBS 23, 355 (1939) RP1239.

[50] D. B. Judd, J. Opt. Soc. Am. 33, 294 (1943). 
[51] D. B. Judd, J. Research NBS 33, 407 (1944) RP1618; J. Opt. Soc. Am. 35, $199 . \quad$ (1945).

[52] H. J. Keegan, J. Opt. Soc. Am. 36, 367 (1946); Ind. Standardization 18, 92 (Apr. 1947).

[53] K. L. Kelly, K. S. Gibson, and D. Nickerson, J. Research NBS 31, 55 (1943) RP1549; J. Opt. Soc. Am. 33, 355 (1943).

[54] A. Kirschmann, Wundt's Philos. Studien 8, 173, 407 (1892-93).

[55] M. Knies, Arch. Augenheilk. 17, 379 (1887); 18, 50 (1887); (Color sensations of the tritanopic observer) 19, 253 (1888)

[56] B. Kolbe, Centr. prakt. Augenheilk. 6, 291-295 (1882).

[57] H. Köllner, Z. Augenheilk. 21, 193, 309 (1909).

[58] H. Kollner, Ueber das Grenzgebiet zwischen normalem Farbensinn und Farbenschwäche, 37th Versammlung J. ophthalmol. ges. zu Heidelberg. p. 245 (1911).

[59] H. Köllner, Die Storeungen des Farbensinnes. (Karger, Berlin, 1912).

[59a] A. König, Ueber "Blaublindheit", Sitz. Akad. Wiss. Berlin, p. 718 (July 8, 1897); Ges. Abhandl., p. 396 (Barth, Leipzig, 1903).

[60] J. von Kries, Die Gesichtsempfindungen und ihre Analyses, duBois-Reymond's Arch. Physiol. Supplement-Band (1882); Die angeborene Farbenblindheit, pp. 134-153, Die erworbene Farbenblindheit, p. 153-157, Die hysterischen und hypnotischen Farbensinn-störungen, pp. 157-158.

[61] J. von Kries, Die Gesichtsempfindungen. Nagel's Handbuch der Physiologie des Menschen. 3, 259 (Braun-schweig, 1905).

[62] J. von Kries, Z. Sinnesphysiol. 50, 137 (1919).

[63] J. von Kries, Note on normal and anomalous colour systems; English Ed. Helmholtz's Treatise on Physiological Optics, 2, 402 (The Optical Society of America, 1924).

[64] C. Ladd-Franklin, Colour-blindness and William Pole, Science 5, 310 (1897).

[65] W. Lohmann, Arch. Augenheilk. 82, 104 (1917).

[66] W. R. Miles and H. Beaumont, Am. J. Ophthalmol. [3], 14, 636 (1931).

[67] W. R. Miles, and H. Craig, Jr., The Personnel J. 9, 437 (1931).

[68] G. E. Müller, Darstellung und Erklärung der verschiedenen Typen der Farbenblindheit nebst Erörterung der Funktion des Stäbchenapparates sowie des Farbensinns der Bienen und der Fische. (Vandenhoeck-Ruprecht, Göttingen, 1924.)

[69] G. E. Müller, Z. Psychol. Ergänzungsb. 17, 18 (1930).

[70] Munsell Book of Color (Library and Pocket ed.), (Munsell Color Co., Baltimore, Md., 1929, 40-huechart edition, 1942).
[71] E. Murray, J. Opt. Soc. Am. 33, 329 (1943).

[72] W. A. Nagel, Z. Psychol. 39, 93 (1905).

[73] W. A. Nagel, Arch. Anat. Physiol. p. 543 (1907).

[74] W. A. Nagel, Z. Psychol. 44, 5 (1910).

[75] E. von Neipperg, Münch. med. Wschr. 79, 1395 (1932).

[76] S. M. Newhall, D. Nickerson, D. B. Judd, J. Opt. Soc. Am. 33, 385 (1943).

[77] Niemetscheck. Ueber Farbenblindheit und Farbensehen, Prag. Vierteljahrsschr. 25, (IV) 224 (1868).

[78] Optical Society of American Committee on Colorimetry, The Concept of Color, J. Opt. Soc. Am. 33, 552 (1943).

[79] J. H. Parsons, An introduction to the study of colour vision, part II. The chief facts of colour blindness, 2d. ed. (University Press, Cambridge, 1924).

[80] H. Piper, Z. Physiol. Sinnesorgane 38, 153 (1905).

[81] F. H. G. Pitt, Characteristics of dichromatic vision, Medical Research Council, Report of the Committee on the Physiology of Vision, XIV; Special Report Series, No. 200 (London, 1935).

[82] W. O. Pole, Proc. Roy. Soc. 8, 172 (1856); Phil. Mag. [4], 13, 282 (1857).

[83] Rayleigh, Nature 25, 64 (1882).

[84] Reichert, Ueber einseitige Farbensinnanomalein, Inaug. Diss. (Freiburg, 1915).

[85] Samojloff, Z. Sinnesphysiol. 41, 367 (1906).

[86] R. W. Shufeldt, Medical Record 23, 319 (Mar. 24, 1883).

[87] Louise L. Sloan and Lorraine Wollach, J. Opt. Soc. Am. 37, 527 (1947).

[88] S. Snell, Peculiar case of colour-blindness, Lancet, p. 727 (Apr. 30, 1881).

[89] S. Snell, Brit. Med. J. No. 1622, p. 222 (1892).

[90] P. Steffan, Arch. ophthalmol. (Graefe's) 27, II, (1881); abstract in Centr. prakt. Augenheilk. 5, 409 (1881).

[91] J. Stilling, Tafeln zur Bestimmung der Röth-Grünblindheit, (Leipzig, 1878).

[92] J. Stilling, Z. Sinnesphysiol. 44, 371 (1909-10).

[93] J. Stilling, Stillings pseudoisochromatische Tafeln zur Prüfung des Farbensinnes, 19th ed. by E. Hertel (Leipzig, Thieme, 1936).

[94] W. Trendelenburg, Klin. Monatsbl. Augenheilk. 10\%, 280 (1941).

[95] A. Tschermak, Licht- und Farbensinn, Bethe's Handbuch der Normalen und pathologischen Physiologie, 12/1. Receptionsorgane 2, 345 (Springer, Berlin, 1929).

[96] H. Voeste, Z. Psychol. 18, 257 (1898).

[97] G. Wilson, Researches on colour-blindness, p. 11 (Sutherland and Knox, Edinburg, 1852).

[98] M. Woienow, Arch. Ophthalmol. 17, II, 241 (1871); Klin. Monatsbl. Augenheilk. 9, 377 (1871).

Washington, May 21, 1948. 